\title{
Investigations of surface related electronic properties in SmB6 and LaAlO3SrTiO3 heterostructures
}

Sanjay Adhikari

Follow this and additional works at: https://researchrepository.wvu.edu/etd

\section{Recommended Citation}

Adhikari, Sanjay, "Investigations of surface related electronic properties in SmB6 and LaAlO3SrTiO3 heterostructures" (2015). Graduate Theses, Dissertations, and Problem Reports. 5030.

https://researchrepository.wvu.edu/etd/5030

This Dissertation is protected by copyright and/or related rights. It has been brought to you by the The Research Repository @ WVU with permission from the rights-holder(s). You are free to use this Dissertation in any way that is permitted by the copyright and related rights legislation that applies to your use. For other uses you must obtain permission from the rights-holder(s) directly, unless additional rights are indicated by a Creative Commons license in the record and/ or on the work itself. This Dissertation has been accepted for inclusion in WVU Graduate Theses, Dissertations, and Problem Reports collection by an authorized administrator of The Research Repository @ WVU.

For more information, please contact researchrepository@mail.wvu.edu. 


\title{
Investigations of surface related electronic properties in $\mathrm{SmB}_{6}$ and $\mathrm{LaAlO}_{3} / \mathrm{SrTiO}_{3}$ heterostructures
}

\author{
Sanjay Adhikari \\ Dissertation submitted \\ to the Eberly College of Arts and Sciences \\ at West Virginia University \\ in partial fulfillment of the requirements for the degree of \\ Doctor of Philosophy in Physics \\ Cheng Cen, Ph.D., Chair \\ Alan D. Bristow, Ph.D. \\ Edward Flagg, Ph.D. \\ Mohindar Seehra, Ph.D. \\ Nianqiang Wu, Ph.D.
}

Department of Physics and Astronomy

Morgantown, West Virginia

2015

Keywords:

Topological Insulators, Kondo Insulators, Oxides

Copyright 2015 Sanjay Adhikari 


\title{
Abstract
}

\section{Investigations of surface related electronic properties in $\mathrm{SmB}_{6}$ and $\mathrm{LaAlO}_{3} / \mathrm{SrTiO}_{3}$ heterostructures}

\author{
Sanjay Adhikari
}

This dissertation reports research performed on two types of two-dimensional systems: $\mathrm{SmB}_{6}$ and $\mathrm{LaAlO}_{3} / \mathrm{SrTiO}_{3}(\mathrm{LAO} / \mathrm{STO})$. $\mathrm{SmB}_{6}$ has been proposed to be a topological Kondo insulator at low temperature. In order to understand carriers/lattice dynamics and their interactions, femtosecond pump-probe spectroscopy is performed in $\mathrm{SmB}_{6}$ single crystals and thin films at variable temperatures. The collective oscillation modes in $\mathrm{GHz}-\mathrm{THz}$ and the change of carrier relaxations is observed as a function of temperature. From the temperature dependent results $f-d$ hybridization, opening of the hybridization gap, "phonon bottleneck", and the possible topological surface state formation is revealed. The topological surface state should support helical Dirac dispersion with momentum-spin lockage. This dissertation reports on current injection in $\mathrm{SmB}_{6}$ thin film with circularly polarized light at oblique incidence. This spin polarized photocurrent is concluded to be a direct result of spin momentum lockage in $\mathrm{SmB}_{6}$.

LAO/STO interface shows 2-dimensional electron gas (2DEG) at the interface when the thickness of LAO is more than 3 unit cell. Carrier properties at the LAO/STO interfaces are highly sensitive to the top surface termination of LAO. The spontaneous dissociation of water on LAO surface is systematically studied by density functional theory and experimental surface characterizations. Extrinsic effects from surface adsorbates were often ignored in the previous studies of the 2DEG. From the experiments, it is found that the dissociated water molecules, especially the surface protons, strongly affect the interface density of states, electron distributions and lattice distortions. The investigations also reveal the importance of additional molecular water layers. These additional water layers, through hydrogen bonds, provide an energetically feasible pathway for manipulating the surface-bonded protons and thus, the interface electrical characteristics. 


\section{Contents}

Dedication ..................................... vii

Acknowledgments ..................... . . ix

List of Figures . . . . . . . . . . . . . . . . . . xiii

List of Tables ...................... . . . . . . . . . . . . .

1 Introduction 1

$1.1 \mathrm{SmB}_{6} \ldots \ldots \ldots \ldots \ldots \ldots \ldots \ldots$

1.1.1 Kondo Insulator . . . . . . . . . . . . . . . . . . . 2

1.1.2 Topological Insulator . . . . . . . . . . . . . . . . . . 4

1.2 Research Problem . . . . . . . . . . . . . . . . . . . . . . 7

$1.3 \mathrm{LAO} / \mathrm{STO} \ldots \ldots \ldots \ldots \ldots$

1.3.1 Theory for Interface Conductivity . . . . . . . . . . . . . . . 12

1.3.2 Research Problem . . . . . . . . . . . . . . . . 14

1.4 Forthcoming Chapters . . . . . . . . . . . . . . 15

2 Ultrafast Laser Cavity 16

2.1 Laser Resonator . . . . . . . . . . . . . . . . . . . . . 17

2.2 Ti:Sapphire Laser . . . . . . . . . . . . . . . . . . . . . . . . . . . . . 21

2.2.1 Mode Locking . . . . . . . . . . . . . . . . . 23

2.2.2 Cavity Design . . . . . . . . . . . . . . . . 27 
2.2 .3 Applications . . . . . . . . . . . . . . . . . . . 31

3 Pump-probe Spectroscopy 33

3.1 Time Resolution . . . . . . . . . . . . . . . . . . . . . 33

3.2 Ultrafast Processes . . . . . . . . . . . . . . . . . . . . . . 34

3.3 Photoinduced Reflectivity Change . . . . . . . . . . . . . . . 36

3.3 .1 Optical Reflectance . . . . . . . . . . . . . . . 37

3.4 Experimental Setup . . . . . . . . . . . . . . . . . . . . . . 39

4 Ultrafast observation of electron hybridization and in-gap states formation in Kondo insulator $\mathrm{SmB}_{6} \quad 41$

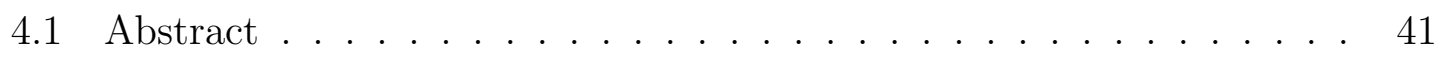

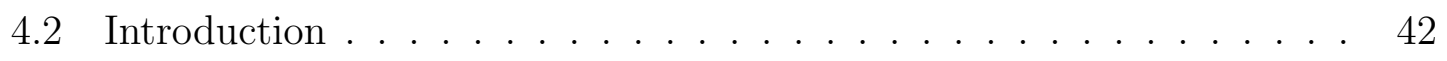

4.3 Experimental Setup . . . . . . . . . . . . . . . . . . . . 44

4.4 Sample Synthesis . . . . . . . . . . . . . . . . . . 44

4.5 Data and Discussion . . . . . . . . . . . . . 46

4.6 Conclusion . . . . . . . . . . . . . . . . . . 57

4.7 Supplementary Information $\ldots \ldots \ldots$

4.7 .1 Transport Measurements . . . . . . . . . . . . . . . . . 59

$4.7 .2 \quad$ Fluence Dependence . . . . . . . . . . . . . . . . . . 61

5 Optical helicity control of surface current in $\mathrm{SmB}_{6} \quad 62$

5.1 Abstract . . . . . . . . . . . . . . . . 62

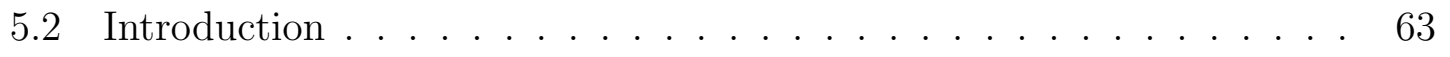

5.3 Experimental Setup . . . . . . . . . . . . . . . . 64

5.4 Results and Discussions . . . . . . . . . . . . 65

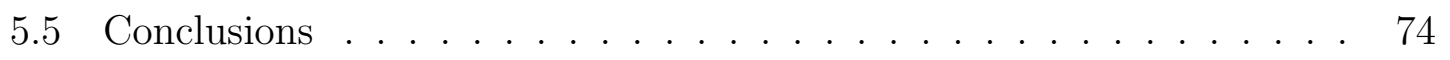


6 Role of surface water adsorptions in $\mathrm{LaAlO}_{3} / \mathrm{SrTiO}_{3}$ interfaces $\quad 76$

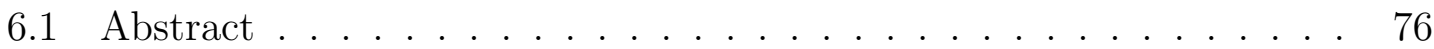

6.2 Introduction . . . . . . . . . . . . . . . 77

6.3 Experimental Methods . . . . . . . . . . . . . . . . . . . 79

6.3 .1 DFT calculations . . . . . . . . . . . . . . . . 79

6.3.2 Heterostructure synthesis . . . . . . . . . . . . . . . . . 80

6.3 .3 X-ray photoemission spectroscopy . . . . . . . . . . . 80

6.3.4 Oxygen plasma surface treatment $\ldots \ldots \ldots \ldots$. . . . 80

6.3.5 C-AFM lithography, low temperature transport and photoconductivity measurements . . . . . . . . . . . . . 81

6.4 Results . . . . . . . . . . . . . . . . . . . . . . . . 82

6.4.1 Water dissociation at the polar surface of $\mathrm{LaAlO}_{3} / \mathrm{SrTiO}_{3}$ het-

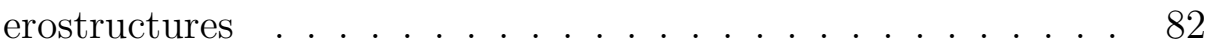

6.4.2 Effects of surface water adsorption on the interface properties 84

6.4.3 Modifying interface properties by oxygen plasma surface treat-

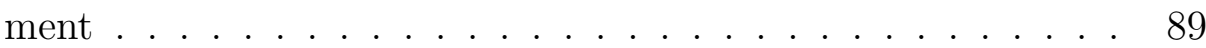

6.4.4 Effects of c-AFM lithography _. . . . . . . . . . . . 90

6.5 Discussion . . . . . . . . . . . . . . . . . . . . . . 93

6.5.1 Effects of charge transfer, electron correlations, and lattice dis-

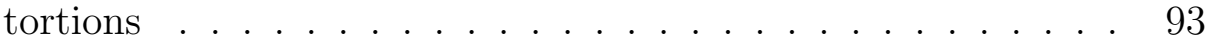

6.5.2 Roles of molecular water layers and the hydrogen bonds . . . . 97

6.5.3 Surface protonation/deprotonation driven by probe biases . . 99

6.5.4 Stability differences between surface bonded $\mathrm{H}$ and $\mathrm{OH}$. . . 101

6.6 Summary . . . . . . . . . . . . . . . . . . . . . . . . 104

$\begin{array}{lll}7 & \text { Summary } & 106\end{array}$ 
$\begin{array}{ll}\text { A Cavity Alignment } & 110\end{array}$

B Troubleshooting of the Laser Oscillator 115

$\begin{array}{ll}\text { C Second Harmonic FROG } & 117\end{array}$

$\begin{array}{ll}\text { D Pump-probe Setup } & 120\end{array}$

D.1 Second Harmonic Generation _. . . . . . . . . . . . . 120

D.1.1 Theory of SHG . . . . . . . . . . . . . . . . 121

D.2 Delay. . . . . . . . . . . . . . . . . . . . . . . . 122

D.3 Modulation . . . . . . . . . . . . . . . . . . . . . 123

D.4 Lock-In Amplifier . . . . . . . . . . . . . . . . . . . . 124

D.5 Detector . . . . . . . . . . . . . . . . . 125

$\begin{array}{ll}\text { E Hall Effect Measurement } & 127\end{array}$

E.1 Hall Bar Configuration . . . . . . . . . . . . . . . . . 127

E.2 Van der Pauw Configuration . . . . . . . . . . . . . . . . . . . 129

F Sample Processing 132

F.1 Photolithography . . . . . . . . . . . . . . . . . . . . . . . 132

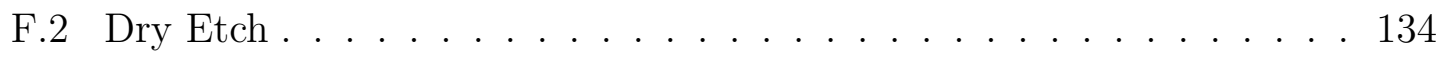

F.3 Metal Deposition . . . . . . . . . . . . . . . . . . . 134

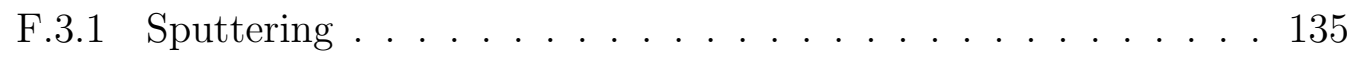

F.3.2 E-beam evaporation . . . . . . . . . . . . . 135

F.4 Oxygen Plasma Cleaning . . . . . . . . . . . . . . . . . . 136

$\begin{array}{ll}\text { G X-ray Photoelectron Spectroscopy } & 137\end{array}$ 
CONTENTS

$\begin{array}{ll}\text { H AFM } & 140\end{array}$

H.1 AC mode . . . . . . . . . . . . . . . . . . . . . . . . . . . 141

H.2 Contact Mode . . . . . . . . . . . . . . . . . . . . . . . . . . . 142

H.3 AFM Lithography. . . . . . . . . . . . . . . . . . . . . . 142

Bibliography 143 


\section{Dedication}

Dedicated to my parents Bodhraj Adhikari and Indrakumari Adhikari. 


\section{Acknowledgments}

I would like to start by thanking my wife. I am indebted forever to my wife Ritu

for her love and support for all these years. Without her support it would have been impossible to finish this dissertation. I would like to thank my parents, who helped me in every step of my life. I would also like to thank my advisor Dr. Cheng Cen. This dissertation would not have been possible without her supervision and guidance. Finally, I would like to thank my dissertation committee, my research collaborators, and the members of our research group. 


\section{List of Figures}

$1.1 f-d$ hybridization in $\mathrm{SmB}_{6} \ldots \ldots \ldots \ldots \ldots$

1.2 Resistance vs. temperature for the $\mathrm{SmB}_{6} \ldots \ldots \ldots \ldots$

1.3 Spin and momentum relationship in a topological insulator . . . . . 6

1.4 Perovskite oxide crystal structure . . . . . . . . . . . . . . . 10

1.5 LAO thickness dependent interface conductivity . . . . . . . . . . 11

1.6 Transport measurements . . . . . . . . . . . . . . . . . . . 11

1.7 Polar catastrophe . . . . . . . . . . . . . . . . . . . . 13

2.1 Essential components of a resonator . . . . . . . . . . . . . 18

2.2 A two-level system . . . . . . . . . . . . . . . . . . . . . 19

2.3 Energy diagram of a three-level laser system. . . . . . . . . . . 20

$2.4 \mathrm{CW}$ and pulsed laser output . . . . . . . . . . . . . . 21

2.5 Energy diagram of a four-level laser system. . . . . . . . . . . . 22

2.6 The absorption and emission spectrum of Ti:Sapphire . . . . . . . 23

2.7 Electric field of a mode-locked laser pulse. . . . . . . . . . . . . 25

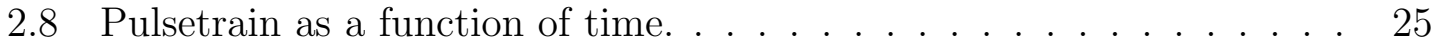

2.9 Kerr lens modelocking . . . . . . . . . . . . . . . 26

2.10 A Ti:Sapphire laser cavity . . . . . . . . . . . . . 27

2.11 Spectrum . . . . . . . . . . . . . . . 30 


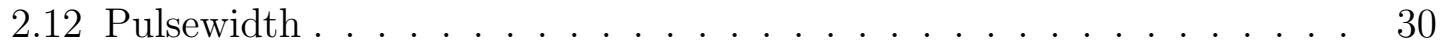

2.13 Pulsetrain. . . . . . . . . . . . . . . . . . . . . 31

3.1 Cartoons showing light matter interactions . . . . . . . . . . . 35

3.2 Time scales of some of the ultrafast phenomenons . . . . . . . . 36

3.3 Ultrafast reflectivity signal . . . . . . . . . . . . . . . . . . . 37

3.4 Pump-probe spectroscopy setup . . . . . . . . . . . . . . . . . 39

4.1 Temperature dependence of the time-resolved PI reflectivity measured in $\mathrm{SmB}_{6} \ldots \ldots \ldots \ldots \ldots \ldots \ldots \ldots$

4.2 Temperature dependence of $f$-band plasmon mode . . . . . . . . 47

4.3 Phonons in $\mathrm{SmB}_{6}$ acquired from neutron scattering . . . . . . . . 48

$4.4 f$-band plasmon mode in thin film . . . . . . . . . . . . . 50

4.5 Temperature dependence of the thermal carrier densities $n(T) \ldots . \quad 52$

4.6 Strain wave in thin film sample . . . . . . . . . . . . . 55

4.7 Transport measurements . . . . . . . . . . . . . . . . . . . . 59

4.8 Conductivity fit $\ldots \ldots \ldots \ldots \ldots \ldots \ldots$

4.9 Pump induced reflectivity as a function of the pump fluence . . . . 61

5.1 Transport properties of $\mathrm{SmB}_{6}$ thin films $\ldots \ldots \ldots \ldots$

5.2 Photovoltage (PV) generated by linearly polarized light . . . . . . . 66

5.3 Circular photogalvanic effects (CPGE) in $\mathrm{SmB}_{6}$ thin film . . . . . 68

5.4 Suppression of CPGE voltage in $20 \mathrm{~nm}$ films $\ldots \ldots \ldots \ldots . \ldots 72$

5.5 Temperature dependences of $\mathrm{PVs}$ in $\mathrm{SmB}_{6}$ thin film . . . . . . . 74

6.1 Water dissociation at $\mathrm{AlO}_{2}$ terminated $\mathrm{LaAlO}_{3}$ surface. . . . . . . . 82

6.2 Layer resolved electronic density of states (DOS) in 3 uc LAO/STO heterostructure for surface absorptions . . . . . . . . . . 84 
6.3 Layer resolved electronic density of states (DOS) in 5 uc LAO/STO heterostructure for surface absorptions $\ldots \ldots \ldots \ldots$. . . . 85

6.4 Total densities of conduction band states below Fermi level . . . . . . 86

6.5 Simulated lattice structures of $3 \mathrm{uc} \mathrm{LaAlO}_{3} / \mathrm{SrTiO}_{3}$ heterostructure . 87

6.6 Total densities of conduction band states below Fermi level (c) and lattice distortions . . . . . . . . . . . . . . . . . 88

6.7 Suppression of the interface conductance induced by oxygen plasma

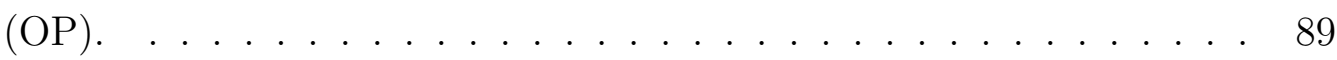

6.8 Roles of oleic acid molecules bonded to the top surface . . . . . . . 90

6.9 Biased AFM probe induced proton hopping . . . . . . . . . . . 97

6.10 Retentions of the LAO surface O-H bonds in different environments. . 103

A.1 A schematic for pump alignment . . . . . . . . . . . . . 111

A.2 A schematic for alignment of one arm . . . . . . . . . . . . . 112

A.3 A schematic for cavity alignment . . . . . . . . . . . . . . . 113

C.1 A SH-FROG setup. . . . . . . . . . . . . . . . . . . 118

C.2 Schematic diagram of a FROG algorithm. . . . . . . . . . 119

D.1 SHG by a BBO crystal . . . . . . . . . . . . . . . . 120

D.2 Energy-level diagram describing SGH . . . . . . . . . . . . . 122

D.3 Translation stage . . . . . . . . . . . . . . . . . . . . 123

D.4 Photoelastic modulator schematic . . . . . . . . . . . . . . 124

D.5 Schematic diagram of a lock-in amplifier. . . . . . . . . . 125

D.6 Feedback loop in an autobalance detector . . . . . . . . . . . . 126

E.1 A Hall bar configuration for Hall effect measurement. . . . . . . . . 128

E.2 A van der Pauw configuration for measuring sheet resistance . . . . 130 
E.3 A van der Pauw configuration for Hall measurement . . . . . . . . . . 131

F.1 Photolithography steps . . . . . . . . . . . . . . . . 133

F.2 Dry etching . . . . . . . . . . . . . . . . . 134

F.3 Metal contacts deposition . . . . . . . . . . . . . . 135

G.1 A working mechanism of XPS. . . . . . . . . . . . . . . 138

G.2 XPS spectra of Sm $4 d$ spectrum in $\mathrm{SmB}_{6} \ldots \ldots$. . . . . . . . 139

H.1 A circuit diagram for AFM. . . . . . . . . . . . . . . . . . 141 


\section{List of Tables}

2.1 Laser cavity parameters . . . . . . . . . . . . . . . . 28

2.2 Estimation of GDD by the optics inside of the laser cavity. . . . . . . 29 


\section{Chapter 1}

\section{Introduction}

Two-dimensional (2D hereafter) systems have very interesting properties when compared to the bulk. Electrons in these systems are confined to move in the plane. Usually, 2D systems are crystalline materials which consists of single layer thick atoms. Some examples of these materials are graphene, germanene, silicene, etc. However, there are other materials that show 2D properties without being a single atom thick. These materials can show 2D properties either at the atomically flat heterointerface or on the top surface of a material. $\mathrm{LaAlO}_{3}$ grown on top of $\mathrm{TiO}_{2}$ terminated $\mathrm{SrTiO}_{3}(\mathrm{LAO} / \mathrm{STO})$ has $2 \mathrm{D}$ electron gas (2DEG) or sometimes also called 2D electron liquid at the interface, when the thickness of the LAO is more than 3 unit cell [1]. $\mathrm{SmB}_{6}$ is another material with potential 2D topological surface state at low temperatures [2]. These materials are introduced below in detail.

\section{$1.1 \quad \mathrm{SmB}_{6}$}

In 1969, the transformation of $\mathrm{SmB}_{6}$ from metallic to semiconducting state, with the lowering of the temperature, was first reported [3]. Back then, $\mathrm{SmB}_{6}$ was clas- 
sified as mixed valence compound. The transformation from metallic to semiconducting state was associated with the changing of electronic configuration of Sm ion from trivalent to divalent with the decrease in temperature. However, by 1992, the material was re-classified as a Kondo insulator [4]. Details on "Kondo" nature is given in the next paragraph.

\subsubsection{Kondo Insulator}

"Kondo effect" is a phenomenon (named after Jun Kondo) which describes the scattering of conduction electrons in a metal from magnetic impurities which results in a characteristic change in electrical resistivity with the change in temperature [5]. Materials with strong electron correlations often exhibit exotic ground states such as the heavy fermion behavior, Mott or Kondo insulation and unconventional superconductivity. $\mathrm{SmB}_{6}$ is one one of those system where there is strong electron electron correlation. The band structure of $\mathrm{SmB}_{6}$ consists of a flat band of $4 f$ electrons and an almost free quadratic $5 d$-band. At high temperatures (close to room temperatures), the two bands only weakly interact. At low temperatures, the periodic arrangement of spins created a Kondo lattice and the hybridization of localized $f$-electrons with conduction band ( $d$-band) electrons occurs due to which a small bandgap opens up (see Figure 1.1) [6]. 


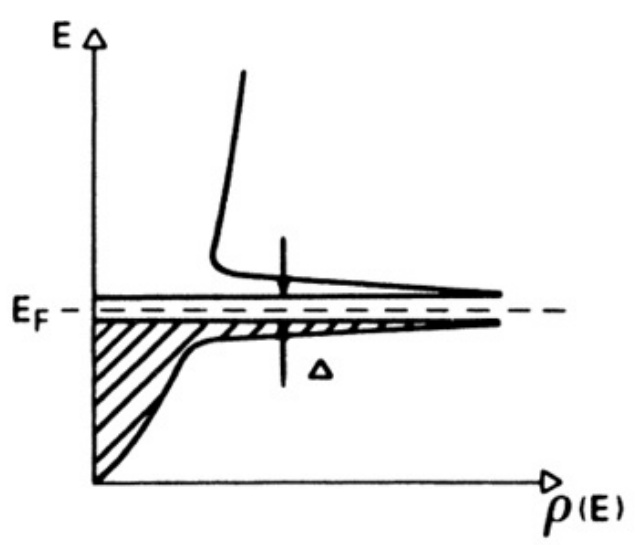

Figure 1.1: A cartoon showing the $f-d$ hybridization in $\mathrm{SmB}_{6}[7]$ leading to gap formation with the width of the gap given by $\Delta\left(E_{g}\right.$ and $\Delta$ are used interchangeably throughout the text). In this figure, $\mathrm{E}_{F}$ is the Fermi level and $\rho(\mathrm{E})$ is the density of state.

In a Kondo system, after the formation of the gap, if the chemical potential lies outside the gap $(\Delta)$ it is called heavy fermion metal. If the chemical potential lines within the gap, it is called a Kondo insulator. The hybridization produces many interesting properties such as: insulating at low temperature, and highly conducting at room temperature. Furthermore, conducting surface state has been reported in this material at very low temperature from transport measurements [2]. In the figure 1.2 , the saturation of resistance at low temperature, unlike most insulators where the resistance continues to rise, is attributed to the conducting surface state. This conducting surface state is predicted to be a topological gapless surface state due to strong spin-orbit coupling in Kondo insulators [8]. Even after the formation of surface state, the bulk of the material continues to be insulating $[9,10]$. This property of $\mathrm{SmB}_{6}$ where bulk is insulating but the surface is still conducting has generated tremendous interest (surface conductance on a insulator is a signature feature of topological insulator). More information on the possible topological nature of $\mathrm{SmB}_{6}$ is provided on the next few paragraphs. 


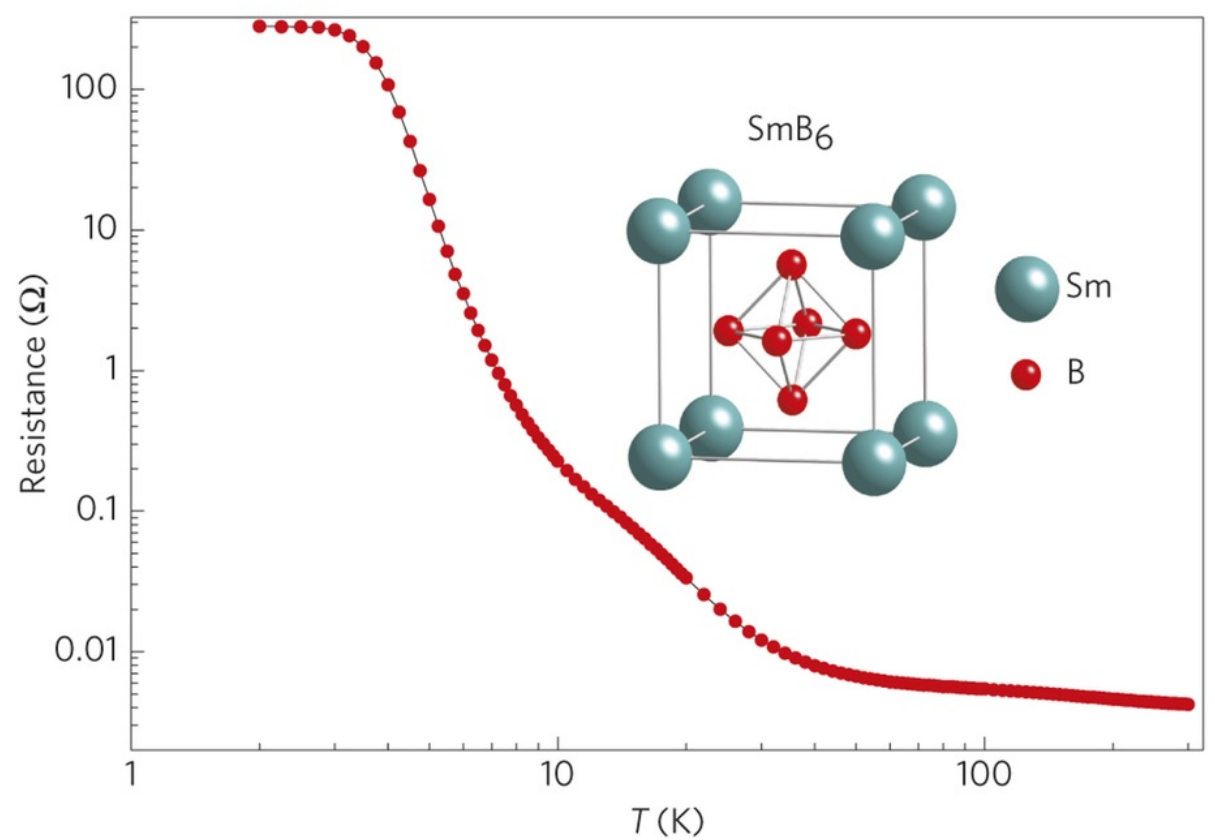

Figure 1.2: Resistance vs. temperature for the $\mathrm{SmB}_{6}$ [2]. Inset to the figure shows the crystal structure is cubic. The low resistance at temperature close to room temperature means the material is highly metallic. The increase in resistance with decrease in temperature is associated with the band gap $\Delta$ formation in this material. The saturation of resistance at very low temperature is attributed to surface state formation.

\subsubsection{Topological Insulator}

In order to classify a material as a topological insulator (TI), it must satisfy the following conditions:

- The material should have electrical conduction on the surface due to a special surface electronic states. Figure 1.3 (b) shows the 2D electronic state on the surface of a 3D TI. The surface state in a TI is a 2DEG. However, the bulk of the material is still insulating with some finite band gap. Hamiltonian of a 
surface state can be represented by:

$$
H_{\text {surf }}=\hbar v_{\|}\left(k_{y} \sigma_{x}-k_{x} \sigma_{y}\right)
$$

In equation above, $v_{\|}$is the Fermi velocity, $k_{x}$ and $k_{y}$ are momentum in $x$ and $y$ axis, and $\sigma_{x}$ and $\sigma_{y}$ are Pauli matrices. From the equation above, it is clear that there is a linear relationship between energy and momentum in the surface state.

- On the topological surface, the spin and the momentum are locked perpendicular. In figure 1.3 (a), the spin and the electron momentum are perpendicular to one another. Spin and momentum being perpendicular can be seen in the Hamiltonian. The surface of a TI supports electron motion in any direction along the surface. The momentum of electron determines the spin direction and vice versa.

- The surface state should have time reversal symmetry. Mathematically, time reversal symmetry can be written as:

$$
E(k, \uparrow)=E(-k, \downarrow)
$$

In above equation, $\uparrow$ and $\downarrow$ represents spin up and spin down of the electrons. Dirac cone band structure found in a TI (see figure 1.3) satisfies the time reversal condition.

- Furthermore, the outcome of this time reversal symmetry is topological protection in the surface state. Topological protection means the carriers in the surface state cannot be scattered back by impurities or imperfections [11]. 


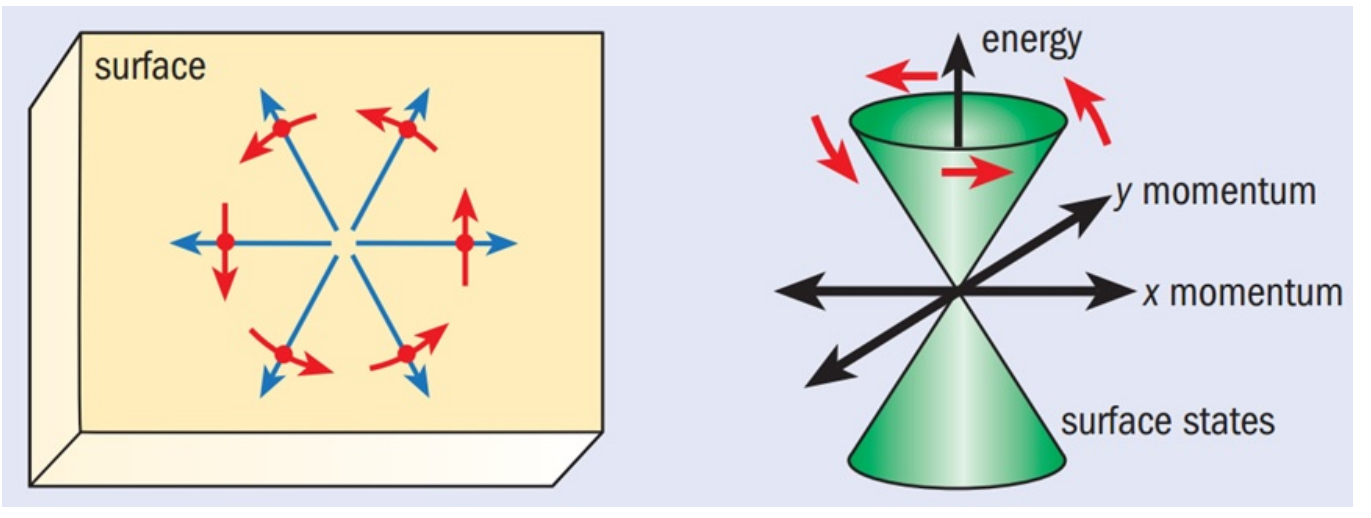

Figure 1.3: Spin and momentum relationship in a topological insulator [11].(a) Surface state of a TI showing momentum and spin being perpendicular. Spin direction is shown with red arrow and momentum direction is shown with blue arrow. (b)The 2D energy-momentum diagram of a TI with "Dirac cone".

Recent theories on topological Kondo insulator (TKI) $[8,12-21]$ have predicted that $\mathrm{SmB}_{6}$ can have topological non-trivial surface state with odd number of Dirac surface bands. The hybridization and odd parity wavefunction can lead to strong spin-orbit coupling and give rise to a topological surface state that dominates electron conduction at low temperatures. First principle calculations [13-16, 18-20] have proposed that the surface state has three Dirac bands at $\Gamma$ and $X / Y$ points in the (100) surface.

\section{Applications for TI}

One of the challenge in the field of spintronics is the generation and control of spin current. In most materials, spin current cannot be generated easily. However, as discussed earlier, topological surface has electron spin perpendicular to the momentum. As a result, the the orientation of spin can be precisely controlled by controlling the direction of current. Hence, TIs might be the material of choice for the further development in the field of spintronics.

When a true topological insulator and a superconductor are brought into a close 
contact, the topological surface can be made superconducting. Superconducting topological surface state has been predicted to support Majorana fermions [22]. Majorana fermion is one of the mysterious fundamental particles in nature that is its own antiparticle [23]. So, when two Majorana fermions come in contact they annihilate one another. The creation of Majorana fermions using topological insulator would be a significant breakthrough in physics, since it has not been discovered yet.

\subsection{Research Problem}

In the last few years, tremendous experimental progress has been made on $\mathrm{SmB}_{6}$. On top of transport measurement revealing the existence of surface layer $[2,9,10,24$, 25] (see Figure 1.2), other measurements such as: point-contact spectroscopy [26], scanning tunneling spectroscopy (STS) [27,28], angle resolved photoemission spectroscopy (ARPES) [26, 29, 29-32] and magnetometry [33] have provided further evidences of the surface state (SS). Sensitivity to magnetic dopants, Dirac-like dispersion and spin helicity have been discovered $[2,26,29,33]$ in support of the TKI theory. However, there is no consensus whether the SS is a topological surface state or regular SS. Nontopological SSs associated with the Boron dangling bonds, common in hexaborides $[34,35]$, generates additional complexities to the interpretation. Evidences of surface Boron suboxide formation [36], chemical potential shift at polar and non-polar surfaces [37], and polarity driven surface metallicity [38] have been found in $\mathrm{SmB}_{6}$. Metallic bulk states [39] and impurity states based hopping mechanism [40] are suggested as alternative contributors to the low temperature resistance plateau. The entanglement of surface state topology with strongly correlated bulk states and complicated surface chemistry makes $\mathrm{SmB}_{6}$ a highly attractive system for more in-depth investigations. 
Most of the measurements mentioned above address equilibrium states, where material properties are often described by effective thermodynamic parameters. On the contrary, pump-probe technique abruptly disturbs the material equilibrium by laser pulses and studies the ultrafast re-equilibrium process as a function of time. By examining various characteristic dynamics in time domain, this technique can help distinguish many intrinsic degrees of freedom that are entangled at equilibrium. Examples include the study of electron-phonon interaction [41-44], order parameter fluctuation [45], pseudogap formation [46], and spin-orbit coupling [47] in many strongly correlated materials. In $\mathrm{SmB}_{6}$, besides the pioneering work by Demsar et al. [42], recent works of time resolved Terahertz spectroscopy [48-50] and pump-probe photoemission spectroscopy [51] have provided valuable insights into the low temperature band structure and the surface band bending. In this dissertation, photoexcited electron dynamics in $\mathrm{SmB}_{6}$ are investigated by optical pump-probe technique at different temperatures. Observations of the characteristic $f$-electrons plasmon help pin-point the hybridization gap opening temperature. Probing the photocarrier relaxation in time domain enables the quantification of the gap and sensitive detection of the possible in-gap states at much lower temperature. To evaluate the robustness of the observed properties, experiments are performed on two types of samples with distinct crystalline characters: single crystal and polycrystalline (as grown) thin film.

One of the outcome of a topological surface state is Dirac-like dispersion with spin helicity. Recently in $\mathrm{Bi}_{2} \mathrm{Se}_{3}$, another TI, spin polarized photocurrent dependence on the helicity of incident light has been theorized and experimentally reported [52, 53]. Since photons in Circularly Polarized (CP) light have a well-defined angular momentum, CP light can couple to the spin of the surface electrons in surfaces with broken rotational symmetry. Experimentally, the rotational symmetry of a surface 
can be broken by an obliquely incidental (OI) light. Since the SS of a TI has spinmomentum-locking, the coupling of light can result in dc transport that is sensitive to the helicity (right versus left-circular polarization) of the incident light. However, in $\mathrm{SmB}_{6}$, no direct spin polarized photocurrent has been reported so far. In this dissertation, we report that the illumination of $\mathrm{SmB}_{6}$ with $\mathrm{CP}$ OI light generates a photovoltage (PV) and reversal of the CP light switches the polarity of the PV. To explain the result, we propose that the change in direction of PV with the change in the helicity of the light, also called circular photogalvonic effect (CPGE), is due to the topological helical Dirac fermions on the surface of the $\mathrm{SmB}_{6}$ and can not be due to Rashba type spin-orbit coupling of the surface as a result of band bending or dangling bonds common in this system.

\section{$1.3 \quad \mathrm{LAO} / \mathrm{STO}$}

LAO and STO are materials that falls under the category of perovskite oxide. Perovskite oxides have distinctive structure with general formula of $\mathrm{ABO}_{3}$. Figure 1.4 shows a unit cell (uc) of a perovskite oxide. 'A' site ions located on the corners of the lattice are usually alkaline earth or rare earth elements. 'B' site ions located on the center of the lattice are usually transition metal elements and the face center site are occupied by oxygen. 


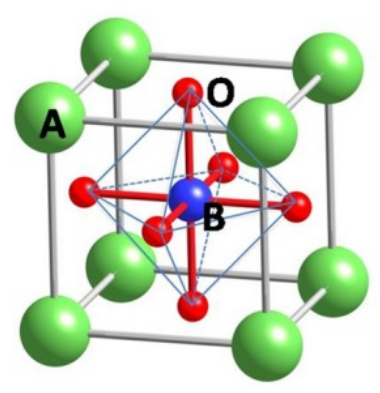

Figure 1.4: A cartoon showing the perovskite oxide crystal structure [54].

$\mathrm{LAO}$ and STO are band insulator. The bandgap of LAO is $5.6 \mathrm{eV}$ and the bandgap of STO is $3.2 \mathrm{eV}$ [55]. In addition to interface conductivity, the interface shows many other interesting properties such as: the tunability of the 2DEG with electric field [56], magnetism at the interface [57], and even superconductivity [58]. Figure 1.5 shows the dependence of interface conductivity as a function of LAO thickness. In LAO/STO sheet conductance $\left(\sigma_{S}\right)$ increases by orders of magnitude when the thickness (d) of the LAO is more than 3 uc. Similarly, the sheet carrier density $\left(n_{S}\right)$ increases by tens of order of magnitude when the thickness of LAO is more than 3 uc. The increase of conductivity and carrier density clearly means that the interface transitions into metallic when the LAO thickness is increased from 3 uc. 

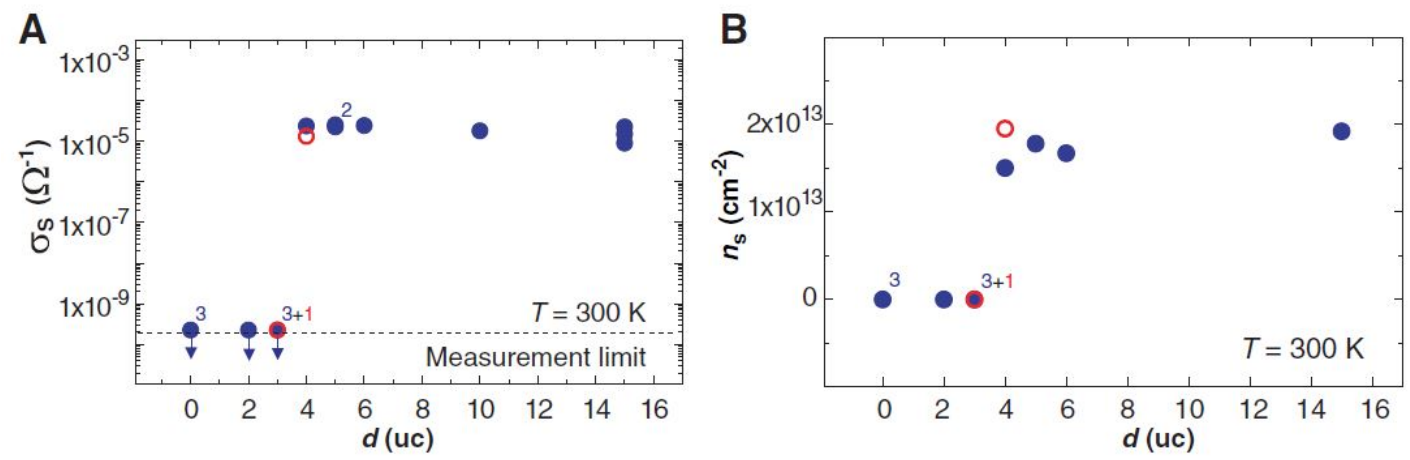

Figure 1.5: Dependence of LAO/STO interface conductivity on the LAO thickness [56].(A) Sheet conductance and (B) carrier density of the interface plotted as a function of the number of LAO unit cells.

Resistance measurements as a function of temperatures (figure 1.6) not only shows the metallic nature (inset to the figure) of the interface but also superconductivity. At very low temperature, the resistance drops from $\mathrm{k} \Omega$ range to zero for both $8 \mathrm{uc}$ and $15 \mathrm{uc}$ (of LAO thickness) sample.

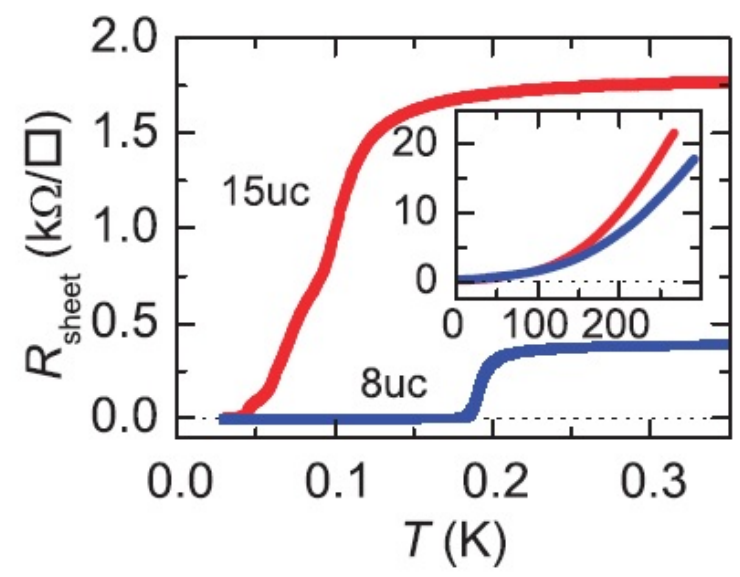

Figure 1.6: Transport measurements of the LAO/STO interface as a function of temperature [58]. Dependence of the sheet resistance on $T$ of the 8-uc and 15-uc samples. Inset: sheet resistance versus temperature measured between $4 \mathrm{~K}$ and 300 $\mathrm{K}$.

This interface conductivity, that can be tuned with local electric field, has led 
to many potential applications such as on demand high-electron mobility transistors $[59,60]$ and photodetectors [61]. Nonetheless, the studies on the physical origin of 2DEG at the interface have been inconclusive. There are multiple theories such as: polar catastrophe [62-64], accumulation of carriers [55], and oxygen vacancies in STO [65] to explain the origin.

\subsubsection{Theory for Interface Conductivity}

As explained in the previous paragraph, a complete theory for the formation of 2DEG has not been postulated yet. However, "polar catastrophe" leading to electronic reconstruction is one of the most accepted model for explaining the origin of interface conductivity. This is the theory used for explaining the results in this dissertation. $\mathrm{LAO}$ is a polar material with alternating layer of $\mathrm{LaO}^{+}$and $\mathrm{AlO}_{2}^{-}$. The alternating layers of charges in LAO give rise to a non-zero electric polarization perpendicular to the interface. On the other hand, STO is a non-polar layered material with $\mathrm{SrO}$ and $\mathrm{TiO}_{2}$ as two alternating layers. The discontinuity at the interface induces an electric potential in LAO film that diverges with the film thickness. This unstable scenario is called "polar catastrophe". This non-reconstructed atomically sharp interface is energetically costly to maintain. Hence, the system has to balance the polar discontinuity and minimize the potential energy through electronic reconstruction. Electronic reconstruction leads to a net charge transfer of $1 / 2$ electron per unit cell from the top LAO surface to the top STO layer as shown in figure 1.7 (c) and (d). This redistribution of charge forms 2DEG at the heterointerface. 

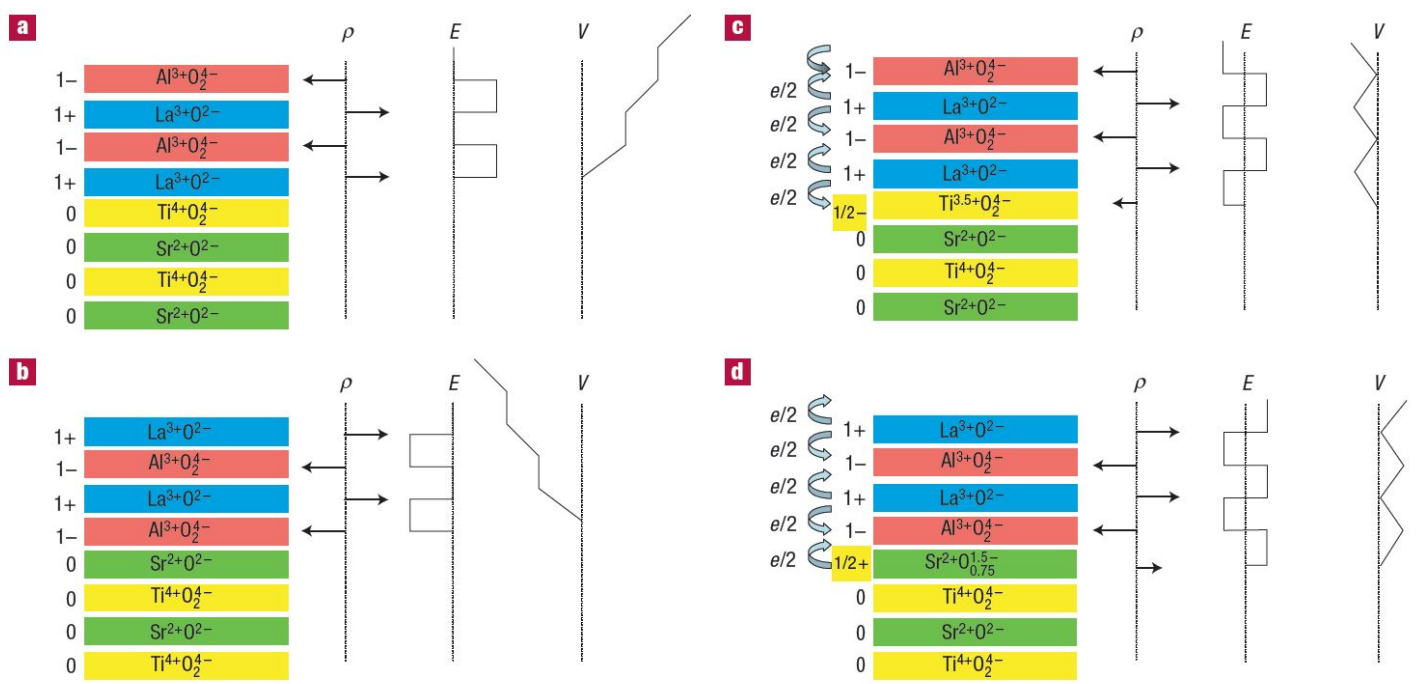

Figure 1.7: Charge distribution before and after the polar catastrophe at the interface of LAO/STO with different STO top surface termination [64]. (a) Non-reconstructed $\mathrm{LaO} / \mathrm{TiO}_{2}$ interface. A positive potential exists in LAO layer and diverges with film thickness. (b) A similar but negative potential occurs in the case of $\mathrm{AlO}_{2} / \mathrm{SrO}$ interface. (c) At $\mathrm{LaO} / \mathrm{TiO}_{2}$ interface, adding $1 / 2$ electron per unit cell to top $\mathrm{TiO}_{2}$ layer can minimize the potential in LAO. (d) At $\mathrm{AlO}_{2} / \mathrm{SrO}$ interface, adding 1/2 hole per unit cell to top SrO layer can minimize the potential in LAO.

This model predicts that both $\mathrm{TiO}_{2}$ and SrO terminated STO should have interface conductivity because both systems should have "polar catastrophe" situation. For $\mathrm{TiO}_{2}$ terminated STO, the interface should be electron doped and for SrO terminated STO the interface should be holes doped. However, experimentally it has been found that only $\mathrm{TiO}_{2}$ terminated STO has interface conductivity while $\mathrm{SrO}$ terminated STO has as insulating interface [1]. This discrepancy could be explained by the fact that Ti has 2 valance states $(3+$ and $4+)$ and $\mathrm{Sr}$ does not. Therefore, Ti can take extra electron to avoid the diverging potential while Sr cannot. 


\subsubsection{Research Problem}

In "polar-nonpolar" oxide heterostructures of LAO/STO, the formation of interfacial 2DEG has generated widespread interest [66]. As explained earlier, a mechanism involving the charge transfer between the surface and the interface ("polar catastrophe") was proposed to explain the formation of interfacial 2DEG. Furthermore, carrier depletion due to oxygen dangling bonds at the surface have been suggested for the interface conductivity [67]. Different capping layers have been also explored for tuning of the 2DEG [68-70]. Furthermore, effects of liquid water on the interface

electrical properties have been experimentally observed $[71,72]$. However, the roles of dissociated water molecules have not been systematically studied. In addition, reversible interface metal-insulator transition controlled by conducting atomic force microscope (c-AFM) were previously reported $[59,73]$. Experiments performed in controlled environments have linked the effects directly to the surface water [74].

Despite these interesting experimental findings, several critical questions remain unanswered: What are the energetically favorable water absorption structures? How do they alter the local band structure? How do the adsorbed water respond to the external field or environmental change? This dissertation seeks to answer these specific questions through first-principle modeling and tailored experiments. Results from the experiment and the corresponding model shows that a partially dissociated water layer is found to strongly bond to the polar LAO surface. Further, dissociated protons, hydroxyl ions and chemisorbed water molecules exhibit distinct effects on the interface density of states. Also important is the thermodynamics of the surface proton migration bridged by physically absorbed water molecules. The multi-faceted role of water is undoubtedly critical in shaping the unique characters at LAO/STO interfaces. Therefore, the results help in understanding of the surface related effects 
which can be vital in quality control [75-79] and material engineering [76, 80-85].

\subsection{Forthcoming Chapters}

In order to study the materials introduced in this chapter a femtosecond laser cavity system was built which then was used for ultrafast spectroscopy. The next two chapters (2 and 3 ) will be on laser cavity followed by the pump-probe spectroscopy. Chapter 4 and 5 are dedicated to the experiments on $\mathrm{SmB}_{6}$ while chapter 6 is on LAO/STO. These three chapters are self contained, meaning they each will have results, discussions, conclusions, and future directions. These chapters are based on the manuscripts submitted or to be submitted for the publication. The chapter 7 summarizes the results of the experiments on $\mathrm{SmB}_{6}$ and LAO/STO. The concluding chapter is followed by appendices which will help in understanding the experimental details. 


\section{Chapter 2}

\section{Ultrafast Laser Cavity}

The term "laser" is an acronym for (L)ight (A)mplification by (S)timulated (E)mission of (R)adiation. The basic oprerating principles of the laser were introduced by Charles Townes and Arthur Schalow from the Bell Telephone Laboratories in 1958 [86]. In order to understand the physics of a laser, it is essential to understand the meaning of each terms. "Light" is a type of an electromagnetic radiation. In order to understand the other terms in "laser", one needs to understand concept of energy levels. The atomic model proposed by Neils Bohr in 1913 [87] is a simple model to explain the concept of energy levels ${ }^{1}$. In this model, electrons in an atom orbit around the nucleus. The electrons can only orbit stably, without radiating, in certain discrete orbits. These orbits are associated with definite energies and can also be called energy levels. In these orbits, the electron's are free to move without losing energy. Electrons can gain energy by jumping from lower energy orbit to a higher energy orbit. Electron can lose energy by jumping from higher energy orbit to a lower energy orbit. Electrons absorb or emit electromagnetic radiation with a frequency $(\nu)$ determined by the energy difference of the orbits; according to the

\footnotetext{
${ }^{1}$ The simple Bohr model, a basis for quantum mechanics, is not a complete model.
} 
Planck's relation [88]:

$$
E_{1}-E_{2}=\Delta E=h \nu
$$

Where, $h$ is the Planck's constant, $E_{1}$ and $E_{2}$ are the energy of the two discrete orbits, and $\Delta E$ is the energy difference. Frequency and the wavelength $(\lambda)$ are related by the equation 2.2 :

$$
\lambda \nu=c
$$

where $c$ is the speed of light. Now, the equation 2.1 can be rewritten as

$$
\Delta E=\frac{h c}{\lambda}
$$

From equation 2.3, it is clear that an electron can radiate a specific wavelength of light (a photon) that equals the gap energy. This discussion on the Bohr model explains the (E)mmision of (R)adiation in a "laser".

\section{$2.1 \quad$ Laser Resonator}

The (A)mplification by (S)timulated (E)mission can be explained by describing the working principle of a laser resonator. A simple laser consists of a gain medium, a high reflecting mirror (HR), an output coupler (OC) which is partially transmissive, and an energy source for pumping. Pumping could be either done using electrical current or another light source. Pumping using electrical current is called electrical pumping. Pumping using a light source is called optical pumping. The essential components of a laser are shown in figure 2.1. 


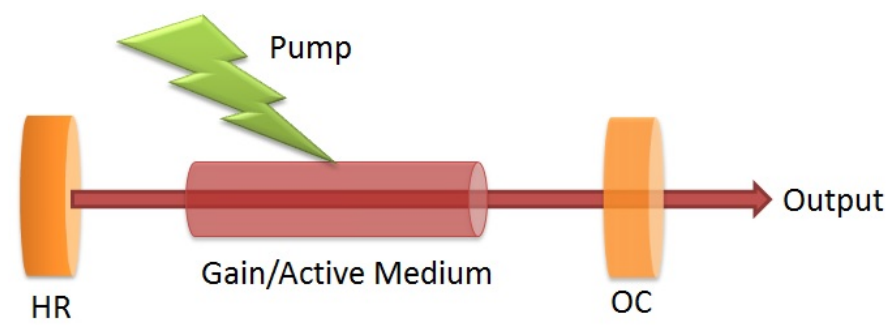

Figure 2.1: Essential components of a resonator

In order to explain the amplification of light, first, a simple two-level system which consists of a ground state and an excited state will be explained ${ }^{2}$. When an electron is excited from ground level to a higher energy level, it wants to decay back 3. It is energetically favorable for an electron to stay in a lower energy level. The decaying electron can emit a photon with energy equivalent to the energy gap. This emission is called "spontaneous emission" (see figure 2.2). A two-level system can also have "induced emission". However, there is no amplification. Hence, a two-level system cannot be made into a laser. Figure 2.2 shows absorption and emission of photons in a two level system.

\footnotetext{
${ }^{2}$ This is further simplification to the Bohr atomic model which was discussed earlier.

${ }^{3}$ Throughout this dissertation, ground level and ground state will be used interchangeably. Similarly, excited state and higher energy level will be used interchangeably.
} 


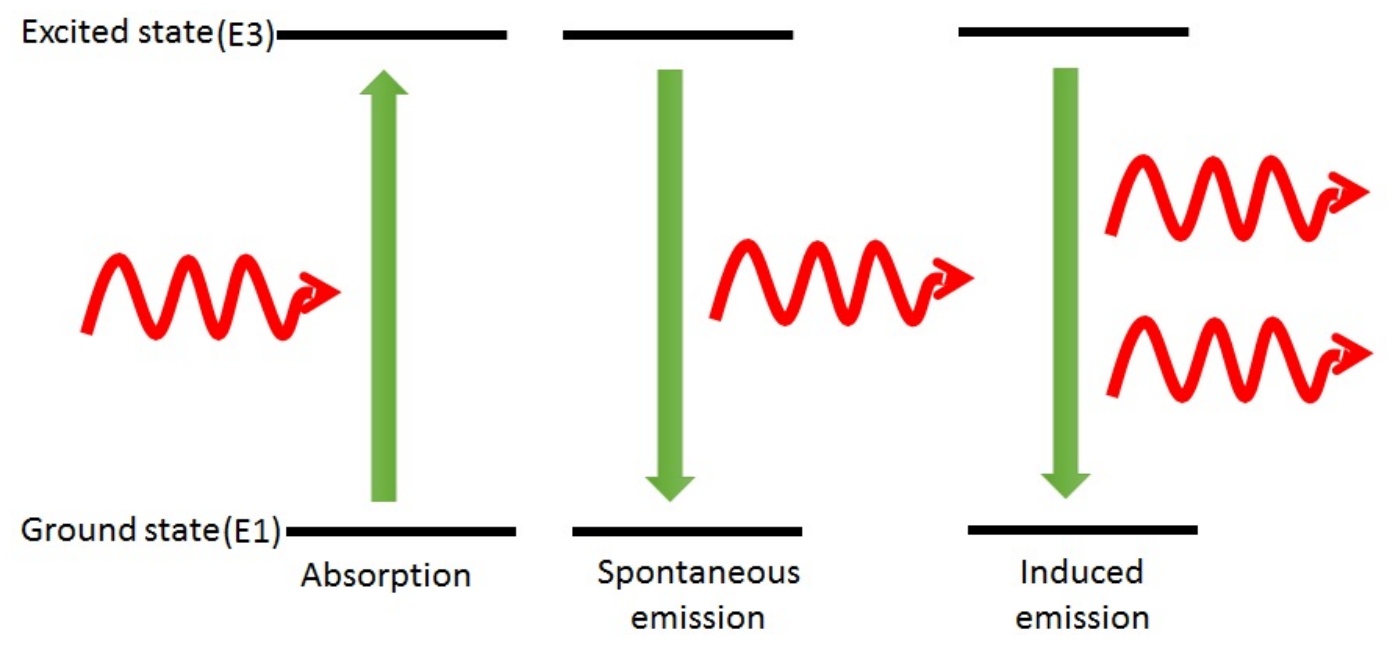

Figure 2.2: A two-level system

A three-level or a four-level system can be made into a laser by overcoming the limits to population inversion. In a three level system, as shown in figure 2.3 , the electrons in the gain medium are pumped into higher energy level (denoted by E3). These electrons can relax to the metastable state (denoted by E2) by generating phonons. Now, more and more electrons can be pumped into the excited states from the ground state. When the number of electrons in excited states exceeds the number of electrons in the ground state, it is called population inversion. The system becomes very unstable after the population inversion. In this situation, any addition of extra electrons to the excited state can stimulate the decay of all the excited electrons at once. These decaying electrons can emit photons with energy equivalent to the energy gap. This phenomenon is called stimulated emission. The amount of stimulated emission is larger than the amount of light absorbed. Hence, the light is considered to be amplified. When this optical amplifier is placed inside a resonant optical cavity as shown in figure 2.1, one obtains a laser oscillator also called a resonator. The lasing is possible only when the round trip gain of the active medium exceeds the round trip loss of the cavity. 


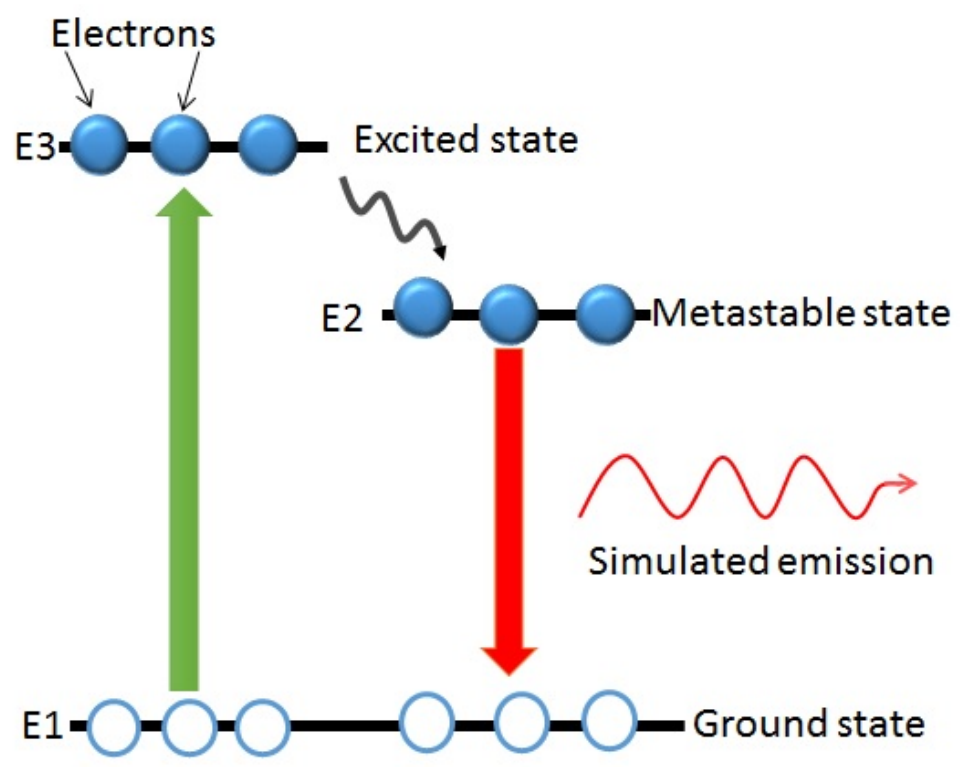

Figure 2.3: Energy diagram of a three-level laser system. Unfilled circle at the ground state corresponds to the vacancies after the removal of electrons.

The first working laser was demonstrated by Theodore Maiman in 1960, in which a high power flash lamp was shined on a ruby rod with silver-coated surfaces to initiate the lasing action [89]. Since then, there have been lots of advancements in the field of lasers. Lasers can be classified into two types: i) continuous wave (CW) laser and ii) pulsed laser. CW laser produces output that does not change as a function of time (see figure 2.4(a)). The output of a pulsed laser, also called modelocked laser, produces a periodic train of ultrashort pulses (see figure 2.4(b)). Active or passive mode locking technique can be applied to generate these laser pulses. A passively modelocked Ti:Sapphire laser for pump-probe spectroscopy was built. Hence, the dissertation will only discuss the passive modelocking in a Ti:Sapphire laser. 
(a)

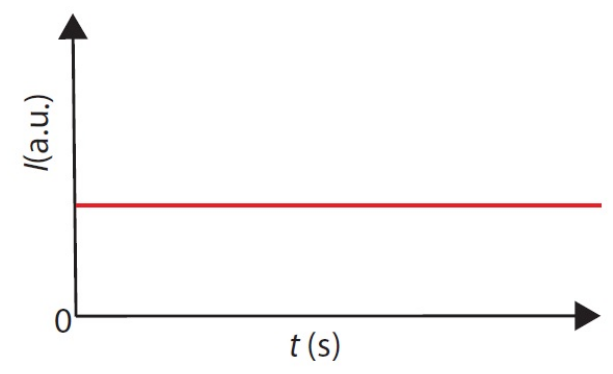

(b)

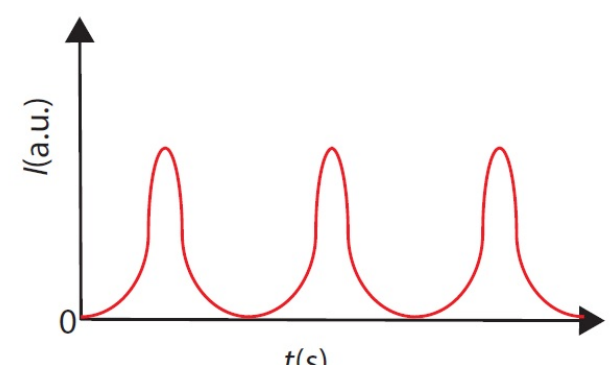

$t(\mathrm{~s})$

Figure 2.4: (a) CW mode and (b) mode locked (pulsed) output of a laser

\subsection{Ti:Sapphire Laser}

Ti:Sapphire laser is based in Titanium doped $\mathrm{Al}_{2} \mathrm{O}_{3}$ crystal. In order to produce a pulsed light, an active medium with large gain bandwidth is required. Since Ti:Sapphire has a huge gain bandwidth (660-986 nm), it is widely used as a gain

medium for generating ultrafast pulses of light with tunable wavelength [90] since adding of multiple modes allows shorter and intense pulse (more discussions is found in 2.2.1). Because of this versatility, the Ti:Sapphire laser has replaced most other kinds of laser (such as dye laser) since its introduction in 1986 [90]. A cartoon showing the energy level diagram of a Ti:Sapphire is depicted in figure 2.5. It is a four-level system. The lasing action occurs between the ${ }^{2} \mathrm{~T}_{2}$ triply degenerate ground state and the ${ }^{2} \mathrm{E}$ doubly degenerate excited state. The ground state is split due spinorbit interaction which results in a wide range of energies that can be obtained by decaying electrons. In figure 2.5, electron are optically pumped from A (ground) to $\mathrm{B}$ (excited). These electron relaxes to $\mathrm{C}$ by emitting phonons. At $\mathrm{C}$, the electrons do radiative decay to $\mathrm{D}$. The transition $\mathrm{C}$ to $\mathrm{D}$ is where the lasing action happens. Finally, the electrons relax back to A. 


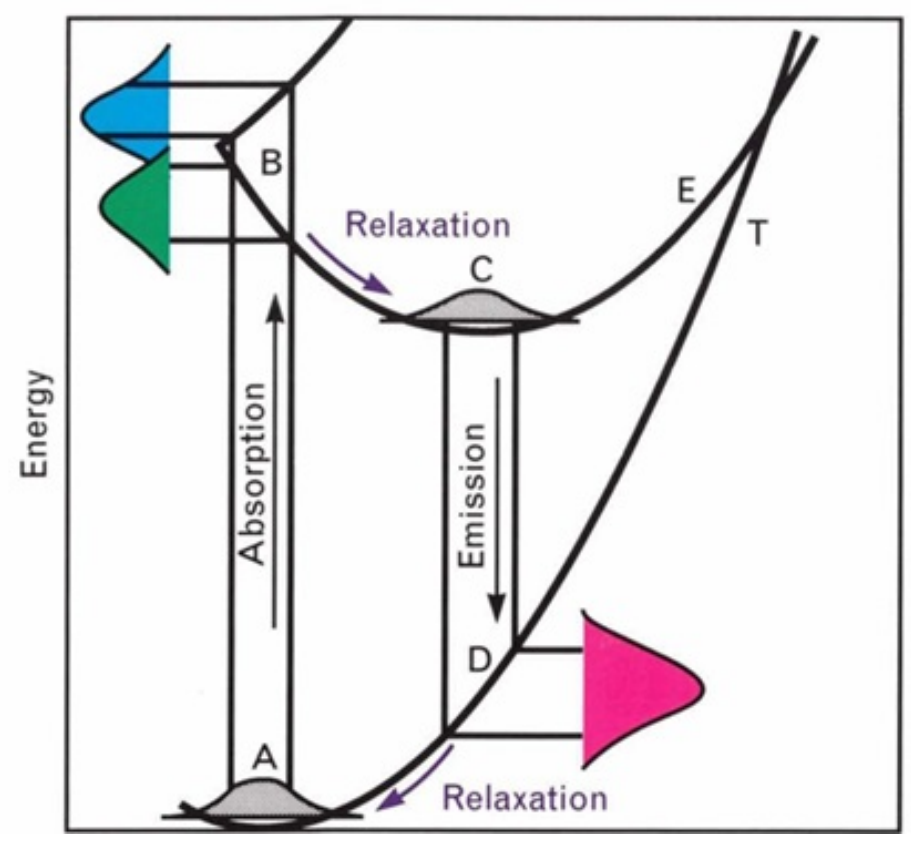

Figure 2.5: Energy level diagram of a Ti:Sapphire crystal showing lasing action [91].

Because of this high gain bandwith (emission bandwith), Ti:Sapphire laser can generate ultrashort pulses upto few femtoseconds [92]. The absorption and emission spectrum of a Ti:Sapphire crystal is given in figure 2.6. From the absorption spectra, it is clear that the laser can be pumped by a visible light source and the maximum of the laser output will be centered around $800 \mathrm{~nm}$. 


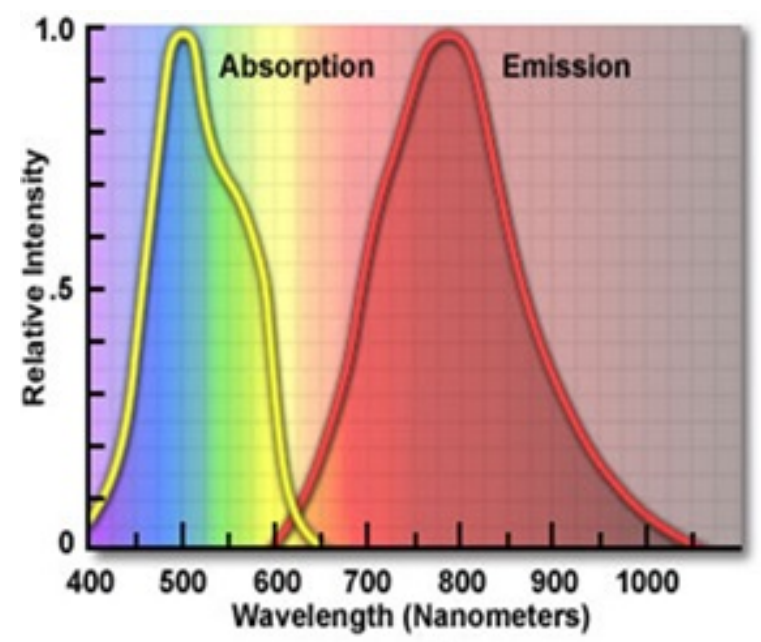

Figure 2.6: The absorption and emission spectrum of Ti:Sapphire crystal [93].

\subsubsection{Mode Locking}

Modelocking involves the "locking" together of the phases of many longitudinal modes in a resonator cavity [94]. Inside a laser cavity, there can be lots of longitudinal modes at one time, as long as they satisfy the boundary condition that is all the modes must vanish at the surface of the end mirrors. The phase locking of multiple longitudinal modes resulting in pulsed light can be modeled by a simple analog model resonator with multiple longitudinal modes. To simplify the math, the frequencies of the modes can be made to be equally spaced (given by $w_{n}=w_{0}+n \Delta$ ) and same amplitudes. $w_{0}$ is the central frequency. Where

$$
n=-\frac{N-1}{2},-\frac{N-1}{2}+1,,-\frac{N-1}{2}+2, \ldots ., \frac{N-1}{2}
$$

The output of this simple laser can be mathematically represented by

$$
E_{n}(t)=E_{0} \sin \left(w_{n} t+\phi_{0}\right)
$$


In above equation, $\phi_{0}$ is the phase. To satisfy the modelocking condition, all the frequency components must oscillate in phase. Now, the sum of all longitudinal modes can be given by,

$$
E(t)=\sum_{n} E_{n} t=-\sum_{(-N-1) / 2}^{(N-1) / 2} E_{0} \sin \left(w_{0} t+\phi_{0}\right)
$$

Using Euler's relation, one can rewrite

$$
E(t)=E_{0} \operatorname{Im}\left(\sum_{n} e^{i w_{0} t+\phi_{0}+n \Delta t}\right)=E_{0} \operatorname{Im}\left(e^{i\left(w_{0} t+\phi_{0}\right)} \sum_{n} e^{i n \Delta t}\right)
$$

where Im stands for imaginary part. Using the identity

$$
\begin{gathered}
\sum_{-(N-1) / 2}^{(N-1) / 2} e^{i n y}=\frac{\sin (N y / 2)}{\sin (y / 2)} \\
E(t)=E_{0} \operatorname{Im}\left[e^{w_{0} t+\phi_{0}} \frac{\sin (N \Delta t / 2)}{\sin (\Delta t / 2)}\right]=E_{0} \sin \left(w_{0} t+\phi_{0}\right)\left[\frac{\sin (N \Delta t / 2)}{\sin (\Delta t / 2)}\right] \\
E(t)=A_{N}(t) \sin \left(w_{0} t=\phi_{0}\right)
\end{gathered}
$$

The function $A_{N}$ can be plotted for $N=10, N=30$, and $N=50$ as shown in figure 2.7. As $N$ (the number of modes) increases, the amplitude also increases. 


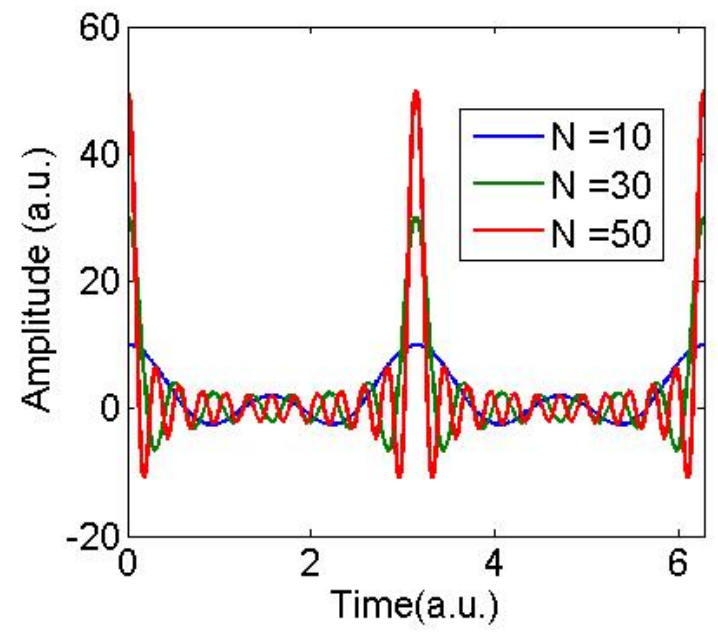

Figure 2.7: Electric field of a mode-locked laser pulse. Simulation is done using Matlab for number of modes $\mathrm{N}=10,30$, and 50 .

According to this model, the larger the number of modes, the narrower the pulse width, as shown in figure 2.7. The output light consists of pulse train separated by time $\left(T=\frac{2 L}{c}\right)$, where $L$ is the length of the cavity. The temporal duration of the pulse is given by $\tau_{p}=\frac{2 L}{c N}$ as shown in figure 2.8 .

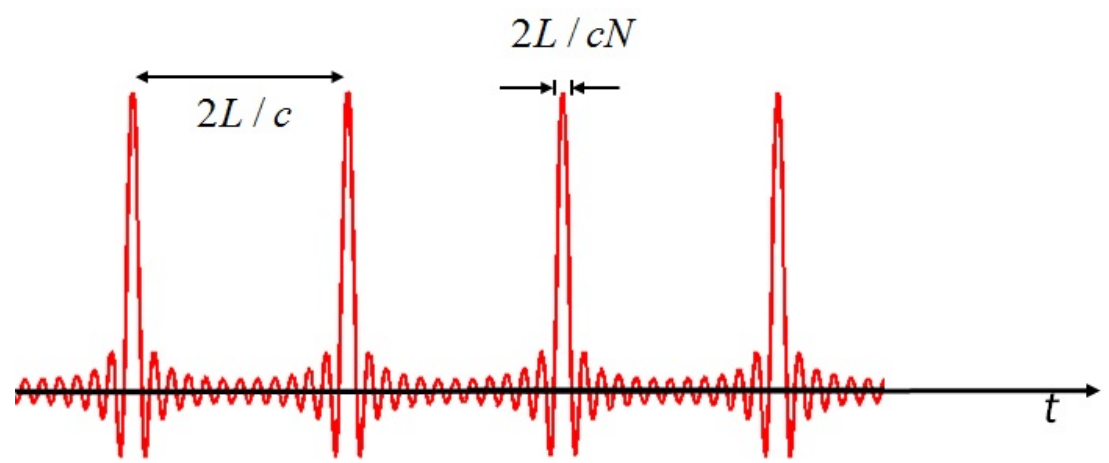

Figure 2.8: A modelocked pulsetrain as a function of time, observed at a fixed position. 


\section{Kerr-Lens Effect}

In order to modelock the laser for pulsed operation, Kerr lensing effect of the Ti:Sapphire crystal is exploited. Kerr-lens modelocking is a passive modelocking technique. In this technique, the gain material (Ti:Sapphire crystal for the current laser setup) acts as a lens which focuses the beam even further at higher laser intensities. The Kerr lens effect is a nonlinear optical effect that creates an intensitydependent refractive index in a material. Mathematically [95],

$$
n=n_{0}+n_{2} I(r, t)
$$

where, $n_{0}$ is the linear refractive index, $n_{2}$ is the non-linear refractive index, and $I(r, t)$ the radial time dependent intensity of the laser pulse. The refractive index will increase with increase in pump intensity. Because the pump intensity is not spatially uniform, the Kerr effect will be more significant at the center of the medium where the beam is more intense. This nonuniform refractive index forms a gradient index lens that self-focusses the more intense portion of the beam [95] (see figure 2.9). Hence, using this effect, generation of pulse with very short time duration is possible.

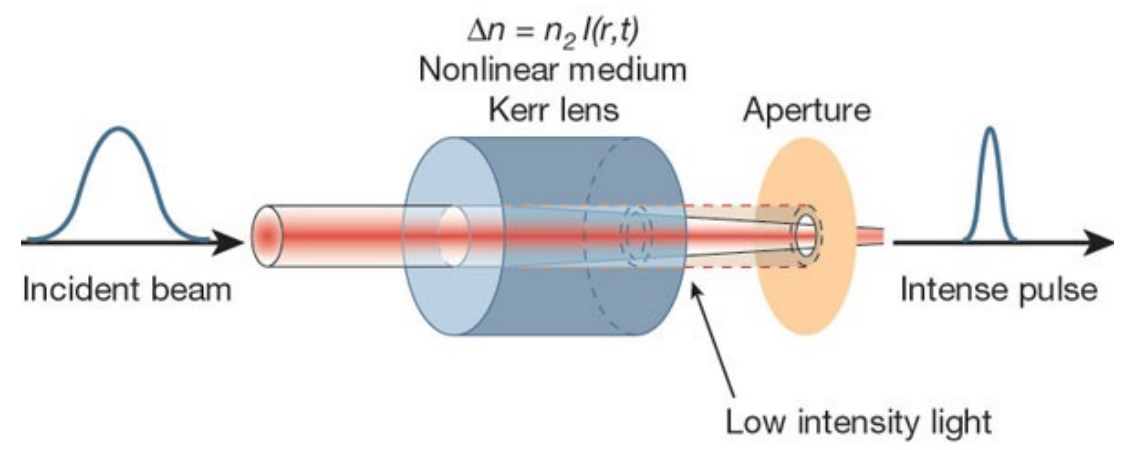

Figure 2.9: Kerr lens modelocking mechanism in Ti:Sapphire laser [95]. 


\subsubsection{Cavity Design}

As mentioned earlier (see figure 2.6) the peak absorption of the Ti:Sapphire crystal is around 500nm. Hence, the home built Ti:Sapphire laser is pumped by a commercial CW laser from Lighthouse Pohotonics (Sprout G) which has maximum output power of $10 \mathrm{~W}$ with $533 \mathrm{~nm}$ wavelength. The Ti:Sapphire oscillator cavity consists of $2.5 \mathrm{~mm}$ thick Ti:Sapphire crystal, $150 \mathrm{~mm}$ plano-convex lens (L), a pair of focusing mirrors (F1 and F2) with radius of $75 \mathrm{~mm}, 2$ pair of cavity mirrors (M1, M2, M3 , and M4), a 99\% reflection high reflector (HR,) and a $10 \%$ transmission output coupler (OC) (see figure 2.10). Table 2.1 shows all the cavity parameters and their values. The cavity has a total length of $203.33 \mathrm{~cm}$ and is asymmetric, with $130.43 \mathrm{~cm}$ in one arm of the laser and $72.9 \mathrm{~cm}$ on the other arm. The asymmetric cavity arrangement is very important for production of a high-power $T E M_{00}$ modelocked spatial mode [96]. More information on the initial cavity alignment and the troubleshooting of the cavity is included in appendix A and B.

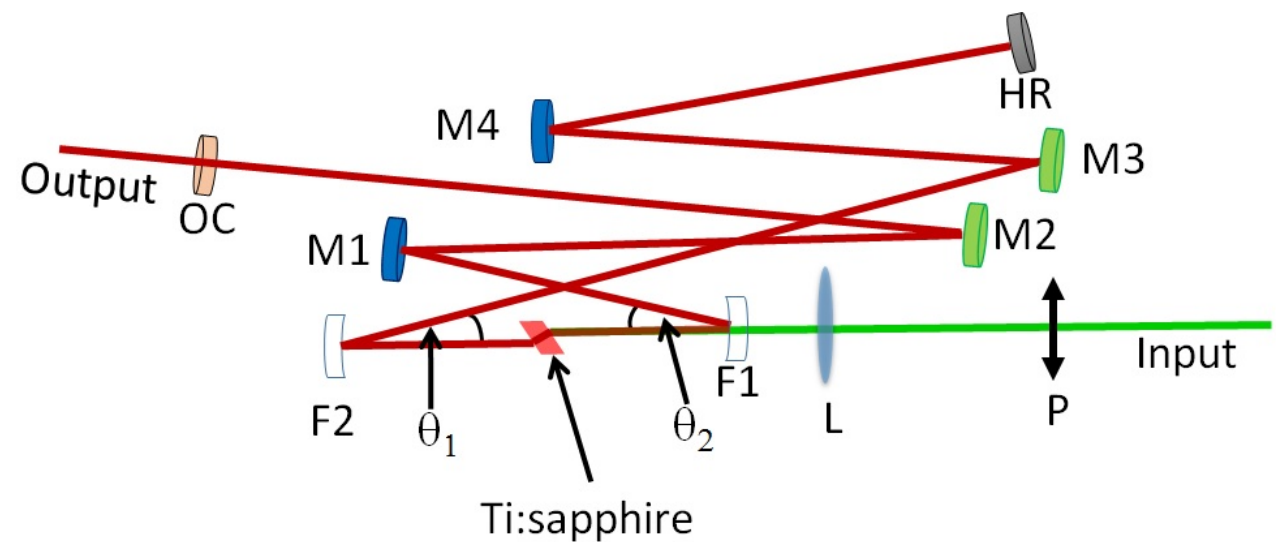

Figure 2.10: A Ti:Sapphire laser cavity 


\begin{tabular}{|c|c|}
\hline Parameters & Values \\
\hline Focal length of the lens (L) & $150 \mathrm{~mm}$ \\
\hline Focusing mirror (F1 and F2) & $75 \mathrm{~mm}$ \\
\hline Crystal thickness & $2.5 \mathrm{~mm}$ \\
\hline Refractive index of Ti:Sapphire & $1.76[97]$ \\
\hline Arm 1 length (Crystal-F2-M3-M4-HR) & $130.43 \mathrm{~cm}$ \\
\hline Arm 2 length (Crystal-F1-M1-M2-OC) & $72.9 \mathrm{~cm}$ \\
\hline$\theta_{1}$ & $11.4^{\circ}$ \\
\hline$\theta_{1}$ & $9.5^{\circ}$ \\
\hline
\end{tabular}

Table 2.1: Laser cavity parameters

In order to generate stable ultrashort pulses, the positive group velocity dispersion (GVD) introduced by the optics in the cavity should be compensated by other optics with negative GVD [98]. GVD is a phenomenon that causes a short light pulse to spread in time because different frequency components of a light pulse travels at different velocities. Mathematically, it is given by:

$$
G V D=\frac{\partial}{\partial \omega} \frac{1}{v_{g}}=\frac{\partial}{\partial \omega}\left[\frac{\partial k}{\partial \omega}\right]=\frac{\partial^{2} k}{\partial \omega^{2}}
$$

where, $v_{g}$ is the group velocity, $\omega$ is the frequency, and $k$ is the wavenumber. In the past, prism pairs were used for the GVD compensation [99]. Now, high damagethreshold dispersion compensating mirrors (DCM), with high reflectivity and a nearly constant negative GVD over frequency ranges that has small footprint, are commercially available ${ }^{4}$. These mirrors are made with multilayer dielectric coatings so that

\footnotetext{
${ }^{4}$ Some of the companies selling these DCM mirrors are Newport, Venteon, Thorlabs, etc
} 
different wavelengths can have different penetration lengths, hence the different group delays. The coating layers are tailored to achieve a net negative GVD [100]. In this home built Ti:Sapphire laser, DCM pairs are used in the cavity which provides overall negative GVD. Furthermore, the focusing mirrors also have dielectric coatings to provide negative GVD. Ti:Sapphire crystal and the OC provides positive GVD which is compensated by the negative GVD. Given below (Table: 2.2) is a table with Group Delay Dispersion (GDD) from each optics in the cavity ${ }^{5}$. GVD is GDD per unit length of the material. In our laser cavity the total GDD is slightly negative.

\begin{tabular}{|c|c|c|c|}
\hline Cavity Optics & GDD $\left(f s^{2}\right)$ & company & part \# \\
\hline F1 and F2 & -50 & Layertec & 106234 \\
\hline Ti:Sapphire crystal & $170[101]$ & Crystal System & N/A \\
\hline Cavity Mirrors M1 and M2 & -100 & Venteon & DCM7 \\
\hline Cavity Mirrors M3 and M4 & -100 & Venteon & DCM7 \\
\hline HR & $\sim 0$ & Femtolaser & OA121 \\
\hline OC & 30 & Layertec & 101905 \\
\hline
\end{tabular}

Table 2.2: Estimation of GDD by the optics inside of the laser cavity.

The laser outputs pulses with about $35 \mathrm{~nm}$ (FWHM) bandwidth (Figure 2.11). The central wavelength is around $840 \mathrm{~nm}$. The central wavelength can be tuned from $800 \mathrm{~nm}$ to $850 \mathrm{~nm}$ by adjusting the cavity alignment.

\footnotetext{
${ }^{5}$ Except for the Ti:Sapphire crystal, the GDD values are acquired from the parts suppliers. GDD reported are in the Ti:Sapphire wavelength range. The GDD approximation for all the optics is done for one pass.
} 


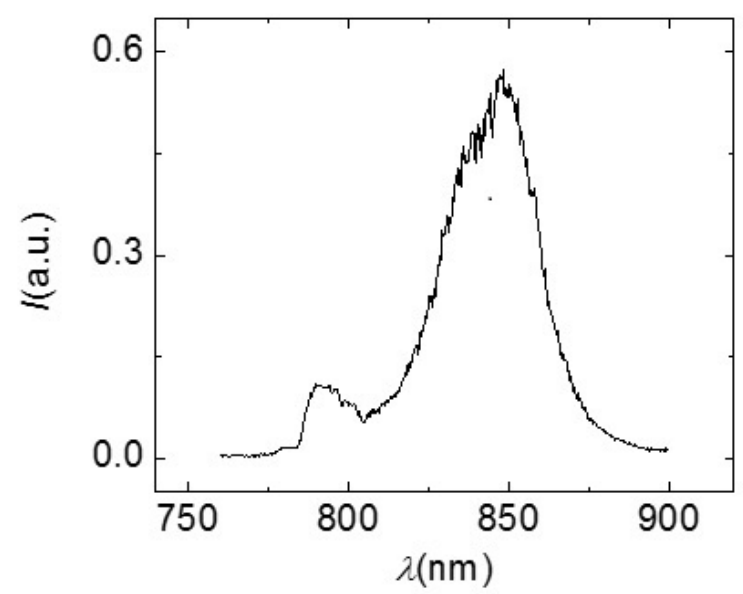

Figure 2.11: Spectrum of the laser output as measured by a spectrometer. The laser is capable of lasing from $800 \mathrm{~nm}$ to $850 \mathrm{~nm}$. The peak around 800 corresponds to another lasing position.

By measuring the pulse duration with a Frequency-resolved Optical Gating (FROG), the pulsewidth was estimated to be around 30 fs (Figure 2.12). The concept of FROG is explained in detail in appendix $\mathrm{C}$.

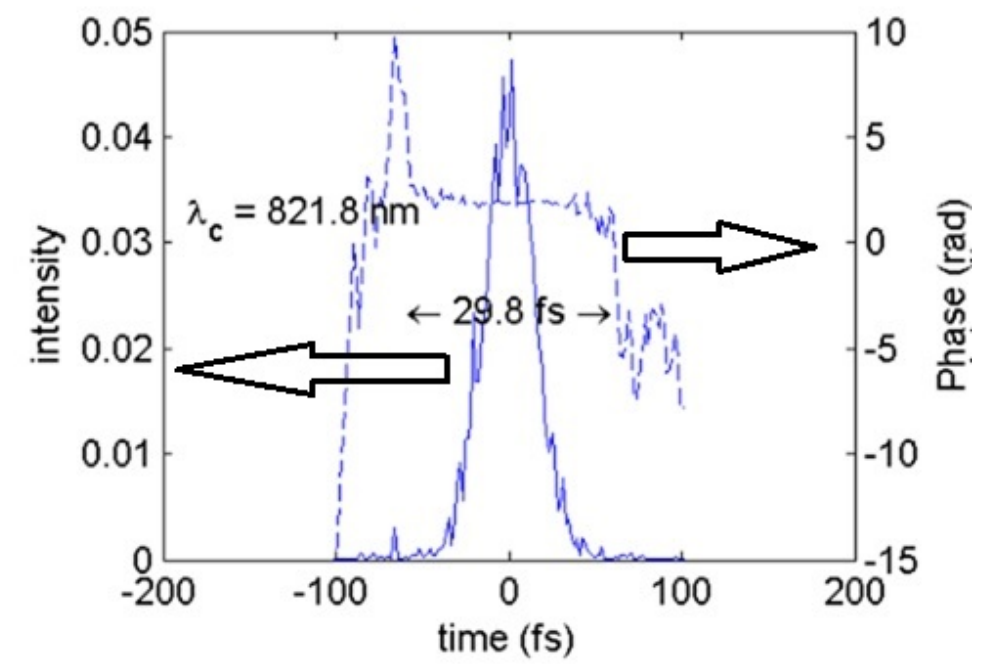

Figure 2.12: Pulsewidth of the laser outup as measured by FROG. 
The repetition rate of the laser when measured with a fast photodetector is 74 MHz (Figure 2.13). The total length of the cavity $L$ is $203.33 \mathrm{~cm}$. Theoritically, this should correspond to repetition rate of:

$$
\nu=\frac{c}{2 L}=\frac{3 \times 10^{8} \mathrm{~m} / \mathrm{s}}{2 \times 2.0333 \mathrm{~m}}=74 \mathrm{MHz}
$$

Experimentally measured repetition rate exactly matches with the repetition calculated from the cavity length.

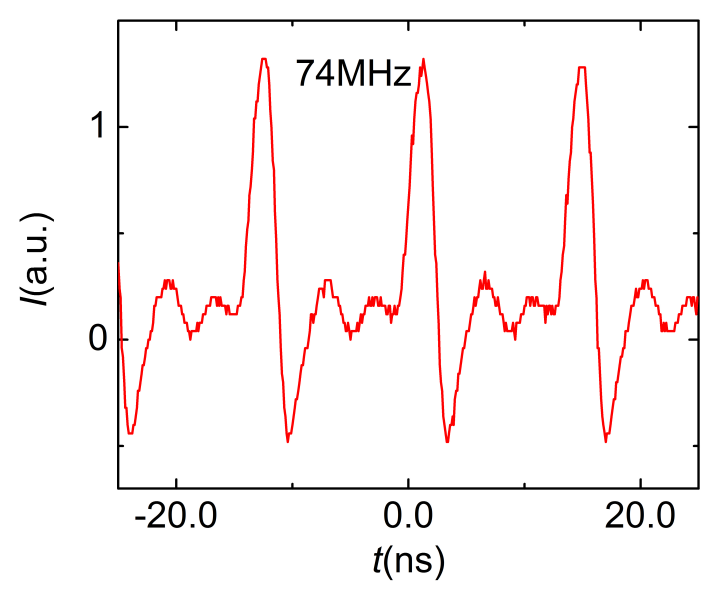

Figure 2.13: Pulsetrain of the oscillator measured using a fast detector connected to a high bandwidth oscilloscope.

\subsubsection{Applications}

A modelocked laser has lots of applications in nonlinear optics, such as secondharmonic generation [102], parametric down-conversion [103], optical parametric oscillators [104], and generation of Terahertz radiation [105], which can be used in many different applications. Also, a modelocked laser is required in ultrafast spectroscopy such as, pump probe spectroscopy [106], time resolved x-ray photoemission spectroscopy [107], time-domain terahertz spectroscopy [108], ultrafast electron diffrac- 
tion [109], etc. A pump-probe spectroscopy system was built and used to study one of the material studied for this dissertation. Hence, pump-probe spectroscopy will be explained in detail, in the next chapter. 


\section{Chapter 3}

\section{Pump-probe Spectroscopy}

Pump-probe spectroscopy is an experimental technique of probing a non-equilibrium system, using an optical pulse, to understand the electronic relaxation dynamics and processes after optical excitation by a pump pulse. Thus, a pump pulse excites or modifies the system and a probe pulse measures the excitation as a function of time delay between pump and probe pulses. A pulsed light source is a must for this kind of spectroscopy. Since the pulsewidth of the pump and probe are in femtosecond ${ }^{1}$ timescale, one can observe the temporal evolution of a pumped system with femtosecond resolution.

\subsection{Time Resolution}

Mathematically, the temporal resolution of a pump-probe system can be calculated from the width of the convolution integral of pump and probe pulse which can

\footnotetext{
${ }^{1} 1$ femtosecond is $10^{-15}$ second.
} 
be approximated by two Gaussian signal [110]:

$$
f_{c}(t)=\int_{-\infty}^{+\infty} f_{\text {pump }}(\tau) f_{\text {probe }}(\tau+t) d \tau
$$

where $f_{\text {pump }}(t)$ is the Gaussian pump pulse and $f_{\text {probe }}(t)$ is the probe pulse. Gaussian

function is given by $f(t)=\exp \left[-\frac{t^{2}}{\Delta t^{2}}\right]$, where $\Delta t$ is the pulse with. Then the convolution integral can be given by

$$
\begin{gathered}
f_{c}(t)=\int_{-\infty}^{+\infty} \exp \left[-\frac{\tau^{2}}{\Delta t_{\text {pump }}^{2}}\right] \exp \left[-\frac{(\tau+t)^{2}}{\Delta t_{\text {probe }}^{2}}\right] \\
f_{c}(t)=\frac{\sqrt{\pi} \Delta t_{\text {pump }} \Delta t_{\text {probe }}}{\sqrt{\Delta t_{\text {pump }}^{2}+\Delta t_{\text {probe }}^{2}}} \exp \left[-\frac{t^{2}}{\Delta t_{\text {pump }}^{2}+\Delta t_{\text {probe }}^{2}}\right]
\end{gathered}
$$

Thus, the pulsewidth of the convoluted function can be given by:

$$
\Delta T=\sqrt{\left(\Delta T_{\text {pump }}\right)^{2}+\left(\Delta T_{\text {probe }}\right)^{2}}
$$

where, $\Delta T_{\text {pump }}$ and $\Delta T_{\text {probe }}$ are the pulsewidth of the pump and probe pulse. $\Delta T$ can also be called the temporal resolution of the pump-probe spectroscopy system which depends on the pulsewidth of the pump and probe. Hence, the temporal resolution of a pump-probe system depends on the pulsewidth of the pump and probe pulse.

\subsection{Ultrafast Processes}

In a material at equilibrium, energy is exchanged through carrier-carrier and carrier-phonon interactions, keeping the carriers and phonons at a common temperature bath. However, the absorption of pump by electrons disrupts this equilibrium 
state and creates "hot" carriers. Holes are created in the position where electron are excited. These electrons and holes would like to recombine eventually. Many dynamical process can happen in-between the initial excitation and the eventual recombination. They are: 1) electron-electron scattering, 2) electron-phonon scattering, 3) radiative recombination, 4) non-radiative recombination, and so on. Figure 3.1 shows the electron-electron scattering during the relaxation.

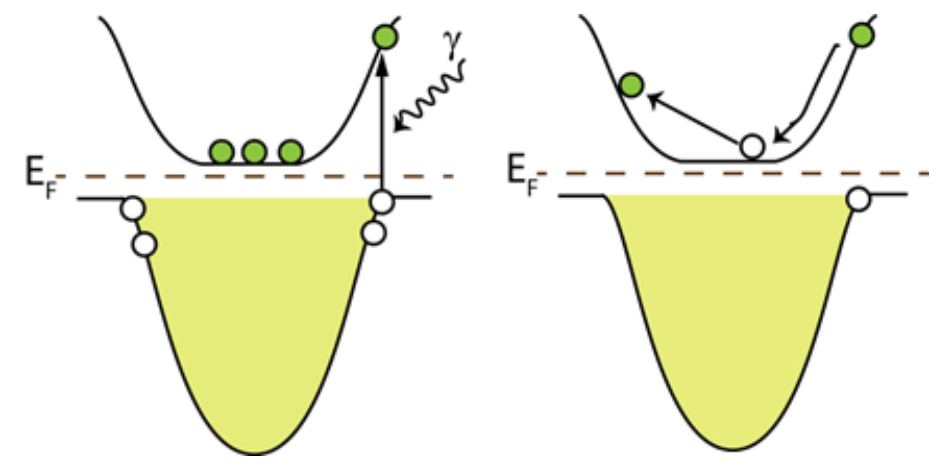

Figure 3.1: Cartoons showing light matter interactions in a material with small gap. Figure on the left shows creation of "hot" carriers due to pump and figure on the right shows thermalization of the hot carriers with electron-electron scattering.

All these dynamical processes are in ultrafast time scale ${ }^{2}$. In order to resolve these processes, ultrafast probing is required because even a fast detector does not have enough resolution. Relaxation through phonons involves various scattering processes. In this process the average carrier temperature relaxes to the lattice temperature [106]. Some of the other ultrafast process that require high resolution probing system to study them are given below (see figure 3.2).

\footnotetext{
${ }^{2}$ Ultrafast corresponds to time shorter than picosecond $\left(10^{-12}\right.$ second).
} 


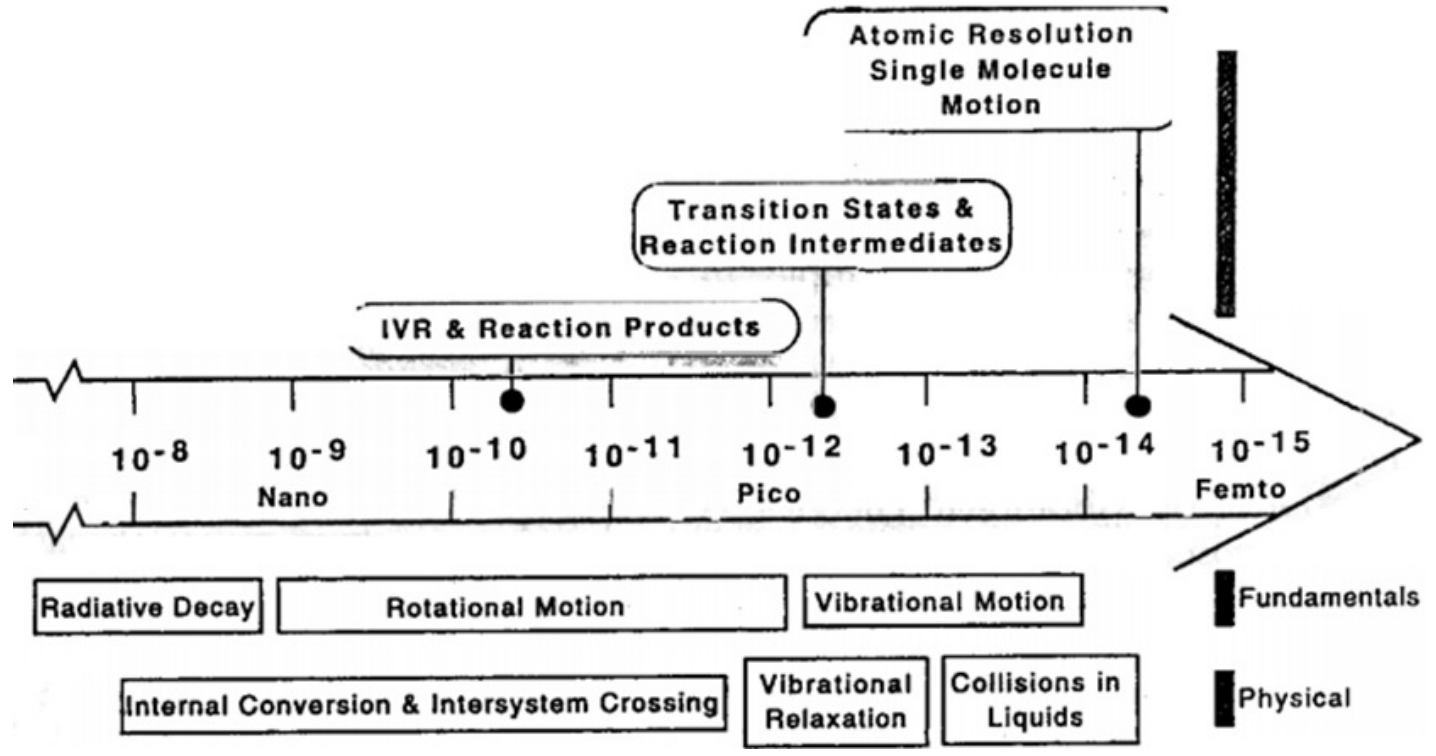

Figure 3.2: Time scales of some of the ultrafast phenomenons [111] that requires ultrafast time resolution to resolve.

\subsection{Photoinduced Reflectivity Change}

Reflectivity change in a material can be influenced by: 1) change in carrier density, 2) renormalization of the band gap, and 3) lattice heating (lattice temperature change) [112]. A pump light can influence all these properties of a material. So, a probe can measure the dynamical change in the reflectivity of the material due to the pump by probing the material with a probe pulse at different time delay. Figure 3.3 shows an example of change in reflectivity in ultrafast time domain. 


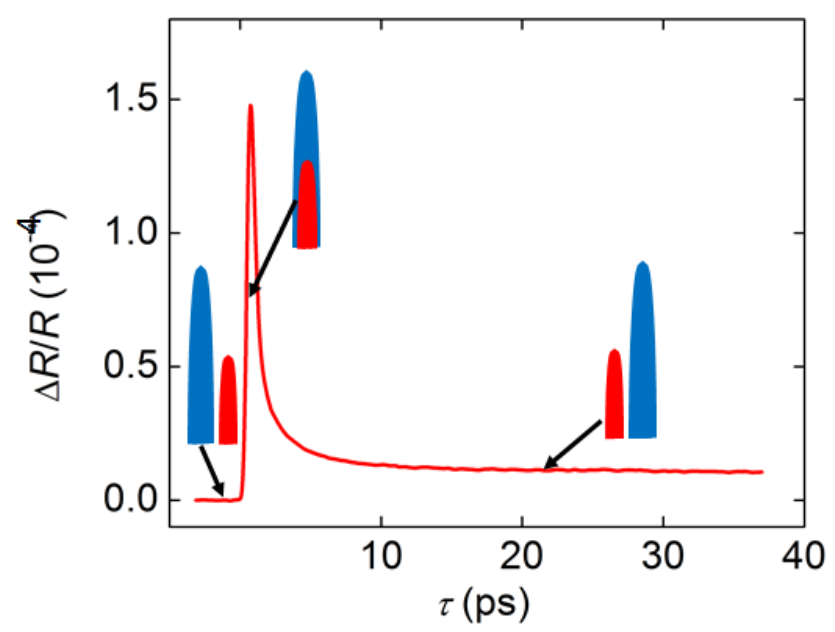

Figure 3.3: Example of an ultrafast photoinduced reflectivity measurement at different delay. Before zero delay, the probe pulse ( in red with smaller amplitude) arrives at the sample before the pump pulse ( in blue with higher amplitude). At zero delay the pump and probe arrive at the exact time. After the zero delay, the probe arrive after the pump and measures the carrier relaxation dynamics.

\subsubsection{Optical Reflectance}

For light incident at normal, reflectivity coefficient $r(\omega)$ can be given by the ratio of the reflected electric field to the incident electric field.

$$
r(\omega)=\frac{E(\text { ref })}{E(\text { inc })}=\frac{\tilde{n}+i \tilde{\kappa}-1}{\tilde{n}+i \tilde{\kappa}+1}
$$

where, $\tilde{n}$ is the referactive index and $\tilde{\kappa}$ is the extinction coefficient. By definition:

$$
\sqrt{\epsilon(\omega)} \equiv \tilde{n}+i \tilde{\kappa}
$$


where, $\omega$ is the frequency of the light.

$$
r(\omega)=\frac{\sqrt{\epsilon(\omega)}-1}{\sqrt{\epsilon(\omega)}+1}
$$

According to Drude theory, free carrier play very important role in the conductivity of metals and semiconductors. In this model, the total complex dielectric function can be given by:

$$
\epsilon(\omega)=\epsilon_{\text {core }}(\omega)+\frac{4 \pi i \sigma}{\omega}
$$

where, $\epsilon_{\text {core }}$ is the contribution to the permittivity due to the core electron.

$$
\sigma=\left[\frac{n e^{2} \tau}{m^{*}}\right] \frac{1}{1-i \omega \tau}
$$

In above equation, $\tau$ is the relaxation time, $m^{*}$ is the effective mass, $e$ is the electron charge, and $n$ is the carrier density. For material with gap the contribution of holes to the conduction is same as the electron. Now, the complex dielectric constant can be generalized to:

$$
\epsilon=\epsilon_{\text {core }}+\frac{4 \pi i}{\omega}\left[\frac{n_{e} e^{2} \tau_{e}}{m_{e}\left(1-i \omega \tau_{e}\right)}+\frac{n_{h} e^{2} \tau_{h}}{m_{h}\left(1-i \omega \tau_{h}\right)}\right]
$$

From above derivation, it can be concluded that optical reflectivity measurement can give information about the carrier. Hence, change in reflectivity of the probe can measures the dynamical behavior of the carriers due to the pump at different times. 


\subsection{Experimental Setup}

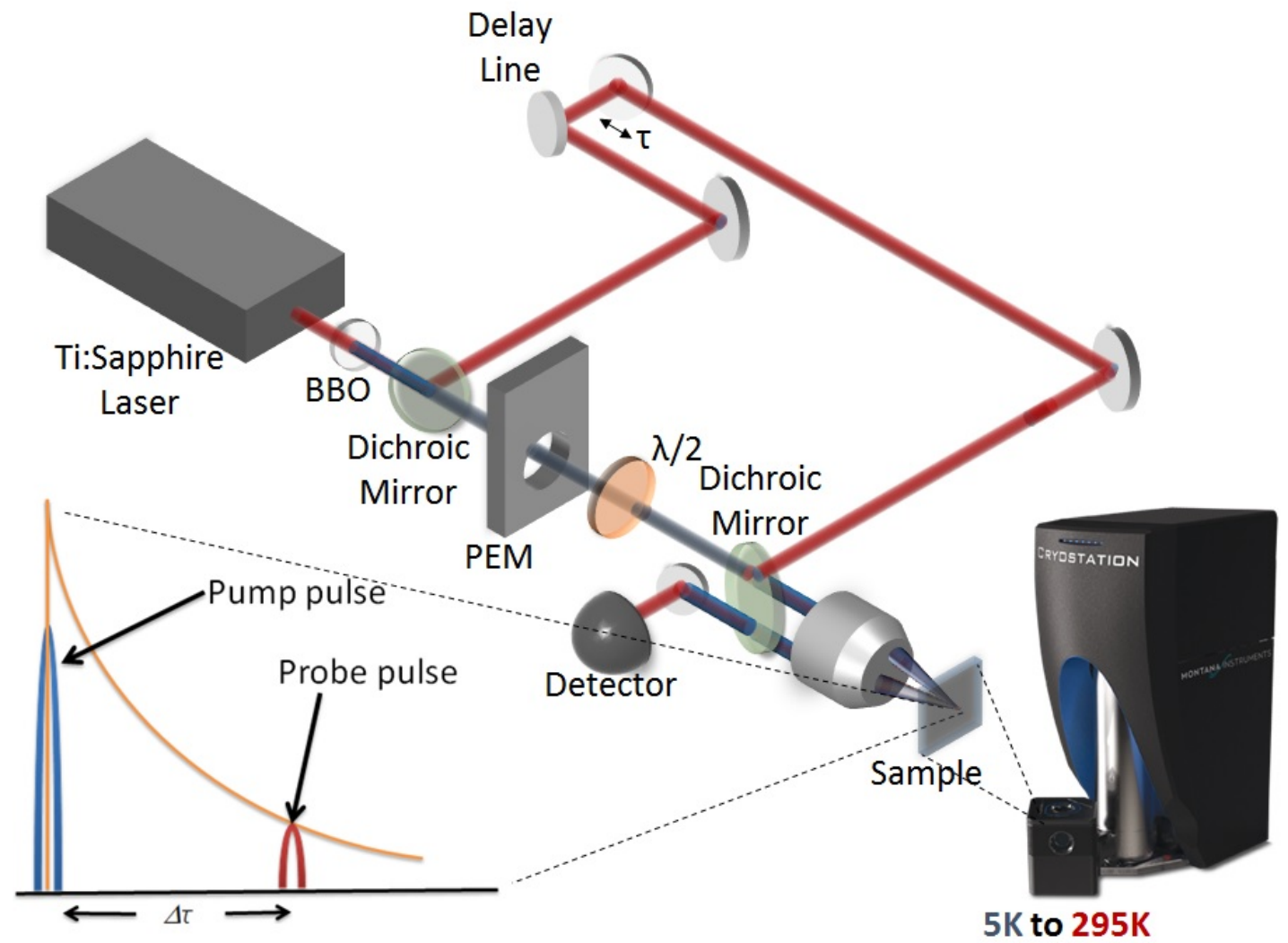

Figure 3.4: Pump-probe spectroscopy setup

The pump-probe spectroscopy setup consists of a homemade Ti:Sapphire oscillator producing $30 \mathrm{fs}$ pulses centered at $840 \mathrm{~nm}(1.48 \mathrm{eV})$ with $74 \mathrm{MHz}$ repetition rate (see chapter 2 for more details). The output of this oscillator pumps a Barium Beta Borate (BBO) crystal to generate a second harmonic pulse centered at $420 \mathrm{~nm}$ $(2.96 \mathrm{eV})$. Both pulses, the $420 \mathrm{~nm}$ pump and the $840 \mathrm{~nm}$ probe are focused onto the sample surface with an aspheric lens or objective. The pump and probe pulses are delayed with respect to one another using a translation stage which is controlled by LabView (from a computer). Pump intensity is modulated at $84 \mathrm{kHz}$ by a photoelastic modulator (PEM). The sample-reflected probe intensity $(\mathrm{R})$ is acquired using 
a detector. A lock-in amplifier is used to filter out frequencies other than the pump induced (PI) reflectivity change $(\Delta R / R)$ by locking into the PEM frequency. The detector used is a balanced detector which further suppress the background noise. Sample temperature is varied between $5 \mathrm{~K}$ and $294 \mathrm{~K}$ in a close cycle cryostation. Each component of the experimental setup are explained in appendix D. 


\section{Chapter 4}

\section{Ultrafast observation of electron}

\section{hybridization and in-gap states \\ formation in Kondo insulator}

\section{$\mathrm{SmB}_{6}$}

\subsection{Abstract}

$\mathrm{SmB}_{6}$ is a promising candidate for topological Kondo insulator. Here, we report ultrafast carrier dynamics of $\mathrm{SmB}_{6}$. Two characteristic temperatures: $T_{1}=100 \mathrm{~K}$ and $T_{2}=20 \mathrm{~K}$ are observed. $T_{1}$ corresponds to the opening of the $f-d$ hybridization gap revealed by an abrupt disappearance of terahertz $f$-band plasmon oscillations. Between $T_{1}$ and $T_{2}$, a "phonon bottleneck" effect dominates the photocarrier relaxation processes. Below $T_{2}$, we observe the formation of in-gap states formation and faster relaxation of the carriers. 


\subsection{Introduction}

Kondo insulators (KI), in particular $\mathrm{SmB}_{6}$, are compounds in which strong correlation effects give rise to an insulating ground state at low temperatures. In general, the strong interaction within the localized periodic dense array of $f$-magneticmoments (so-called Kondo lattice) leads to reconstructions of the electronic structure and opens up [113] an energy gap at low temperatures due to the hybridization between conduction electrons and the highly renormalized $f$-electrons. When the Fermi level lies within the gap, a KI is formed. Recent theories of topological Kondo insulator (TKI) $[8,12-21]$ have predicted, in $\mathrm{SmB}_{6}$, the existence of a topological nontrivial surface state with odd number of Dirac surface bands. The hybridization and odd parity wavefunction lead to strong spin-orbit coupling and give rise to a topological surface state that dominates electron conduction at low temperatures. $\mathrm{SmB}_{6}$ is also predicted to be a strong topological insulator (TI) $[8,13]$. First principle calculations $[13-16,18-20]$ have proposed a surface state with three Dirac bands at $\Gamma$ and $\mathrm{X} / \mathrm{Y}$ points in the (100) surface.

In the past few years, tremendous experimental progress has been made regarding $\mathrm{SmB}_{6}$. Saturation of resistance at very low temperature from many different kinds of transport measurements has revealed the surface layer $[2,9,10,24,25]$. Point-contact

spectroscopy [26], scanning tunneling spectroscopy (STS) [27,28], angle-resolved photoemission spectroscopy (ARPES) [26, 29, 29-32] and magnetometry [33] provided further evidences of the surface state. Sensitivity to magnetic dopants, Dirac-like dispersion and spin helicity have been discovered $[2,26,29,33]$ in support of the TKI theory. In the meanwhile, controversies still exist. Non-topological surface states associated with the Boron dangling bond, common in hexaborides [34,35], bring additional complexities to the surface states interpretation. Evidences of surface Boron 
suboxide formation [36], Fermi level shift at polar and non-polar surfaces [37] and polarity driven surface metallicity [38] were found in $\mathrm{SmB}_{6}$. Metallic bulk states [39] and impurity states based carrier hopping mechanism due to valance-fluctuation between $\mathrm{Sm}^{2+}\left(4 f^{6}\right)$ and $\mathrm{Sm}^{3+}\left(4 f^{5} 5 d^{1}\right)$ configurations [40] were also suggested as alternative contributors to the low temperature resistance plateau. The entanglement of surface state topology with strongly correlated bulk states, as well as the complicated surface chemistry, makes $\mathrm{SmB}_{6}$ a highly attractive system for more in depth investigations.

Most of the measurements mentioned above address equilibrium states, where material properties are often described by effective thermodynamic parameters. On the contrary, pump-probe technique abruptly disturbs the material equilibrium by laser pulses and studies the following ultrafast re-equilibrium process as a function of time. By examining various characteristic dynamics in time domain, this technique can help distinguish many intrinsic degrees of freedom that are entangled at equilibrium. Examples include the study of electron-phonon interaction [41-44], order parameter fluctuation [45], pseudogap formation [46], and spin-orbit coupling [47] in many strongly correlated materials. In $\mathrm{SmB}_{6}$, besides the pioneering work by Demsar. et al. [42], recent works of time resolved Terahertz spectroscopy [48-50] and pump-probe photoemission spectroscopy [51] have also provided valuable insights into the low temperature band structure and the surface band bending. In this letter, photoexcited electron dynamics in $\mathrm{SmB}_{6}$ are investigated by optical pump-probe technique. Observations of the characteristic $f$-electrons plasmon help pin-point the hybridization gap opening temperature. Probing the photocarrier relaxation in time domain allows us to quantify the gap and sensitively detect the emergence of in-gap states at much lower temperature. To evaluate the robustness of the observed prop- 
erties, experiments are performed on two types of samples with distinct crystalline characters: single crystal and polycrystalline (as grown) thin film.

\subsection{Experimental Setup}

$\mathrm{SmB}_{6}$ single crystals were grown using the aluminum flux method similar to previous works $[2,24,25]$. Thin films of $\mathrm{SmB}_{6}$ are deposited on top of $\mathrm{MgO}$ substrates by pulsed laser deposition (PLD) (see section 4.4). Resistances in both single crystal and thin film samples saturate below $4 \mathrm{~K}$ (Figure 4.7), indicating an insulating bulk and the possible formation of surface states at low temperatures $[2,24]$. Our pumpprobe setup consists of $35 \mathrm{fs}$ probe pulses centered at $840 \mathrm{~nm}$ with a $74 \mathrm{MHz}$ repetition rate, and pump pulses from their second harmonic (420 nm). Both pump and probe are focused to the sample surface with a single aspheric lens. Sample temperature is varied between $5 \mathrm{~K}$ and $294 \mathrm{~K}$ in a close cycle cryostation with pressure maintained at $10^{-5}$ mbar level. All the data we present here are robust against full range thermal cycles.

\subsection{Sample Synthesis}

All the sample for the experiments performed in this chapter are acquired from collaborators. Single crystal of $\mathrm{SmB}_{6}$ were acquired from Zachery Fisk and Jing Xia (University of California, Irvine). $\mathrm{SmB}_{6}$ single crystals were grown using the aluminum flux method. These crystals are then inspected using X-ray analysis to make sure $\mathrm{SmB}_{6}$ is the only content. To further ensure that there is no remaining aluminum inside the crystal, we perform susceptibility measurements to check any sign of superconductivity of aluminum. The surfaces of these crystals were care- 
fully etched using hydrochloric acid (50 HCL +50 DI water) for 2 minutes and then cleaned using solvents to remove the possible oxide layer. Samples used in the experiments have naturally existing well defined (100) surfaces that are a few $\mathrm{mm}$ in size. We have carried out transport measurements of crystals grown from the same batch and make sure there is low temperature resistance saturation, a sign of surface-dominated conduction.

Thin film of $\mathrm{SmB}_{6}$ were acquired form Yanjun Ma and Chang-Beom Eom(University of Wisconsin, Madison). Thin film of $\mathrm{SmB}_{6}$ is synthesized by pulsed laser deposition (PLD). A KrF excimer laser outputs $248 \mathrm{~nm}$ laser pulses, which is focused on a $\mathrm{SmB}_{6}$ ceramic target with the spot size of $2.8 \mathrm{~cm}^{2}$ in the deposition chamber. The laser repetition rate is $10 \mathrm{~Hz}$, and the energy density is $3.6 \mathrm{~J} / \mathrm{cm}^{2}$. The ablated $\mathrm{SmB}_{6}$ materials deposit on a $\mathrm{MgO}$ (100) single crystal substrate, of which the surface is polished and the temperature is kept at $850{ }^{\circ} \mathrm{C}$. The working distance is $6 \mathrm{~cm}$. The growth pressure is maintained at $1 \times 10^{-7}$ Torr during the deposition. Right after growth, X-ray diffraction on the film reveals its polycrystalline structure; however, it is also noticed that the film changes to amorphous phase over time even when it's stored in a desiccator inside which the temperature is set to be about $20{ }^{0} \mathrm{C}$ and the humidity is controlled around $10 \%$. 


\subsection{Data and Discussion}

(a)

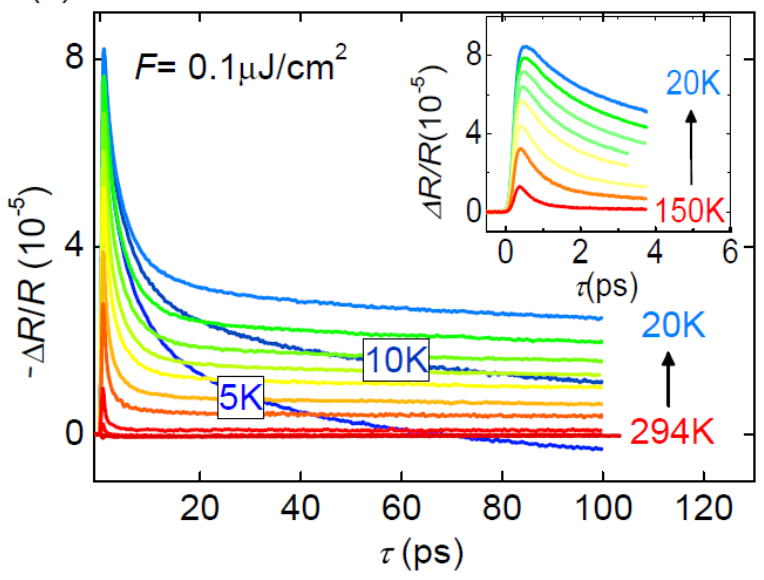

(b)

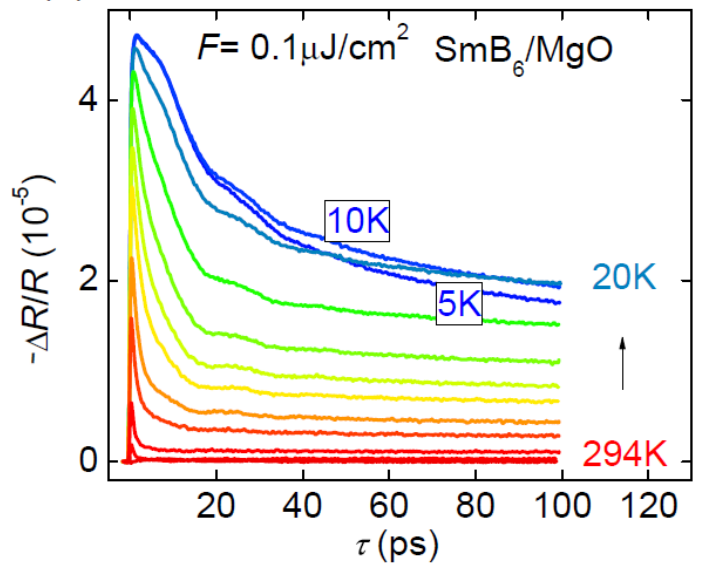

Figure 4.1: Temperature dependence of the time-resolved PI reflectivity measured in $\mathrm{SmB}_{6}$ (a) bulk single crystal and (b) thin film. A low laser fluence of $0.1 \mu \mathrm{J} / \mathrm{cm}^{2}$ is used in the measurements.

Time-resolved measurements of pump induced (PI) probe reflectivity changes $\frac{\Delta R}{R}$ at different temperatures are displayed in figure 4.1. At zero delay, $\frac{\Delta R}{R}$ rises sharply due to the hot electrons generation. Following the initial excitation, electrons quickly thermalize through electron-electron $(e-e)$ scattering and phonon emissions. During this process, energies of hot electrons experience significant relaxation and the photocarrier density is greatly amplified, which is represented by the sub-picosecond rise of the pump-probe signal close to zero delay. During the slow recovery process, both monotonic decays and coherent oscillations are observed. 
(a)

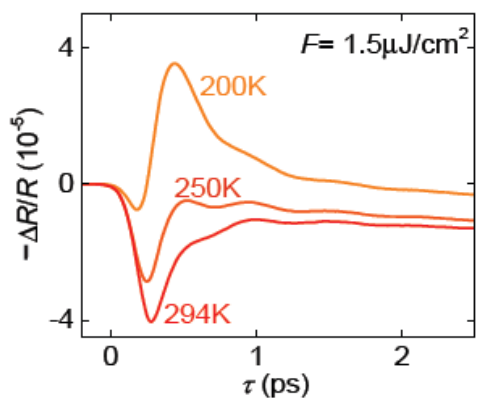

(b)

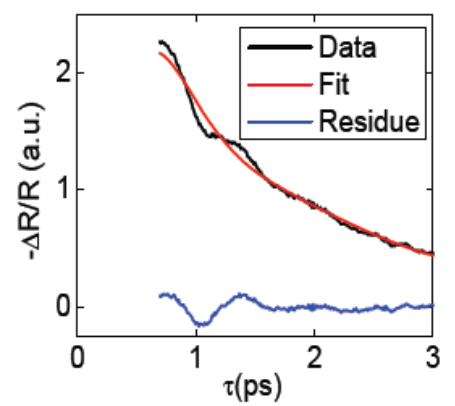

(c)
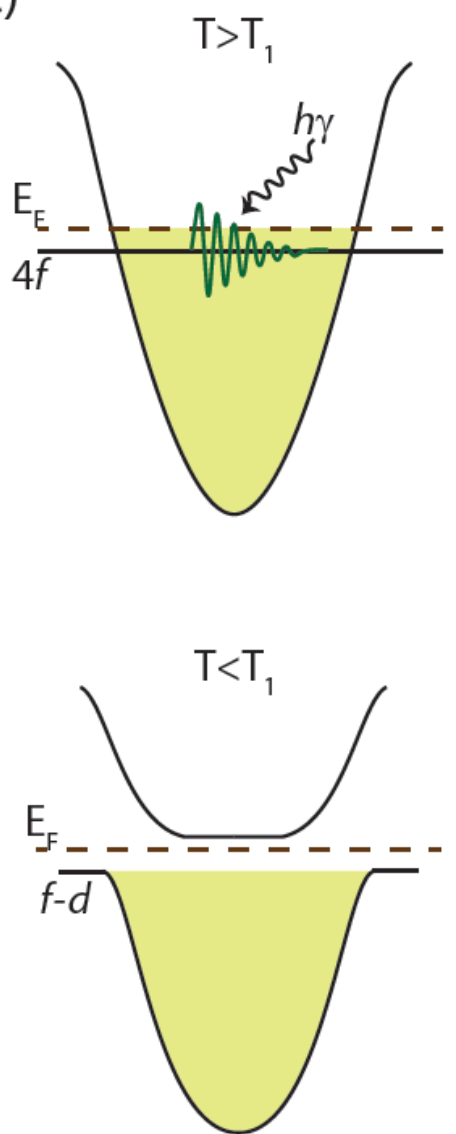

Figure 4.2: Temperature dependence of $f$-band plasmon mode (a) PI reflectivity measurement of single crystal at high temperatures, showing a $1.7 \mathrm{THz}$ oscillation signal $\left(S_{1}\right)$. (b) A plot showing the extraction of the oscillations from $\frac{\Delta R}{R}$.(d) Slower time-dependent signal is subtracted is to extract the oscillating signal which are plotted as a function of the measurement temperatures. (c) Illustration of evolvement of $S_{1}$ at hybridization gap opening

Between $300 \mathrm{~K}$ and $120 \mathrm{~K}$, a $1.7 \mathrm{THz}$ oscillation signal $S_{1}$ is observed in the single crystal (Figure.4.2 (a,b,d)). $S_{1}$ is temperature independent above $120 \mathrm{~K}$, but exhibits abrupt decoherence below $T_{1}=100 \mathrm{~K}$. Frequency of $S_{1}$ remains constant when varying the probe wavelength and the incidence angle, which indicates that $S_{1}$ corresponds to a mode almost non-dispersive at the zone center. Optical phonons fit 
such description. However, $1.7 \mathrm{THz}$ is substantially lower than all the known optical phonon branches [114-116]. Figure 4.3 shows acoustic and optical phonons in $\mathrm{SmB}_{6}$ single crystal when measured by neutron scattering.

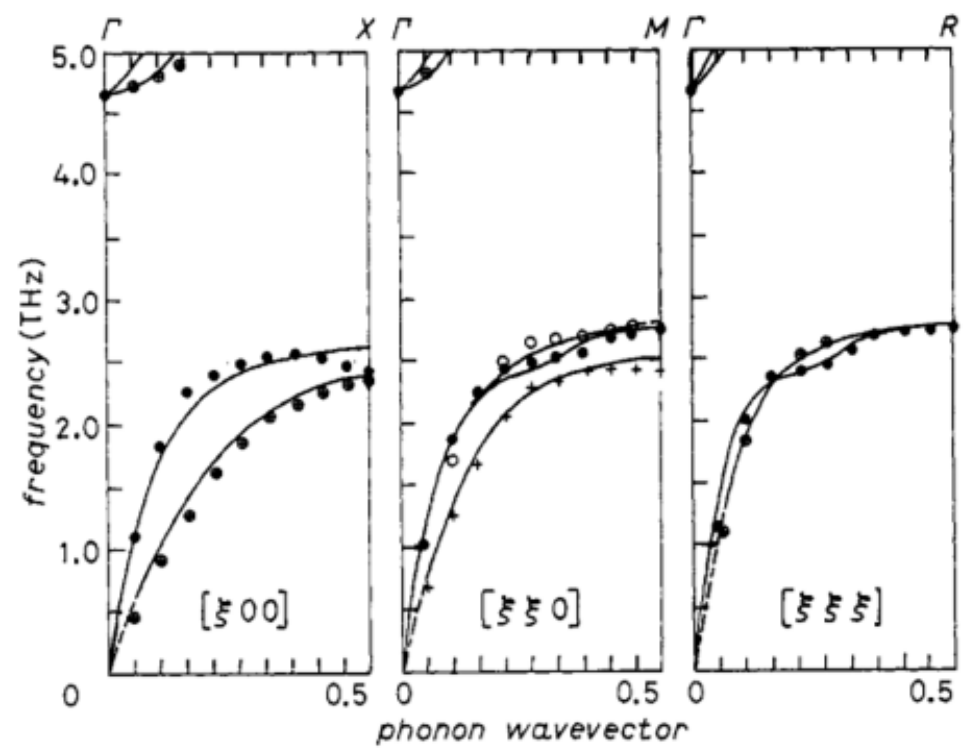

Figure 4.3: Phonons in $\mathrm{SmB}_{6}$ acquired from neutron scattering [115]. The high frequency branch $(\sim 4.5 \mathrm{THz}$ corresponds to optical phonons and the low frequency branch is the acoustic phonons)

The strong temperature dependence also rules out defect related modes. Instead, we attribute $S_{1}$ to the bulk plasmon resonance of the strongly screened $4 f$ heavy fermions (Figure 4.2(c)). $4 f$ electron plasmon frequency can be expressed as:

$$
\omega_{p}=\sqrt{\frac{n_{4 f} e^{2}}{m^{*} \epsilon_{o p} \epsilon_{0}}}
$$

Its frequency is determined by electron density $\left(n_{4 f}\right)$, effective mass $\left(m^{*}\right)$, and the effective permittivity relating to the screening by $f$ - $d$ interband transition $\left(\epsilon_{o p}\right)$. A similar frequency of this resonance $(5.3 \mathrm{meV}=1.3 \mathrm{THz})$ is reported by spectroscopy measurements where the reflectivity of the sample is measured as a function of light 
frequency [7]. We note that, $n_{4 f}$ and $\epsilon_{o p}$ both sensitively depends on the samples' chemical potentials, which may vary due to differences in synthesis conditions or surface adsorptions in ambient environments. It is very likely that the small frequency difference between our measurement and the earlier spectroscopy report is associated with such chemical potential variation. The constant frequency of $S_{1}$ above $120 \mathrm{~K}$ indicates a metal-like band structure. The drastic decoherence of $S_{1}$ signal around $T_{1}$ marks the opening of the $f$ - $d$ hybridization gap (Fig.2(c)). As temperature decreases, the heavy $f$ electrons strongly hybridize with the light $d$ electrons and opens a gap. Characteristic $f$-band plasmon thus disappears, and new electron bands form with mixed $f$ - $d$ characters. Similar signal is observed in the thin film but with a different frequency of $2.8 \mathrm{THz}$ (see figure 4.4). Defects present in the thin film can raise the chemical potential and lead to a larger electron density. In addition, the close proximity with wide bandgap $\mathrm{MgO}$ substrate is expected to further reduce the screening strength (smaller $\epsilon_{o p}$ ). Both effects are expected to give rise to a higher plasmon frequency. 


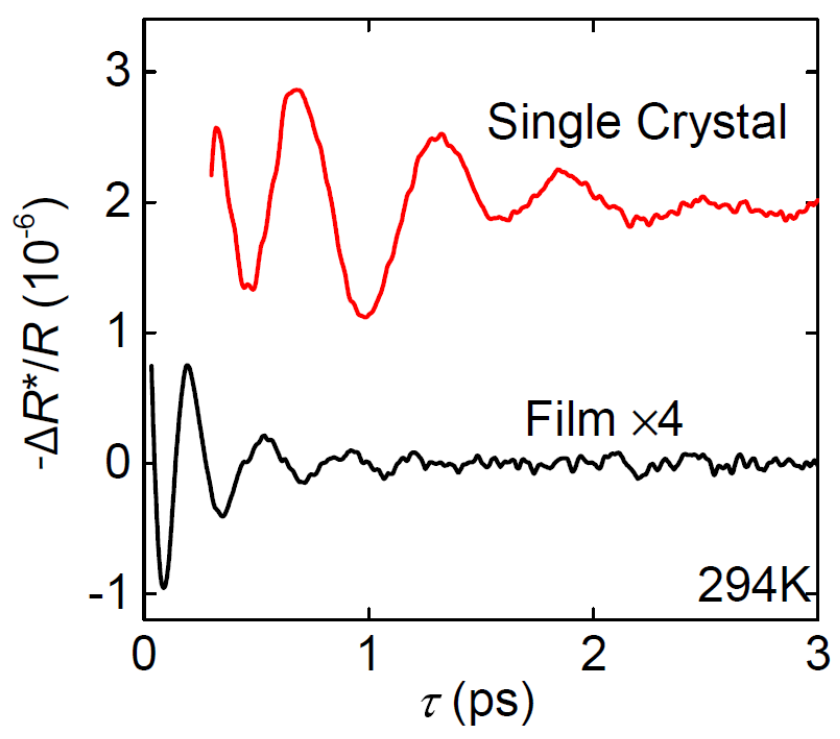

Figure 4.4: $f$-band plasmon mode in thin film compared with single crystal.

While coherent oscillation signals reveal the gap opening at $T_{1}=100 \mathrm{~K}$, monotonic decays in $\frac{\Delta R}{R}$ at low temperatures allow further characterizations of the hybridization gap. We analyze the data using a phenomenological Rothwarf-Taylor (RT) model [117], which were developed for systems with a narrow bandgap [42,44,118,119]. In these systems, zero density of states inside the gap forbids the excited electron-hole pairs from releasing energy gradually through sequential emissions of low energy phonons. While the emission of phonons with energy above the bandgap is allowed, these high energy phonons can in return re-excite new electron-hole pairs. Therefore the complete relaxation of photocarriers cannot occur before the fully decay of high energy phonons The later takes place mainly through spatial diffusion or scattering with low energy phonons. Such mechanism, often referred to as "phonon bottleneck", is often present in systems with discontinuous or discrete energy distributions [120]. At low pump fluence, maximum intensity $\left(I_{\max }\right)$ of $\frac{\Delta R}{R}$ signal represents the photocarrier density after fast $e-e$ thermalization $\left(n_{p c}\right)$, which depends strongly 
on the thermal electron distribution prior to the excitation. Described by the RT model, the temperature dependence of the thermal carrier density can be extracted from $[42,117]$ :

$$
n(T) \propto \frac{T_{\max }(T \rightarrow 0}{I_{\max }(T)-1}
$$

At low temperatures, $I_{\max }$ increases dramatically (Figure 4.1), consistent with the reduction of $n$ in presence of a hybridization gap $E_{g}[42]$ :

$$
n(T) \propto T^{1 / 2} \exp \left[\frac{-E_{g}(T)}{2 k T}\right]
$$

Assuming a gradually changing gap energy, which is supported by STS measurements $[27,28]$, we take a linear approximation of $\left.E_{(} g\right) \approx E_{g}^{*}-c T$, where $E_{g}^{*}$ is the zero temperature gap energy. Equation (4.3) becomes

$$
n(T) \propto T^{1 / 2} \exp \left[-\frac{E_{g}^{*}-c T}{2 k T}\right]=\exp \left[\frac{c}{2 k} T^{1 / 2}\right] \exp \left[-\frac{E_{g}^{*}}{2 k T}\right]
$$

In both single crystal and thin film samples, $n(T)$ agrees very well with this RT model description between $20 \mathrm{~K}$ and $100 \mathrm{~K}$ (Figure 4.5). Above $100 \mathrm{~K}$, clear deviations from the RT model are observed, which is consistent with the high temperature gap-less metallic state concluded from the plasmon measurements. Least square fitting yields $E_{g}^{*}$ of $20.8 \mathrm{meV}$ for single crystal and $14.5 \mathrm{meV}$ for thin film. In the meanwhile, the transport activation energy extracted from electrical measurements (Fig. 4.7) are considerably lower $(9.2 \mathrm{meV}$ for single crystal and $5.7 \mathrm{meV}$ for thin film). The gap energy obtained from pump-probe and transport measurements interestingly fall into the two distinct categories found in literature reports: $15 \mathrm{meV}-$ $20 \mathrm{meV}[29,31,121-124]$, and less than $10 \mathrm{meV}[24,28,124-127]$. Seeing values from the two categories simultaneously on the same samples implies that, the discrepancy 
in gap measurements cannot be simply explained by sample variations. Other intrinsic factors, such as a large temperature dependence in carrier mobility, need to be considered.

(a)

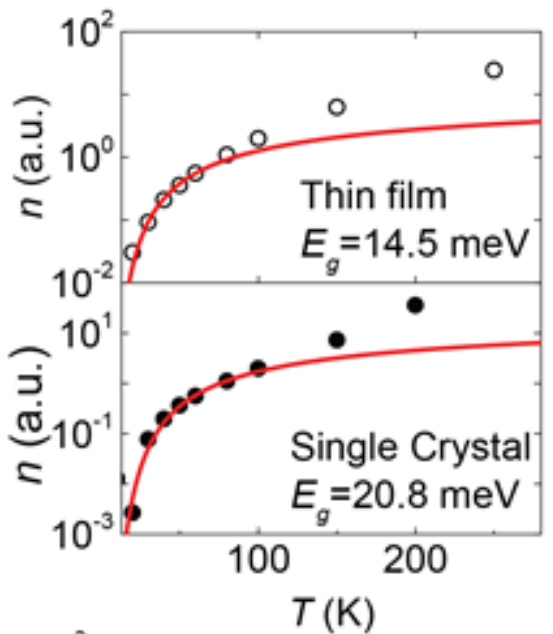

(b)

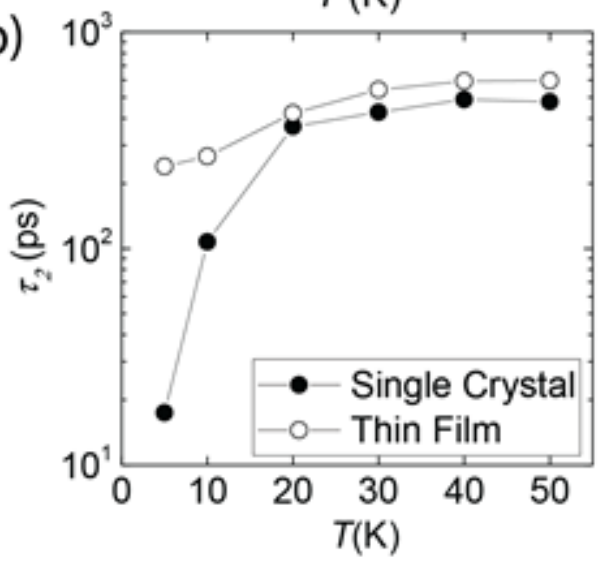

(c)
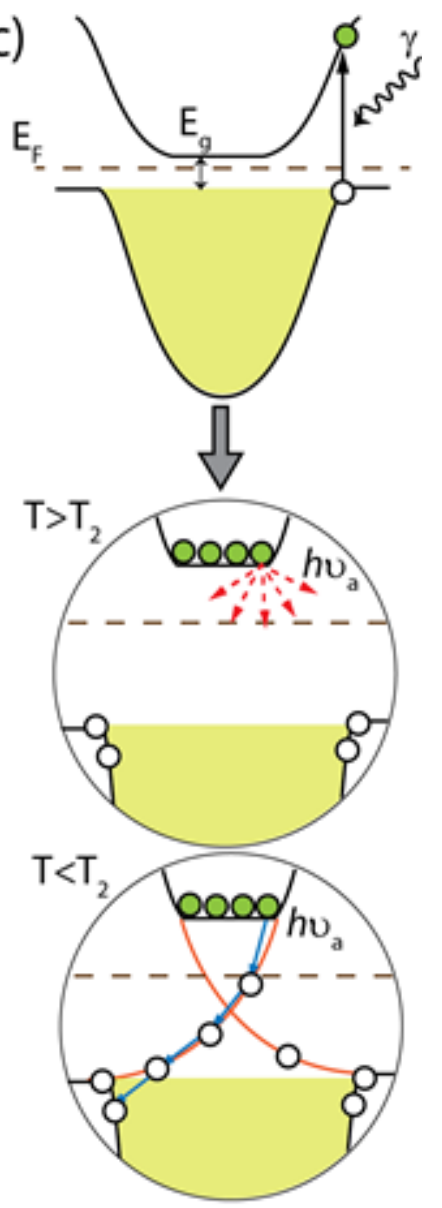

Figure 4.5: (a) Thermal carrier densities extracted from the maximum amplitudes of PI reflectivity change signals $\left(\frac{\Delta R}{R}\right)$ plotted as a function of the temperature. Fitting of $n(T) \propto T^{1 / 2} \exp \frac{-E_{g}^{*}}{2 k T}$ is performed to obtain the gap energies $E_{g}$. (b) Temperature dependence of the slow relaxation constant $\left(\tau_{2}\right)$, extracted from bi-exponential decay fitting of PI reflectivity change $\left(\frac{\Delta R}{R}\right)$. (c) Illustration of the "phonon bottleneck" phenomena induced by the narrow hybridization gap above $T_{2}=20 \mathrm{~K}$, and the surface in-gap state assisted carrier recombination process below $T_{2}$.

Temporal profile of $\frac{\Delta R}{R}$ decay can be fitted by a bi-exponential function with two decay constants and $\left(\tau_{1} \sim 10^{0} \mathrm{ps}, \tau_{2} \sim 10^{2} \mathrm{ps}\right)$. Within the frame of RT model, 
the fast decay time $\tau_{1}$ is inversely proportional to $2 n+n_{p c}[42,118]$. As the thermal carrier density $n$ decreases, the fast decay is expected to slow down. The much longer decay time $\tau_{2}$ is related to a slower relaxation process involving carrier diffusion and lattice cooling. These processes also tend to be suppressed at low temperatures. Therefore, RT model predicts a slowing carrier relaxation as temperature decreases. This is exactly what's observed between $20 \mathrm{~K}$ and $100 \mathrm{~K}$ (Figure 4.1). However, below $T_{2}=20 \mathrm{~K}$, temporal decay dramatically accelerates in the single crystal (Figure 4.1 (a)). Similar but less significant trend is also observed in the thin film (Figure 4.1(b)). Such features raise the question of whether RT model is still valid below $T_{2}$. The drastically different relaxation dynamics, however, can be explained considering the emerging of surface in-gap states (Fig. 4.5(b)). At higher temperatures, carrier relaxation is restricted by the "phonon bottleneck" caused by the zero density of states within the narrow energy gap. However, when in-gap states forms at the surface $[2,8,13,15,27,31,121]$, "phonon bottleneck" is lifted. Surface in-gap states provide effective pathways for conduction band carriers to relax their energy (after diffusing to the surface) through multi-step scattering with acoustic phonons. This surface assisted carrier relaxation mechanism is very similar to the Shockley-ReedHall (SRH) process well-known in semiconductors $[112,128]$, with the difference that typical SRH process is assisted by localized surface in-gap states instead. The carrier recombination rate (S) in SRH is determined by: $S \approx v_{T} n_{s} / n$, where $v_{T}$ is the carrier thermal diffusion velocity and $n_{s}$ is the surface state density. $\mathrm{In} \mathrm{SmB}_{6}$, the sharply increased surface state density $n_{s}$ below $20 \mathrm{~K}$ greatly enhances the recombination rate $S$ and produces the significantly different reflectivity change time profile. We note that "overshoot" pass pre-excitation value is observed in the $\frac{\Delta R}{R}$ data at 5 K. Similar phenomena is also present in SRH processes with a large $S[112,128]$. 
Lattice heating can produce a change in reflectivity that is weaker but opposite to the effects of photocarriers. When photocarriers in the bulk diffuse toward the surface and relax to the valence band through the surface state, large amount of energy is "dumped" to the lattice of the surface layer. The resultant significant surface heating can overpower the photocarrier effect, and thus explains the reflectivity signal "overshoot". It is worth noting that the above discussed effect is observed only when the pump fluence is sufficiently low (Fig. 4.9). While laser heating is expected to play an important role, other factors that can potentially impact the surface states, such as pump produced thermal stress and the photocarrier related renormalization effects, should also be considered and are worth further investigation. Carrier relaxation in the thin film sample is slower comparing to the single crystal in all temperatures. We attribute this difference to the higher defect density and associated carrier trapping. This can also lead to reduced carrier diffusion speed and cause the less significant change of relaxation dynamics observed below $20 \mathrm{~K}$. 
(a)

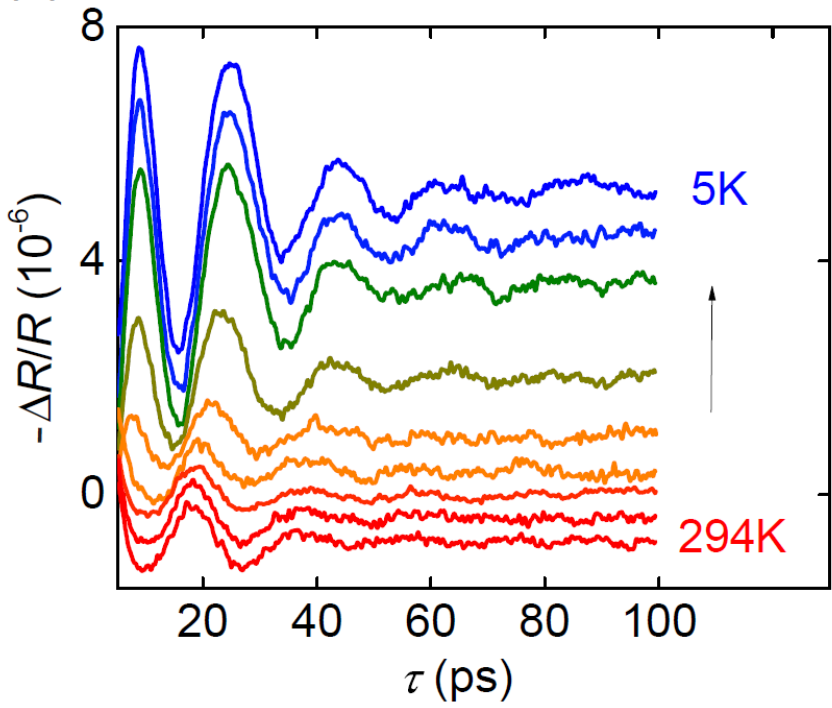

(b)

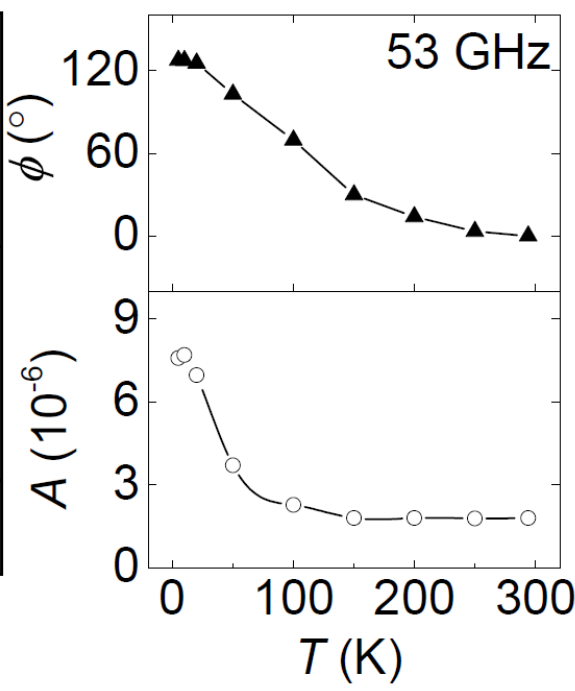

Figure 4.6: Strain wave in thin film sample (a) Temperature dependence of the 53 $\mathrm{GHz}$ strain wave oscillations extracted from the PI reflectivity measurements. (b) Amplitude of the oscillations sharply increases at $100 \mathrm{~K}$ and the phase gradually varies with the change of temperature. Pump fluence of $1.5 \mu \mathrm{J} / \mathrm{cm}^{2}$ is used in the measurements.

A damped oscillation, $S_{2}$, of much lower frequency $(53 \mathrm{GHz})$ is uniquely present in the thin film (Figure 4.1(b), Figure. 4.6(a)). We attribute $S_{2}$ to the optically excited strain wave which reflects repeatedly at the top and bottom surface of the film [129]. Surface absorption of the pump pulse induces a local thermal stress $(\sigma)$ and initializes the strain wave propagating and reflecting inside the film. The resultant periodically changing strain at the film surface transiently induces changes in the local density of states (LDOS) and surface deformation, both resulting in the modulation of measured probe pulse reflection. Considering a model where the top surface of the film is free and the $\mathrm{SmB}_{6} / \mathrm{MgO}$ interface is fixed by the substrate, the expected oscillation frequency is $f=v / 4 d$ [130], where $v$ is the speed of sound and $\mathrm{d}$ is the film thickness $(65 \mathrm{~nm})$. According to this formula, $f=53 \mathrm{GHz}$ corresponds to a sound speed of $1.4 \times 104 \mathrm{~m} / \mathrm{s}$ in $\mathrm{SmB}_{6}$, which is high but reasonable given the 
well-known hardness of borides. While the frequency remains constant throughout the measurements, phase shift of $S_{2}$ is observed upon cooling, which is owing to the variance of ratio between stress induced changes in refractive index and extinction coefficient [129]. More interestingly, amplitude of $S_{2}$ experiences a sharp upturn at $100 \mathrm{~K}$. Based on a simple isothermal model, pump induced thermal stress can be expressed as $\sigma=-3(Q / C) \beta K$, where $Q$ is the energy deposited by a single pump pulse, $\mathrm{C}$ is specific heat, $\beta$ is thermal expansion coefficient and $K$ is the bulk modulus. Since there is no evidence of discontinuous change of the involved parameters around $100 \mathrm{~K}[131,132]$. The upturn in $S_{2}$ amplitude is more likely due to the enhanced effect of stress on the local density of states at low temperatures, and possibly is related to the gap opening at $100 \mathrm{~K}$. It has been reported that uniaxial stress (application of pressure) can effectively modulate the hybridization gap size [124,133,134]. With $\mathrm{Q}=1 \times 106 \mathrm{Jm}^{-3}$ and literature reported values of $\mathrm{C}=4 \times 105 \mathrm{JK}^{-1} \mathrm{~m}_{-}^{-3}[132], \beta$ $=-5 \times 10^{-6} \mathrm{~K}^{-1}$ [131], $\mathrm{K}=147 \mathrm{GPa}$ [135], a rough estimation yields kbar at low temperatures, which is significant considering that a pressure larger than $40 \mathrm{kbar}$ can suppress the gap completely $[124,133]$. We note that the thermal stress not only generate changes in the LDOS close to Fermi levels but may also impact the higher energy states, and the overall effects contribute to the change in $800 \mathrm{~nm}$ probe's reflectivity approaching $10^{-5}$ level at a pump fluence of $1.5 \mu \mathrm{J} / \mathrm{cm}^{2}$.

Here we discuss the possible nature of the observed in-gap states. Besides of topological surface states, chemical potential shift at polar surface can also lead to metallic in-gap states [38]. As pointed out by Frantzeskakis, et al. [39], the two mechanisms can be difficult to distinguish, for example, in ARPES measurements due to the resolution limit. However, we argue that the in-gap states observed here cannot be due to the second mechanism. First, the polar surface states are often 
temperature independent, though the in-gap states we observe form only below $20 \mathrm{~K}$. Second, higher chemical potential favors the filling of polar surface states, in contrast the in-gap states we observe are destroyed at higher electron temperatures (i.e. higher effective chemical potential). Another remark is that, although analysis considering only uniform bulk properties well explains the data, such consistency doesn't preclude the possible surface nature of the in-gap states we observed. Penetration depth of 400nm pump laser in $\mathrm{SmB}_{6}$ is $d=\frac{\lambda}{4 \pi k} \approx 15 \mathrm{~nm}$ (extinction coefficient [7]). Based on the quantum oscillations observed by torque magnetometry in $\mathrm{SmB}_{6}$ single crystal [33], surface states Fermi velocities $v_{F} \sim(2.9 \pm 0.4) \times 10^{5} \mathrm{~m} / \mathrm{s}$ near $\bar{\Gamma}$ point and near $v_{F} \sim(6.5 \pm 0.21) \times 10^{5} \mathrm{~m} / \mathrm{s}$ near $\bar{X}$ point can be derived [11]. Conversion

to topological surface state penetration depth $\xi=\frac{\hbar v_{F}}{E_{g}}$ [136] yields values of 9.1 $\mathrm{nm}$ and $20 \mathrm{~nm}$, comparable to the laser penetration. Therefore, ultrafast pumpprobe measurements can be considered surface sensitive tools with regard to the investigations of surface states in $\mathrm{SmB}_{6}$.

\subsection{Conclusion}

By studying the transient carrier dynamics in $\mathrm{SmB}_{6}$, a coherent picture of the band structure evolvement in $\mathrm{SmB}_{6}$ is successfully derived. We found two characteristic temperatures $T_{1}$ and $T_{2} . T_{1}=100 \mathrm{~K}$, corresponding to the opening of $f-d$ hybridization gap, is pin-pointed by the abrupt disappearance of $f$-band plasmon. Below $20 \mathrm{~K}$, dramatically accelerated carrier relaxation as well as significant surface lattice heating are detected. These phenomenon can be well explained by the emergence of surface in-gap state and the resultant surface assisted carrier recombination process. Measurement results obtained in single crystal and thin film samples qualitatively agree with each other, supporting the existence of a possible surface 
conducting layer at low temperature.

More studies are necessary to conclude whether the observed in-gap states are indeed topological surface states. For example, it will be very interesting to look for plasmon modes associated with these in-gap states. The plasmon dispersion relation and damping properties can provide highly valuable insight into the in-gap states' dispersion and their correlations with the bulk. Also, time resolved measurements of the spin dynamics can also be performed to evaluate the spin-orbit coupling effect and other spin related features predicted by TKI theory. 


\subsection{Supplementary Information}

\subsubsection{Transport Measurements}

(a)

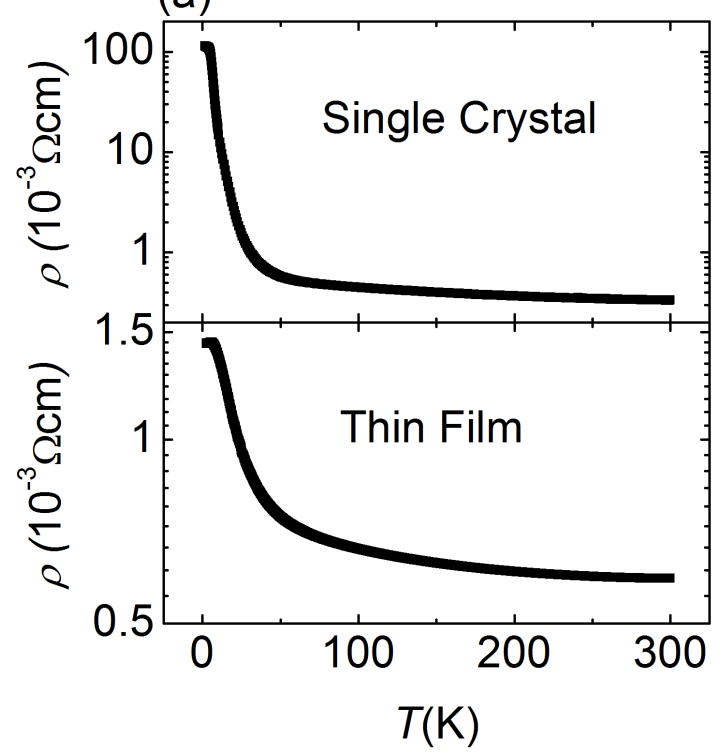

(b)

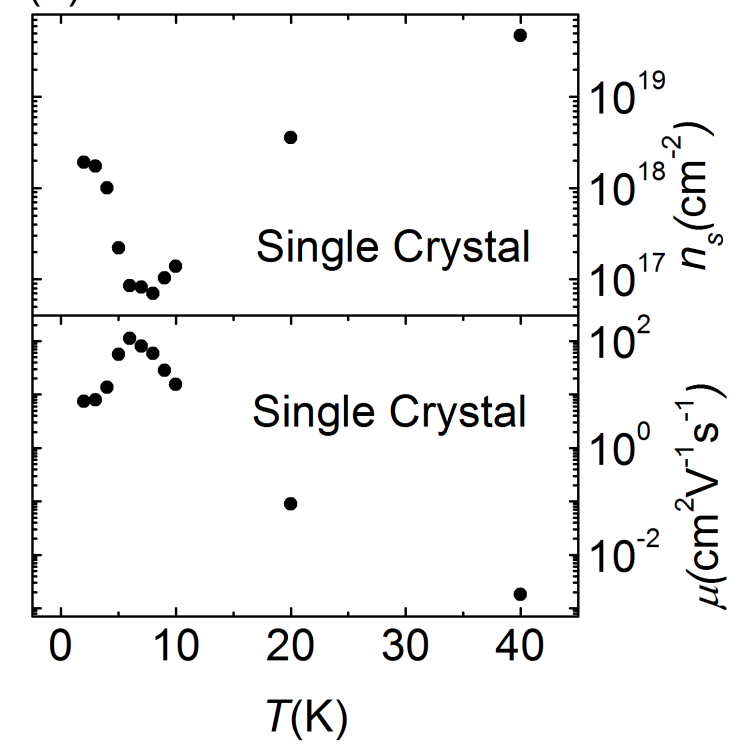

Figure 4.7: Resistivity vs temperature for both (a) single crystal and thin film. Resistivity measurement for the single crystal was done in hall bar configuration and thin film was done in van der pauw configuration. The resistivity saturates at temperatures below $4 \mathrm{~K}$, consistent with the existence of surface conducting states. (b) Sheet carrier density and mobility as a function of temperature for the single crystal extracted from the hall voltage measurement. 


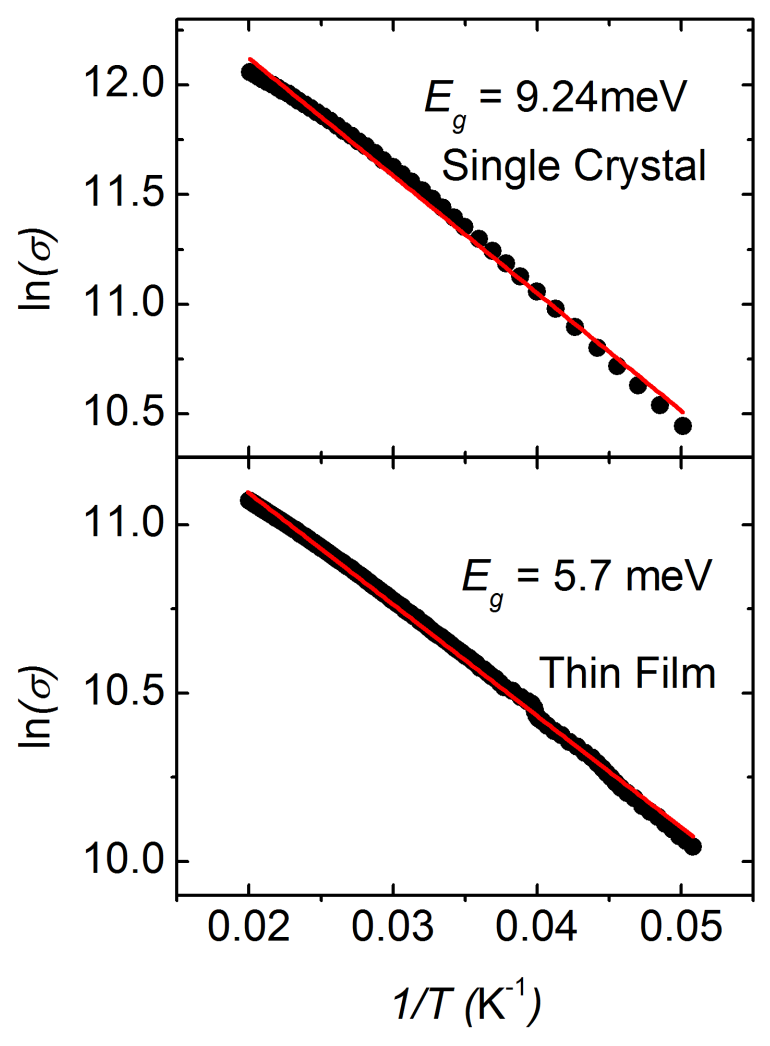

Figure 4.8: $\ln (\sigma)$ vs. $1 / T, 20 \mathrm{~K}<\mathrm{T}<50 \mathrm{~K}$, for single crystal (on the top) and thin film (on the bottom), where $\sigma$ is conductivity. The fit, shown in red, is done using thermal activation law equation given by $\sigma(T)=\sigma_{0} \exp \left(-E_{g} / 2 k T\right)$. Where, $k$ is the Boltzmann constant, and $E_{g}$ is the energy gap 


\subsubsection{Fluence Dependence}

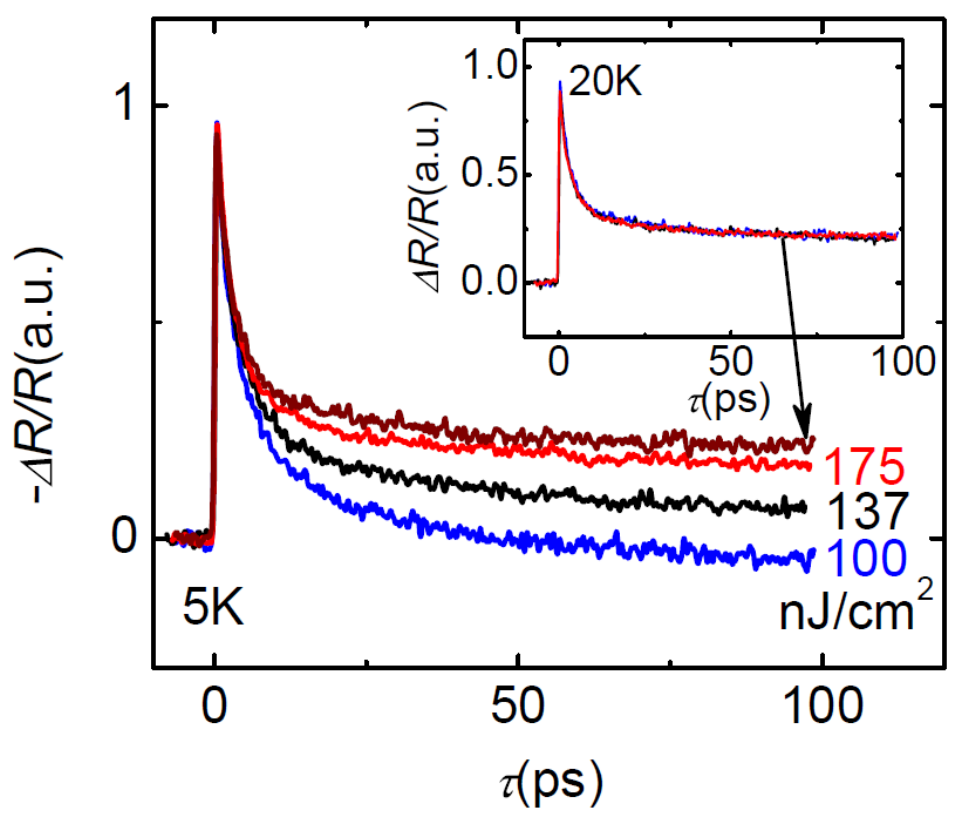

Figure 4.9: Pump induced reflectivity measurement for single crystal at $5 \mathrm{~K}$ as a function of the pump fluence. The signal amplitude is normalized to see the relaxation dynamics. Inset to the figure shows the change in reflectivity as a function of pump fluence at $20 \mathrm{~K}$. 


\section{Chapter 5}

\section{Optical helicity control of surface current in $\mathrm{SmB}_{6}$}

\subsection{Abstract}

$\mathrm{SmB}_{6}$ is suggested to be a promising candidate for topological Kondo insulator. Transport measurements and spin resolved ARPES measurements have shown topologically protected surface state and the helical spin texture with spin-momentum lockage. Here, we report current injection in $\mathrm{SmB}_{6}$ thin film with circularly polarized light at oblique incidence, an outcome of momentum-spin lockage. A polarizationindependent photovoltage was also detected. Both signals exhibited strong temperature dependences. While the polarization-independent photovoltage is likely due to thermoelectric or photovoltaic effects, the circular photogalvanic effect is due to topological surface states. To shed more light onto the nature of the surface states observed in $\mathrm{SmB}_{6}$, experiments were performed on thin films with different thickness. We report spin polarized photocurrent, direct result of spin momentum lockage, in $\mathrm{SmB}_{6}$ for the first time. 


\subsection{Introduction}

Topological insulators (TI) are materials with insulating bulk and conducting surface state (SS). The SS has many interesting properties such as: time reversal symmetry, topological protection, and spin-momentum lockage due to a special electronic structure [137]. Since the spin and momentum are locked perpendicular, on the surface plane, manipulating electron momentum can manipulate spin direction and vice versa. Hence, TI might be a key to the further development in the field of spintronics, where the challenge is generation and control of spin current [138]. Moreover, when a true TI and a superconductor are brought into a close contact, the topological surface can be made superconducting, which results in creation of Majorana fermions $[22,23]$. The creation of Majorana fermions using topological insulator would be a significant breakthrough in physics. Recently in $\mathrm{Bi}_{2} \mathrm{Se}_{3}$, a tra-

ditional TI, spin polarized photocurrent dependence on the helicity of incident light has been theorized and experimentally reported $[52,53]$. Unlike traditional TI materials, in which surface transport is dominated by the bulk transport, $\mathrm{SmB}_{6}$ seems to possess a true insulating bulk [24] which will make it even more interesting for applications. In a TI, the coupling of light can result in dc transport that is sensitive to the helicity (right versus left-circular polarization) of the incident light. However in $\mathrm{SmB}_{6}$, no spin polarized photocurrent has been reported so far. In this paper, we report helicity control of surface current, a prominent feature of a true TI. Photons in Circularly Polarized (CP) light have a well-defined angular momentum, CP light can couple to the spin of the surface electrons in surfaces with broken rotational symmetry. Experimentally, the rotational symmetry of a surface can be broken by an obliquely incidental (OI) light. In this paper, we report that the illumination of $\mathrm{SmB}_{6}$ with $\mathrm{CP}$ OI light generates a photovoltage (PV) and reversal of the CP light 
switches the polarity of the PV. To explain the result, we propose that the change in direction of PV with the change in the helicity of the light, also called circular photogalvonic effect (CPGE), is due to the topological helical Dirac fermions on the surface of the $\mathrm{SmB}_{6}$ and can not be due to Rashba type spin-orbit coupling as a result of band bending or dangling bonds common in this system.

\subsection{Experimental Setup}

Thin films of $\mathrm{SmB}_{6}$ are grown on top of $\mathrm{MgO}$ substrates by pulsed laser deposition (PLD).These samples are acquired from Yanjun Ma, Chang-Beom Eom(University of Wisconsin, Madison). X-ray diffraction (XRD), right after the growth, reveals polycrystalline nature of the film. On these samples, Ti/Au contacts are made with photolithography followed by e-beam evaporation in cleanroom environment. For the experiments, $1.8 \mathrm{~mW}$ of $800 \mathrm{~nm}$ femtosecond pulsed laser is focused to $30 \mu \mathrm{m}$ on the sample surface with $30^{\circ}$ with respect to the $\mathrm{z}$ axis. Sample temperature is varied using a close cycle cryostat to measure the PV as a function of temperature. 


\subsection{Results and Discussions}
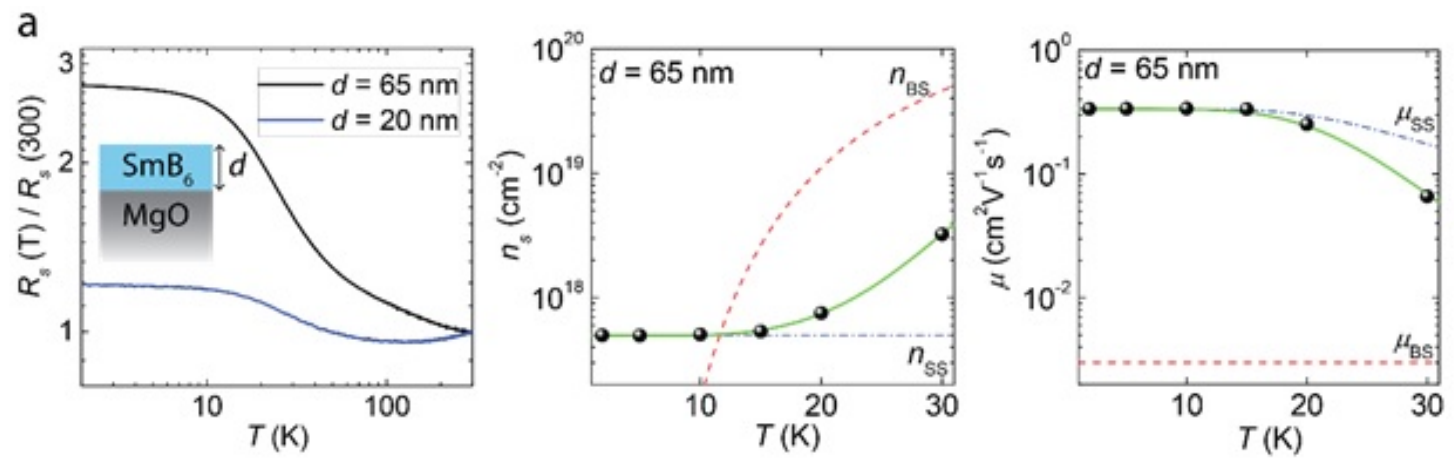

Figure 5.1: Transport properties of $\mathrm{SmB}_{6}$ thin films (a) Temperature dependences of sheet resistances measured in $65 \mathrm{~nm}$ and $20 \mathrm{~nm}$ thick films. Data are normalized by their values at $300 \mathrm{~K}$. (a,b) Temperature dependent sheet carrier density and mobility measured in a $65 \mathrm{~nm}$ film (scattered plot). Lines indicate a possible fitting considering parallel conductions from the surface and bulk. Red and blue dash lines show the separate contributions from bulk states $\left(n_{B S}, \mu_{B S}\right)$ and surface states $\left(n_{S S}, \mu_{S S}\right)$, respectively. Green solid lines show the net fitting results.

Low temperature resistance plateaus, presumably associated with the surface states, were observed in all the thin film samples measured (Fig. 5.1a). Transport model based on two parallel carrier types $\left(n_{s}=\frac{\left(n_{B S} \mu_{B S}+n_{S S} \mu_{S S}\right)^{2}}{n_{B S} \mu_{B S}^{2}+n_{S S} \mu_{S S}^{2}}, \mu=\frac{n_{B S} \mu_{B S}^{2}+n_{S S} \mu_{S S}^{2}}{n_{B S} \mu_{B S}+n_{S S} \mu_{S S}}\right.$ ) can well fit the sheet carrier density measured by the Hall effect at low temperatures (Fig. 5.1b,c). As the bulk thermal carrier density decreases $\left(n_{B S} \propto \exp \left(-E_{g} / 2 k_{B} T\right)\right.$, where $E_{g}=14.5 \mathrm{meV}$ is the gap energy), transport properties of the film below $15 \mathrm{~K}$ are dominated by the surface state. In thinner films, the relative ratio between the plateau resistance (surface state dominant) and room temperature resistance (bulk dominant) becomes smaller (Fig. 5.11a). This is consistent with the fact that thinner films have increased bulk resistances. 

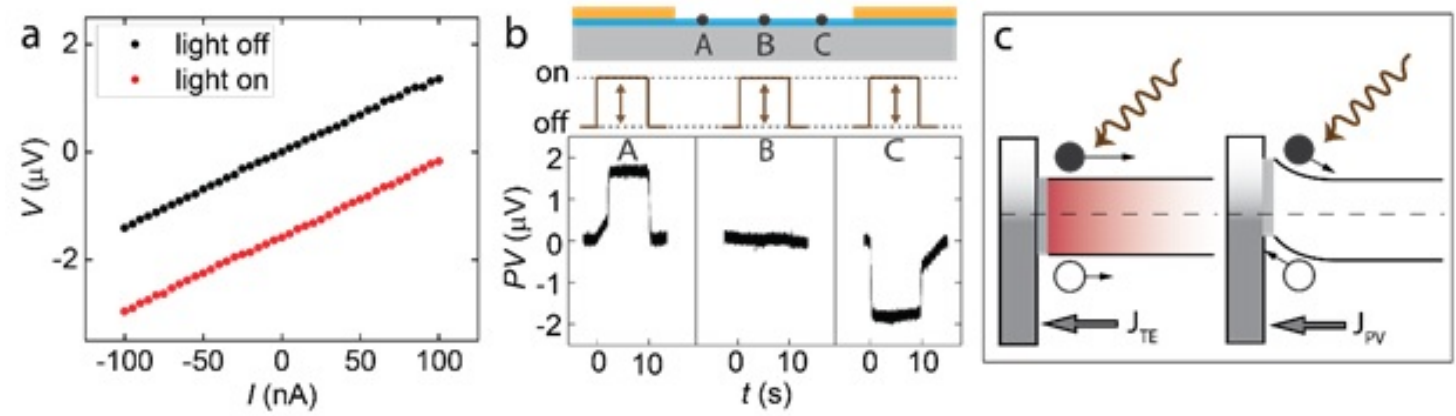

Figure 5.2: Photovoltage (PV) generated by linearly polarized light (a) I-V characteristic measured with or without the laser illumination. (b) Transient PV responses measured when linearly polarized light is focused at different positions (A, B and C) between the two voltage sensing electrodes. (c) Illustrations of the two possible mechanisms responsible for the polarization insensitive PV observed: thermoelectric effect (left) and photovoltaic effect (right).

We first discuss the low temperatures PV measurements in $65 \mathrm{~nm}$ films. Excitation wavelengths in visible and near infrared range showed very similar PV responses. This is not surprising given that the photon energies tested were all much larger than the band gap of $\mathrm{SmB}_{6}(\sim 10 \mathrm{meV})$. Data presented here were acquired using $800 \mathrm{~nm}$ laser illuminations. Figure 1a shows the four probe I-V characteristics measured at $5 \mathrm{~K}$ when linearly polarized light is illuminating between the two voltage sensing electrodes ("on") or being blocked ("off"). For a light intensity of $28 \mathrm{~W} / \mathrm{cm}^{2}$, a photovoltage offset about $2 \mu \mathrm{V}$ can be clearly measured. The constant slopes of the I-V curves indicate no measurable photoconductance change, which confirms that the light intensity used in the measurements can be considered as a weak perturbation to the material. Figure 5.2b shows the open circuit PV signal detected when the linearly polarized light is focused at three different positions (A, B, and C) relative to the voltage sensing electrodes. To study the transient photovoltage response and also to exclude the measurement artifacts from the background potential drift, the incident light is modulated by a square wave with frequency of $0.05 \mathrm{~Hz}$. Sharp rising 
and falling edges ( 10ms) of PV in response to the illumination square wave as well as several second level slow tails were both observed. When laser is focused closer to the measurement anode (position A), a positive PV response is observed. When laser is focused closer to the cathode (position C), PV signal changes into negative. At the center (position B), when the laser focal spot has approximately equal distances to the two electrodes, PV generated by linearly polarized light becomes zero. Two mechanisms that can explain the position dependent PV signals observed are illustrated in figure 5.2c. First is the thermoelectric effect (Fig. 5.2c left), where the laser induced temperature gradient and the thermal motion of carriers give rise to a thermoelectric voltage. The electromotive force generated by thermoelectric effect is proportional to the temperature gradient as well as the Seebeck coefficient of the materials: $E_{T E}=-S \nabla T . \mathrm{SmB}_{6}$ has a negative Seebeck coefficient at low temperatures $[132,139]$, therefore the hotter region is expected to have a higher thermoelectric potential, consistent with our observation. The as large as $\sim 800 \mu \mathrm{V} / \mathrm{K}$ value of the Seebeck coefficient also explains the microvolt level thermoelectric voltage generated by the weak laser excitation $[132,139]$. In the meanwhile, photovoltaic effect (Fig. $5.2 \mathrm{c}$, right) due to band bending at the interface with metal contacts ( $\mathrm{Ti} / \mathrm{Au})$ can also be present. In this case, the position dependent polarity of PV observed in our experiments will correspond to an upward band bending toward the metal contacts. Both effects can be generated by light of any polarizations or incidence directions, which is consistent with our measurements when light is focused at positions A and C. 

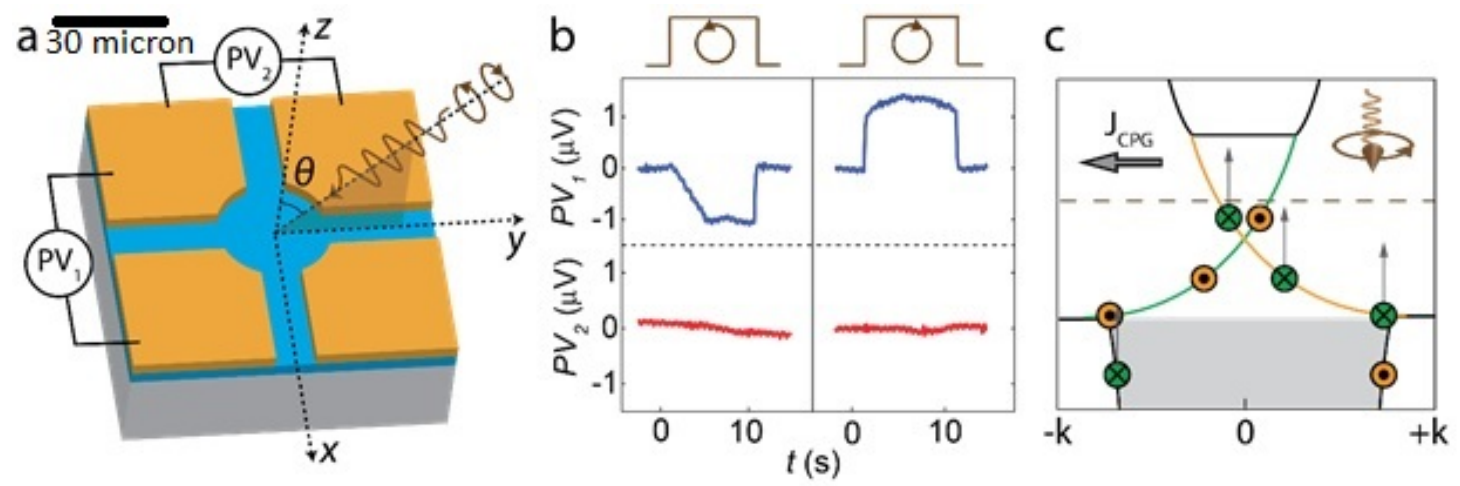

Figure 5.3: Circular photogalvanic effects (CPGE) in $\mathrm{SmB}_{6}$ thin film (a) Illustration of the measurement setup using quadrant electrodes and an oblique laser incidence angle $\left(\theta=30^{\circ}\right)$. In this measurement, circularly polarized light was focused at the center of the electrode pattern where the polarization insensitive PV signal minimizes. (b) Transient PV response to either left circularly polarized light (LCR) or right circularly polarized light (RCP) measured across two orthogonal electrode pairs. CPG effects were only observed between the electrode pairs perpendicular to the laser incidence direction. (c) Illustration of the band structure of in-gap topological surface states in $\mathrm{SmB}_{6}$ as well as the associating spin helicity. Circles with crosses and dots indicate states with opposite in-plane spin polarizations perpendicular to their orbital momentums. Obliquely incident circularly polarized light generates CPG effect by exciting surface carriers with a selected in-plane spin polarization. This local spin polarization is then converted to a k-asymmetry by the spin-momentum lockage and therefore induces a measurable photovoltage.

In contrast, when the light in focused at position B, PV measured becomes highly correlated with the polarization and incidence angle of light. To understand the correlations, we performed PV measurements at an oblique laser incidence angle $(\theta)$ of 30 degree using a quadrant electrode layout as illustrated in figure 5.3 a. Using this configuration, light induced potential gradients in different in-plane orientations can be simultaneously captured by measuring the voltages across different electrode pairs. $P V_{1}$ denotes the voltage measured across an electrode pair that were displaced with respect to each other in the direction perpendicular with the incident laser (and therefore perpendicular with the photon momentum). And $P V_{2}$ denotes the voltage measured across an orthogonal electrode pair. In this experiment, laser is focused at 
the center of the electrode pattern. At this position, potentials at the two voltage sensing electrodes generated by either thermoelectric effect or photovoltaic effect are supposed to be equal. And indeed, and are both found zero when using linearly polarized light. When circularly polarized light is used instead, large signal emerges in $P V_{1}$, while $P V_{2}$ remains zero (Fig. 5.3b). In addition, $P V_{1}$ exhibits opposite polarities for left (LCP) and right (RCP) circularly polarized light (Fig. 5.2b).

This PV signal, generated in the direction transverse to the photon momentum and controlled by optical helicity, highly resembles the circular photogalvanic effects (CPGE) observed in material systems with carrier momentum dependent spin polarizations $[52,140-146]$. Such systems include semiconducting materials and heterostructures that lack inversion symmetry $[142,146]$ and topological insulators $[53,132,140]$. In the case of topological surface states, carrier spin and orbital momentum $(\vec{k})$ are always orthogonal with respect to each other and form an in-plane helical configuration (Fig. 5.3c). When circularly polarized photons with momentum $\vec{k}_{\gamma}$ are absorbed, the transfer of spin angular momentum injects an optical helicity determined spin polarization either parallel or antiparallel to $\vec{k}_{\gamma}$. In this process, electrons with $\vec{k}_{\gamma}$ direction spin projection opposite to photons are selectively excited to states with flipped spin (Fig. 5.3 c). Due to the orthogonal relation between the spin and momentum, selective excitation of electrons of spin along $\vec{k}_{\gamma}$ also break the band's $k$ symmetry in the direction transverse to $\vec{k}_{\gamma}$. Consequently, the drift of carriers produces a CPGE photovoltage. In the case of regular semiconducting systems lacking inversion symmetry, spin-orbit coupling (SOI) is allowed. SOI can lift the spin degeneracy and form subbands with $k$ dependent spin distributions. Excitation of these subbands by circularly polarized light can also give rise to CPGE. 
$\mathrm{SmB}_{6}$ has a cubic lattice structure. The inversion symmetry of the lattice prohibits spin-orbit coupling in the bulk. Considering the large film thickness $(65 \mathrm{~nm})$ and heavy carrier mass in $\mathrm{SmB}_{6}\left(m^{*} \approx 1000 m_{0}\right)$, film confinement induced quantum well states are also unlikely to form. Therefore, the CPGE observed in our experiments can only come from the surface. Two types of possible surface states need to be considered: topological surface states, and regular surface states with a Rashba type spin-orbit coupling. Surface states in the second category can originate from the Boron dangling bonds $[34,35]$ or band bending at the polar surface $[37,38]$. We note that regular and topological surfaces states don’t necessarily exclude each other. In some materials, such as $\mathrm{Bi}_{2} \mathrm{Se}_{3}$, both states are present at the surface $[147,148]$.

In $\mathrm{SmB}_{6}$, one distinction between topological and regular surface states is that their penetration depths into the bulk are very different. The high surface carrier density measured in $\mathrm{SmB}_{6}$ at low temperatures (Fig. 5.1b) indicates a very strong carrier screening at the surfaces. The corresponding Thomas-Fermi screening length $\lambda_{T F}=\pi^{2 / 3} \sqrt{\epsilon \hbar /\left(e^{2} m^{*} n^{1 / 3}\right)}$ is below $1 \mathrm{~nm}$. Therefore, either states associated with surface dangling bonds or quantum well states formed from surface band bending are expected to be strongly confined at the surfaces within a sub-nanometer length scale $\left(\xi_{R}\right)$. In contrast, the penetration depth of topological surface states $\left(\xi_{T}\right)$ behaves differently. In many other well studied TI materials, $\xi_{T}$ is also very short. For example, $\xi$ in $\mathrm{Bi}_{2} \mathrm{Se}_{3}$ is only around $1 \mathrm{~nm}[148,149]$. However, $\xi_{T}$ can be much longer in $\mathrm{SmB}_{6}$. Based on the quantum oscillations observed by torque magnetometry in $\mathrm{SmB}_{6}$ single crystal [33], surface states Fermi velocities $v_{F} \sim(2.9 \pm 0.4) \times 10^{5} \mathrm{~m} / \mathrm{s}$ near $\Gamma$ point can be derived [20]. Bandgap energy of the thin film was previously measured to be $14.5 \mathrm{meV}$. Using these numbers, the topological surface state penetration depth can be estimated by $\xi_{T}=\hbar v_{F} / E_{g} \approx 13 \mathrm{~nm}[136]$. 
The drastically different penetration depths of topological and regular surface states in $\mathrm{SmB}_{6}$ provide an opportunity to distinguish them by investigating films with thicknesses $d$ in the range of $2 \xi_{R}<d<2 \xi_{T}$. For this purpose, we now discuss the PV measurements done in $20 \mathrm{~nm}$ films. In these thinner films, one more length scale that needs to be considered is the laser penetration depth $(\delta) .800 \mathrm{~nm}$ extinction coefficient of $\kappa \approx 2[7]$ corresponds to a laser penetration depth of $\delta=\lambda / 4 \pi \kappa \approx 30$ nm. Therefore, in these thinner films, laser will excite both the top and bottom surfaces. Though the light intensity at the top surface will be at least twice as strong as the intensity at the bottom surface. Focusing position dependent PV signals generated by linearly polarized light in $20 \mathrm{~nm}$ films were largely unchanged comparing to what were measured in $65 \mathrm{~nm}$ films. However, measured by the identical configuration as shown in figure 5.3 a, the CPGE voltages generated by circularly polarized light were no longer present. Even after the intensity of laser was increased by five times, the signal was still not detectable (Fig. 5.4a). 

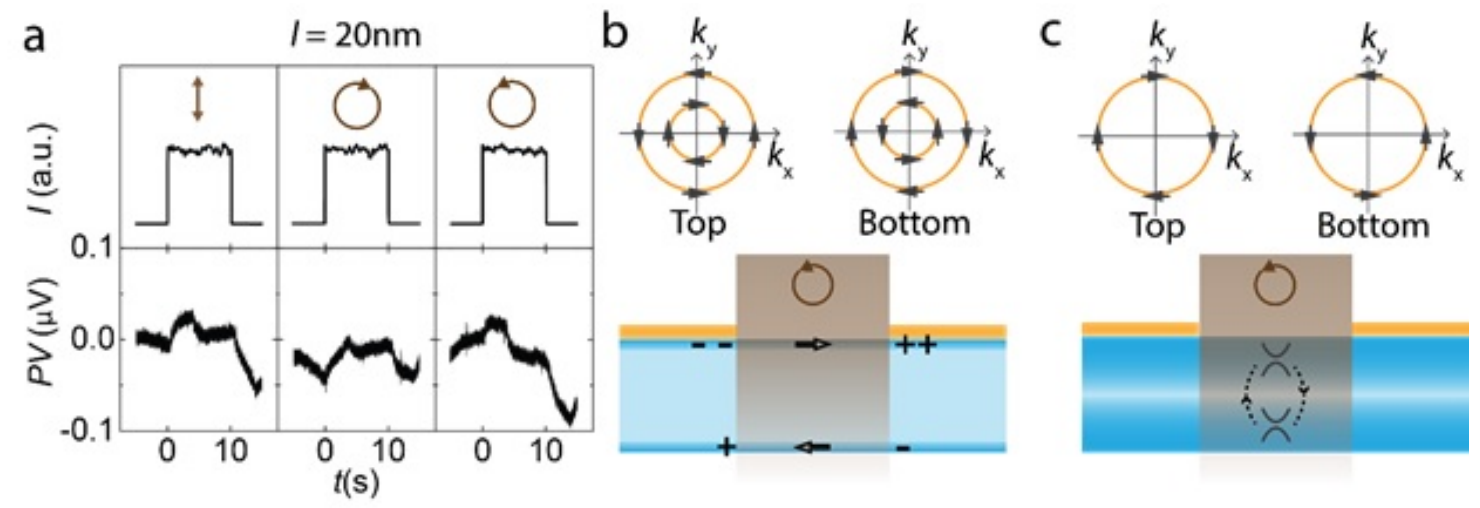

Figure 5.4: Suppression of CPGE voltage in $20 \mathrm{~nm}$ films films (a) Measured by an identical configuration as illustrated in figure 3, no CPGE was found in $20 \mathrm{~nm} \mathrm{SmB} 6$ films. (b and c, top) $k$-dependent spin configurations of regular surface states with Rashba type spin-orbit coupling (b) and topological surface state (c). (b and c, bottom) CPGE voltage measured by electrodes deposited at the top surface can be diminished when inter-surface coupling occurs. In $20 \mathrm{~nm}$ films, such scenario can only be satisfied by topological surface state (c), which has a penetration depth around $13 \mathrm{~nm}$. Regular surface states (b) originated from dangling bonds and surface band bending are expected to be much more confined at the surfaces.

Now the question is that, between topological surface states and regular surface states with Rashba type SOI, which one will generate a CPGE voltage that can be completely suppressed by reducing the film thickness to $20 \mathrm{~nm}$. In the case of regular surface states, states at the top and bottom surfaces have opposite spin helicities and are well separated by the bulk (Fig. 5.4b). One might argue that the opposite CPGE generated at the two surfaces might eventually cancel each other. However, because the light intensity decays significantly when it penetrates through the film, much stronger CPGE at the top surface is expected. Also because of the large dielectric constant of $\mathrm{SmB}_{6}$ at low temperatures $(\epsilon=1500)$ [7], the spatial charge separation induced by CPGE at the bottom surface will be further screened when the electrical potential difference is measured at the top surface. Moreover, Boron dangling bonds and surface band bending are both highly sensitive to the surface termination or 
oxygen exposure [35]. It is not clear whether such states are still present at the bottom surface (interface between $\mathrm{SmB}_{6}$ and $\mathrm{MgO}$ ). Even if they do exist at the interface, the related band characteristics as well as the Rashba spin-orbit coupling strength are expected to be very different comparing to the top surface. Due to these asymmetries discussed, while reduction of the signal is possible in thinner films, the observed complete suppression of the CPGE voltage is very unlikely to happen when considering regular surface states alone.

Instead, the very long penetration depth of topological surface states in $\mathrm{SmB}_{6}$ produces a distinctive scenario (Fig. 5.4c). Since, inter surface coupling between the top and bottom topological states can be quite significant $[148,150,151]$. Technically, the carrier exchanges between the two surfaces with opposite spin helicities can effectively "short" the voltage-sensing electrodes from the optically excited regions. And more fundamentally, scattering between the originally gap-less states at the top and bottom surfaces can induce a gap opening $[150,151]$. As a result, the momentum dependent spin configuration, which is the very reason why CPGE is generated in the first place, can also be altered $[150,151]$. These effects are highly consistent with the observed disappearance of CPGE voltages in $20 \mathrm{~nm}$ films. Thus, the CPGE observed in our experiments is likely generated from topological surface states instead of regular surface states. 

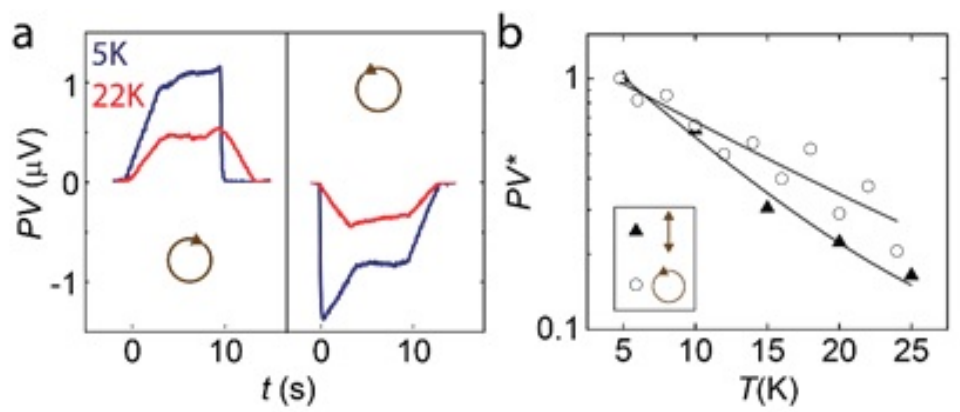

Figure 5.5: Temperature dependences of PVs in $\mathrm{SmB}_{6}$ thin film (a) Transient CPG photovoltage measured at $5 \mathrm{~K}$ and $22 \mathrm{~K}$. (a) Transient CPG photovoltage measured at $5 \mathrm{~K}$ and $22 \mathrm{~K}$. (b) Temperature dependences of both the CPG photovoltage (hollow circle) and the polarization insensitive PV signal (solid triangle). Data are normalized by their values at $5 \mathrm{~K}$. Solid lines represent exponential fittings.

The PV generated with both (linearly and circularly polarized) light decreases with the increase in temperature (see figure 5.5ab). However, the PV generated with linearly polarized light decreases faster than PV with circularly polarized light. Earlier we had argued that the PV due to linearly polarized light could be due to thermal effect. Hence, the faster decay of PV due to linearly polarized light is consistent with this argument.

\subsection{Conclusions}

From the CPGE measurement, helicity dependent photovoltage is observed in 65 $\mathrm{nm}$ thick $\mathrm{SmB}_{6}$ film grown on $\mathrm{MgO}$. CPGE measurements on this sample provided two possibilities for the origination; topological helical Dirac fermions on the surface of the $\mathrm{SmB}_{6}$ and simple outcome of Rashba type spin-orbit coupling from a regular surface due to dangling bonds. In case of topological surface state, carrier spin and momentum are always orthogonal to one another. When circular polarized light is absorbed, the spin angular momentum of the light is transferred to the surface 
electron spin. Depending on the helicity of the light, electron with certain spin can be selectively excited. Consequently, the drift of the carriers produces CPGE. Measurement on thinner sample revealed that CPGE is absent. Absence of CPGE effect in thinner film leads to conclusion that the CPGE effect is due to the presence of topological surface state and not due to regular surface. In future it will be very interesting to measure the time resolved spin dynamics with ultrafast magneto-optics Kerr effect. 


\section{Chapter 6}

\section{Role of surface water adsorptions in $\mathrm{LaAlO}_{3} / \mathrm{SrTiO}_{3}$ interfaces}

\subsection{Abstract}

Here we report the manifold effects of surface water layers on the $\mathrm{LaAlO}_{3} / \mathrm{SrTiO}_{3}$ interface properties. The spontaneous dissociation of water on $\mathrm{LaAlO}_{3}$ surface is systematically studied by density functional theory and experimental surface characterizations. Extrinsic effects from surface adsorbates were often ignored in the previous studies of the two dimensional electron gas (2DEG) formed at $\mathrm{LaAlO}_{3} / \mathrm{SrTiO}_{3}$ interfaces. However, we found that the dissociated water molecules, especially the surface protons, strongly affect the interface density of states, electron distributions and lattice distortions. Our investigations also reveal the importance of additional molecular water layers. These layers, through hydrogen bonds, provide an energetically feasible pathway for manipulating the surface-bonded protons and thus the interface electrical characteristics. 


\subsection{Introduction}

Surface properties can significantly affect the performance of semiconducting electronics [75-77]. On one hand, surface states can induce undesired carrier trapping $[78,79]$. On the other hand, surface could assist in doping for many material systems where bulk doping is restrained. Such systems include wide band gap semiconductors (e.g. diamond) [80,81], two dimensional materials (e.g. graphene) [82,83], nanostructures [76,84], and chemically fragile organic semiconductors [85].

$\mathrm{LaAlO}_{3} / \mathrm{SrTiO}_{3}$ heterostructure is one system where the surface related effects are vital $[1,66,152,153]$. In this system, the formation of interfacial two dimensional electron gas (2DEG) $[1,64,153]$, as well as the 2DEG's unique characters in metalinsulator transition [56], superconductivity [58, 154-156], magnetism [57, 157-160] and optical responses [61,161-163] have evoked widespread interest. A mechanism involving the charge transfer between the surface and the interface ("polar catastrophe") was proposed to explain the formation of 2DEG [63,64]. Possible carrier depletion by oxygen dangling bonds at the surface was also suggested [67]. Different capping layers were explored to allow further tuning of the interface properties [68-70]. Effects of surface adsorbates formed in air are found to have significant role in interface conductivity. Among the various airborne molecules, the dissociation and migration of water molecules on oxide surfaces bear particular technological importance $[78,164-166]$. In $\mathrm{LaAlO}_{3} / \mathrm{SrTiO}_{3}$ heterostructures, effects of liquid water on the interface electrical properties have been experimentally observed [71,72]. In addition, reversible interface metal-insulator transitions controlled by conducting atomic force microscope (c-AFM) were previously reported [59-61,73,167]. Experiments performed in controlled environments later linked the effects directly to the surface water [74] [168]. 
Despite of these interesting experimental findings, clear understanding of the underlying mechanism is still lacking. Several critical questions remains open: How are water molecules adsorbed on $\mathrm{LaAlO}_{3}$ surfaces and is the adsorption energetically stable? How do the surface dangling bonds and water adsorptions in air affect the metal-insulator transitions observed in heterostructures with different $\mathrm{LaAlO}_{3}$ layer thicknesses? What are the dynamic processes of water adsorption and desorption? How do the adsorbed water respond to the external field or environment changes? In this work, we seek to shed light onto these open questions by systematic theoretical and experimental investigations. A partially dissociated water layer is found to strongly bond to the polar $\mathrm{LaAlO}_{3}$ surface. The densities and distributions of adsorbed protons and hydroxides strongly affect the structural and electrical properties of the $\mathrm{LaAlO}_{3} / \mathrm{SrTiO}_{3}$ heterostructures. Intrinsic interface carrier doping depending on the $\mathrm{LaAlO}_{3}$ thickness and extrinsic contributions to the interface metallicity from dissociated water are both observed. The weakly adsorbed molecular water layers were also found to play play important role in the associated hydrogen bond network which enables active surface proton transfer at room temperature. The tunable thermally activated proton hopping processes provide an effective pathway for engineering the interface properties from the surface. The multifaceted role of water revealed by this work is undoubtedly critical in understanding the unique characters at $\mathrm{LaAlO}_{3} / \mathrm{SrTiO}_{3}$ interfaces observed so far. Our results are also expected to provide valuable guidelines for the future development of oxides based surface engineering methodologies and device applications. 


\subsection{Experimental Methods}

\subsubsection{DFT calculations}

Density Functional Theory (DFT) calculations were performed as implemented in the Vienna Ab-initio Simulation Package (VASP) $[169,170]$ by Aldo H. Romero (West Virginia University) and A. C. Garcia-Castro (Centro de Investigación y Estudios Avanzados del IPN). Projector Augmented Wave (PAW) [171] pseudo-potentials were used to represent the valence and core electrons. The electronic configurations taken into account in pseudo-potential as valence electrons were $\operatorname{Sr}\left(4 \mathrm{~s}^{2} 4 \mathrm{p}^{6}\right.$ $\left.5 \mathrm{~s}^{2}\right)$, Ti $\left(3 \mathrm{~s}^{2} 3 \mathrm{p}^{6} 4 \mathrm{~s}^{2} 3 \mathrm{~d}^{2}\right)$, La $\left(4 \mathrm{~s}^{2} 4 \mathrm{p}^{6} 5 \mathrm{~d}^{1} 6 \mathrm{~s}^{2}\right), \mathrm{Al}\left(3 \mathrm{~s}^{2} 3 \mathrm{p}^{1}\right)$ and $\mathrm{O}\left(2 \mathrm{~s}^{2} 2 \mathrm{p}^{4}\right)$. The exchange correlation was represented within the General Gradient Approximation (GGA) and Perdew-Burke-Ernzerhof for Solids (PBEsol) parametrization [172]. Due to the magnetic character of these systems, the spin polarization was considered in the calculation with the proper use of an exchange correlation functional (LSDA). The periodic solution of these crystalline structures was represented by Bloch states with a Monkhorst-Pack with a k-point mesh and an of energy cut-off, tested to give converged forces to less than $0.001 \mathrm{eV} / \AA$. To correctly describe the system, $5 \mathrm{uc}$ of $\mathrm{SrTiO}_{3}$ (STO) as a substrate was taken into account. Then, 3 uc and 5 uc of $\mathrm{LaAlO}_{3}(\mathrm{LAO})$ were used as thin films for the analysis. Additionally, in order to avoid periodic interactions between periodic slabs, we used a supercell with a vacuum space of $12 \AA$ along the z-axis. The dipole correction was applied along the out-of-plane z-axis to cancel dipole-dipole interactions and correct the errors caused by periodic boundary conditions [173]. In addition and in order to take into account the octahedral rotations and tilting, we considered a cell in the xy-plane with lattice parameter fixed to the PBEsol relaxed STO a $=3.8967 \AA$. 


\subsubsection{Heterostructure synthesis}

The $\mathrm{LaAlO}_{3}$ thin films were deposited on $\mathrm{TiO}_{2}$ terminated (001) $\mathrm{SrTiO}_{3}$ substrates via pulsed laser deposition (PLD) at $550^{\circ} \mathrm{C}$ under $10^{-3} \mathrm{mBar} \mathrm{O}_{2}$ pressure by Chang-Beom Eom, Sangwoo Ryu (University of Wisconsin, Madison). Ohmic contacts to the $\mathrm{LaAlO}_{3} / \mathrm{SrTiO}_{3}$ interface are obtained by sputtering electrodes as follow: the electrodes are patterned via optical lithography; $7 \mathrm{~nm}$ depth of material is milled away in the electrode areas using $\mathrm{Ar}^{+}$ion etching; etched areas are backfilled with $2 \mathrm{~nm}$ Ti and $6 \mathrm{~nm} \mathrm{Au}$.

\subsubsection{X-ray photoemission spectroscopy}

Physical Electronics PHI 5000 VersaProbe XPS is used to analyze the chemical compositions and chemical bonds at the sample surfaces. XPS spectra are collected at room temperature in base pressure below $1.6 \times 10^{-6}$ Torr using $\mathrm{Al} K \alpha$ source $(1486.6 \mathrm{eV})$.

\subsubsection{Oxygen plasma surface treatment}

March PX-250 Plasma Asher is used for the oxygen plasma surface treatment. The following conditions are used in the experiments: RF (radio frequency) of 13.56 $\mathrm{MHz}, \mathrm{RF}$ power of $50 \mathrm{~W}$, oxygen flow rate of $50 \mathrm{sccm}$ (standard cubic centimeter per minute), base pressure of 80 mTorr, processing pressure of $\sim 300$ mTorr, DC bias of $5 \mathrm{~V}$ and exposure time of 1 minute. 


\subsubsection{C-AFM lithography, low temperature transport and photoconductivity measurements}

Contact mode c-AFM lithography is performed in atmosphere at room temperature using Asylum Research MFP-3D AFM system with platinum coated Si probe. Detailed method can be found in Ref [59,73]. Quantum Design Physical Properties

Measurement System (PPMS) is used for variable temperature transport measurements in Van der Pauw configurations. 


\subsection{Results}

\subsubsection{Water dissociation at the polar surface of $\mathrm{LaAlO}_{3} / \mathrm{SrTiO}_{3}$}

\section{heterostructures}
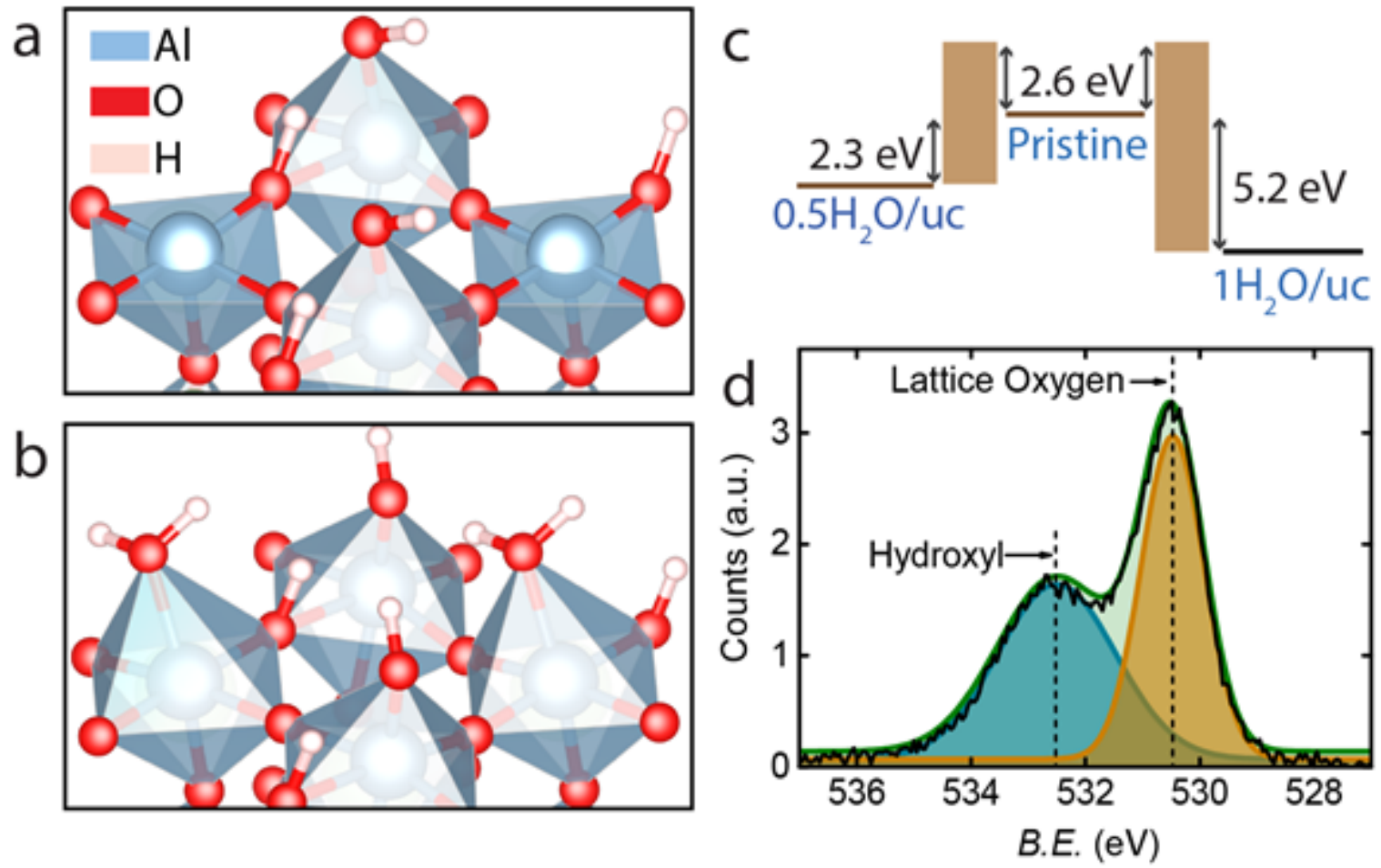

Figure 6.1: Water dissociation at $\mathrm{AlO}_{2}$ terminated $\mathrm{LaAlO}_{3}$ surface. (a) Simulated final state when an average of $0.5 \mathrm{H}_{2} \mathrm{O}$ molecules per $\mathrm{AlO}_{2}$ are released to the pristine $\mathrm{AlO}_{2}$ surface. The released water molecules dissociate completely and form Al-OH and $\mathrm{O}-\mathrm{H}$ bonds on the surface. (b) Simulated final state when an average of 1 $\mathrm{H}_{2} \mathrm{O}$ molecules per $\mathrm{AlO}_{2}$ are released to the pristine $\mathrm{AlO}_{2}$ surface. Only half of the released water molecules dissociate and the rest strongly bond to Al sites (Al$\mathrm{OH}_{2}$ ) in the molecular form. (c) Energy differences (per unit cell) between the initial pristine surface state and the final states illustrated in (a,b). Also included is the water ionization energy barrier of $2.6 \mathrm{eV}$. (d) Oxygen $1 s$ XPS spectra taken at $\mathrm{LaAlO}_{3} / \mathrm{SrTiO}_{3}$ heterostructure surface. Clear signature of surface hydroxyl group is observed, indicating the dissociative absorption of water.

Figure 6.1 (d) plots the Oxygen $1 s$ state X-ray photoemission spectroscopy (XPS) spectra acquired in a sample with 5 unit cell (uc) $\mathrm{LaAlO}_{3}$ grown on $\mathrm{SrTiO}_{3}$ substrate 
(5 uc LAO/STO). Spectra obtained from 3 uc and 10 uc LAO/STO samples are very similar. Two primary peaks can be observed. The narrower peak at $530.5 \mathrm{eV}$ is associated with oxygen atoms in the oxide lattice, while the wider peak of larger (by around $2 \mathrm{eV}$ ) bonding energy is the signature of hydroxyl (OH) group [174]. The large concentration of surface hydroxyl groups is a strong indication of the dissociative adsorption of water. No significant trace of molecular water (bonding energy larger than hydroxyl) is observed, which is probably due to the $10^{-6} \mathrm{mbar}$ vacuum level in the XPS chamber. However, in atmosphere with a humidity of 20\%$40 \%$, besides of the first chemisorbed water layer, additional molecular water layers will also be present [175].

To investigate the energetically preferred water dissociation configurations, density functional theory (DFT) calculations are performed for heterostructures with $3 \mathrm{uc}$ and $5 \mathrm{uc}$ LAO layers. In both cases, when an average of $0.5 \mathrm{H}_{2} \mathrm{O}$ molecule per $\mathrm{AlO}_{2}$ unit cell $\left(0.5 \mathrm{H}_{2} \mathrm{O} / \mathrm{uc}\right)$ are released to the pristine surface (Fig.6.1a), all the water molecules dissociate and form surface O-H/Al-OH bonds. For simplicity, we refer to this surface configuration as " $0.5 \mathrm{H}+0.5 \mathrm{OH}$ ". When the initial water density increases to $1 \mathrm{H}_{2} \mathrm{O}$ /uc, only half of the water molecules are dissociatively adsorbed (Fig.6.1b). The rest half are chemisorbed to the $\mathrm{Al}$ sites in molecular form $\left(\mathrm{Al}-\mathrm{OH}_{2}\right)$. We refer to this configuration as " $0.5 \mathrm{H}+0.5 \mathrm{OH}+0.5 \mathrm{H}_{2} \mathrm{O}$ ". The relative energy changes with or without dissociative water adsorptions as well as the water ionization energy barriers are illustrated in figure 6.1c. In both cases, the binding energies associated with dissociated water are around $4.8 \mathrm{eV}(\mathrm{O}-\mathrm{H})$ and $5 \mathrm{eV}(\mathrm{Al}-$ $\mathrm{OH})$. This high energy value indicate that, when there are less than one monolayer of water coverage, the dissociated $\mathrm{H}$ and $\mathrm{OH}$ are very strongly bonded to the surface. When more water molecules are released, dissociated water and molecular water form 
a complex network linked by hydrogen bonds.

\subsubsection{Effects of surface water adsorption on the interface properties}
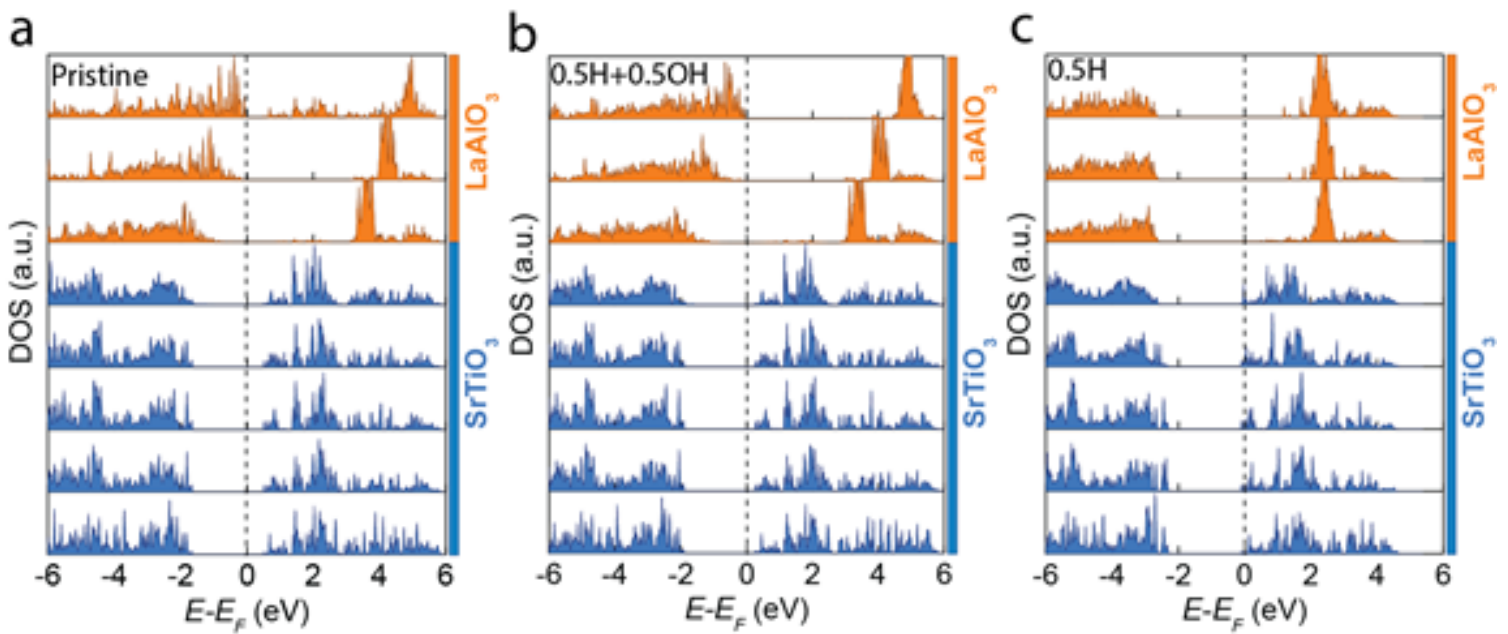

Figure 6.2: Layer resolved electronic density of states (DOS) in 3 uc LAO/STO heterostructure for surface absorptions illustrated in figure 6.2 (a) pristine $\mathrm{AlO}_{2}$ terminated surface with only physically absorbed water layer, (b) surface with $0.5 \mathrm{OH} / \mathrm{us}$ plus $0.5 \mathrm{H} / \mathrm{uc}$ absorbed, and (c) surface with only $0.5 \mathrm{H} / \mathrm{uc}$ absorbed. 

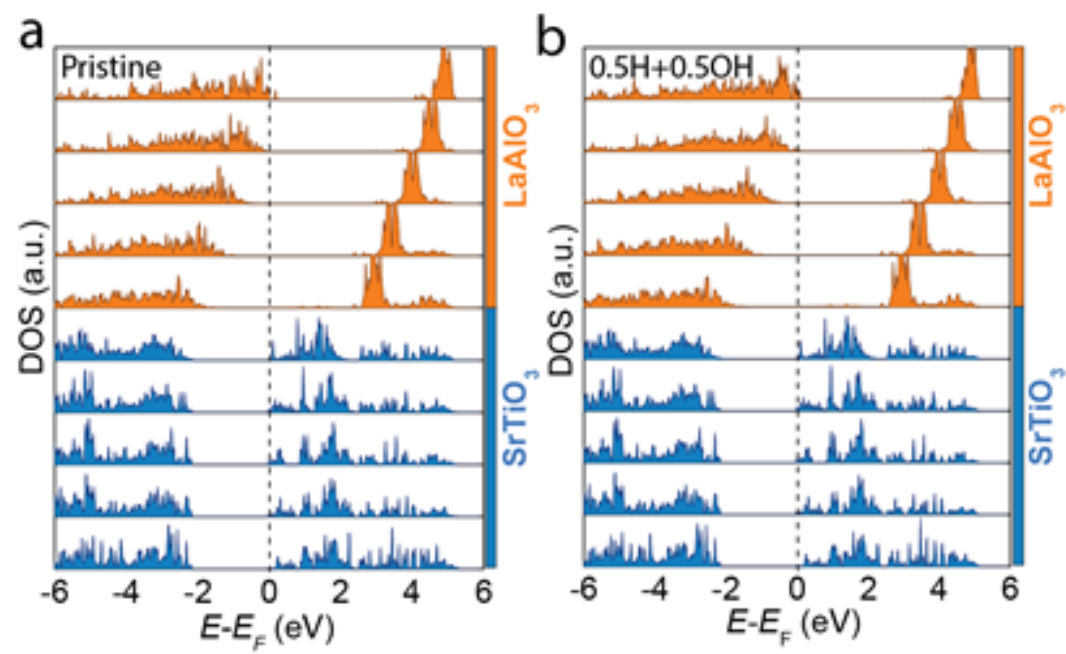

Figure 6.3: Layer resolved electronic density of states (DOS) in 3 uc LAO/STO heterostructure for surface absorptions illustrated in figure 6.2 (a) pristine $\mathrm{AlO}_{2}$ surface, (b) surface with $0.5 \mathrm{OH} /$ us plus $0.5 \mathrm{H} / \mathrm{uc}$ absorbed

Layer resolved density of states (DOS) calculated for 3 uc LAO/STO with a pristine surface and $0.5 \mathrm{H}+0.5 \mathrm{OH}$ surface are presented in figure $6.2 \mathrm{a}$, b. Heterostructure with $0.5 \mathrm{H}+0.5 \mathrm{OH}+0.5 \mathrm{H}_{2} \mathrm{O}$ surface exhibits results very similar to figure $6.2 \mathrm{~b}$. Two most obvious effects of the water dissociation are: the quenching of in-gap LAO surface states and the small bandgap reduction in STO layers close to the interface. In all three cases, Fermi level falls inside the bandgap in STO layers, indicating insulating interfaces. This is in consistence with the experimental reports that a critical LAO thickness of $d_{c}=4 \mathrm{uc}$ is required for the 2DEG formation [56]. Calculations performed for 5 uc LAO/STO show very different results. In pristine surface case, interface Fermi level resides at the bottom of the conduction band (Fig.6.3a). And water dissociations push the conduction band edge further below the Fermi level (Fig.6.3b). This effect is more obvious when we look at the total density of conduction band states below the Fermi level $(n)$. This parameter is related to the carrier 
density measured in experiments. $n$ in the STO interface layers are plotted in figure 6.4b,c. In 5 uc LAO/STO with pristine surface (labeled VI in figure 6.4a), $n$ peaks in the third STO layer (counting from the interface) (Fig.6.4c). Surfaces with water dissociations (labeled III and IV in figure 6.4a) further enhances $n$ (Fig.6.4c). In all three cases, $n$ of all STO layers remain zero in $3 \mathrm{uc} \mathrm{LAO/STO} \mathrm{(Fig.6.4b).}$
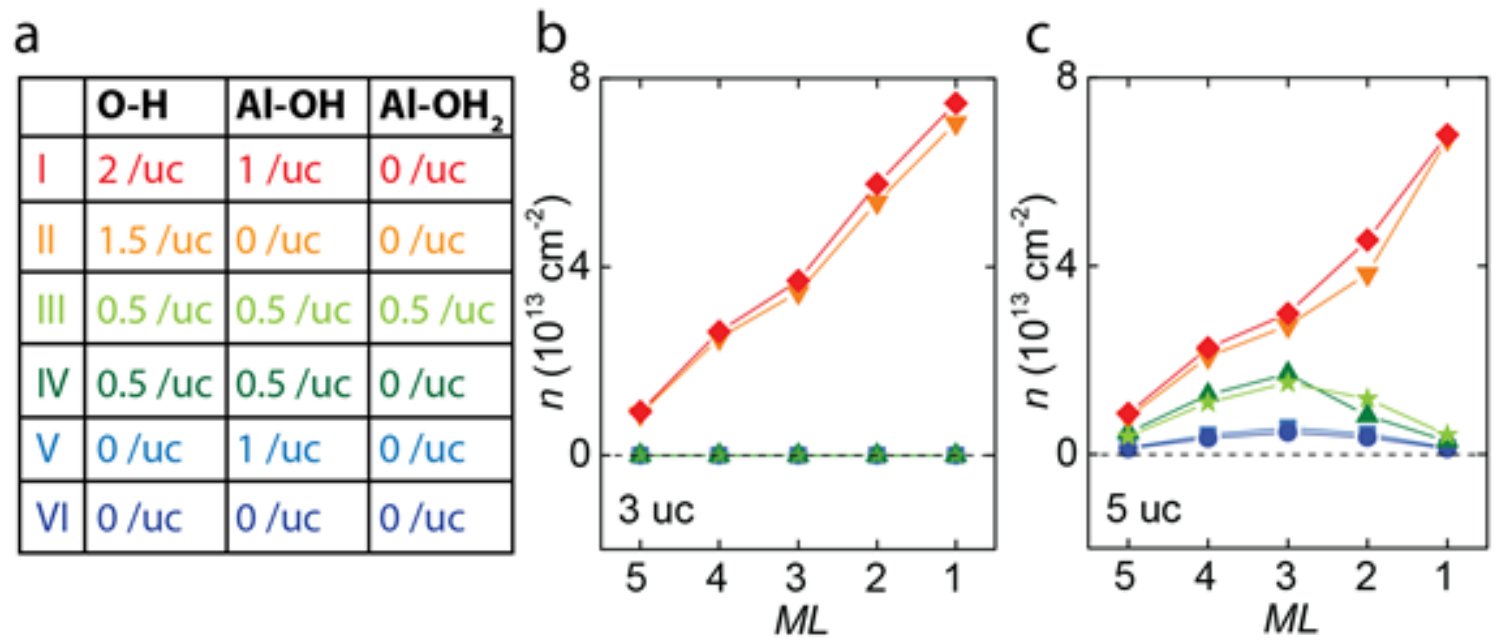

Figure 6.4: Total densities of conduction band states below Fermi level $(n)$ in $\mathrm{SrTiO}_{3}$ monolayers (ML) calculated for 3 uc $\mathrm{LaAlO}_{3} / \mathrm{SrTiO}_{3}$ (b) and $5 \mathrm{uc} \mathrm{LaAlO}_{3} / \mathrm{SrTiO}_{3}$ (c). The monolayer closest to the interface is labeled with number "1".Different colors indicate six surface adsorption states as listed in (a).

Among the three types of surfaces discussed above, a common property is that the density of surface adsorbed $\mathrm{H}$ and $\mathrm{OH}$ are equal $\left(n_{H}^{S}=n_{O H}^{S}\right)$. Allowing $n_{H}^{S}$ and $n_{O H}^{S}$ to be different produces more dramatic DOS changes in both 3 uc and 5 uc LAO/STO. For example, when the densities are $n_{H}^{S}=0.5 /$ uc and $n_{O H}^{S}=0$ at a 3 uc LAO surface, the built-in potential across the polar LAO layers is balanced and the conduction band edges of interface STO layers are pushed below the Fermi level (Fig.6.2c). This result suggests a surface proton adsorption induced interface metalinsulator transition in $3 \mathrm{uc} \mathrm{LAO/STO.} \mathrm{Calculations} \mathrm{for} \mathrm{more} \mathrm{surface} \mathrm{adsorption}$ configurations that are energetically stable are shown in Figure 6.4. When $n_{H}^{S}>n_{O H}^{S}$ 
(case I and II), $n$ increases enormously and the maximum of $n$ shifts to the STO layer closest to the interface. When $n_{O H}^{S}>n_{H}^{S}$ (case V), effects of protons are somewhat canceled and the distributions of $n$ are restored to approximately the same as in the heterostructures with pristine surface. We also note that, as $n_{H}^{S}$ increases, lattice distortion (tiltation of oxygen octahedra) becomes more and more significant (Fig.6.5).
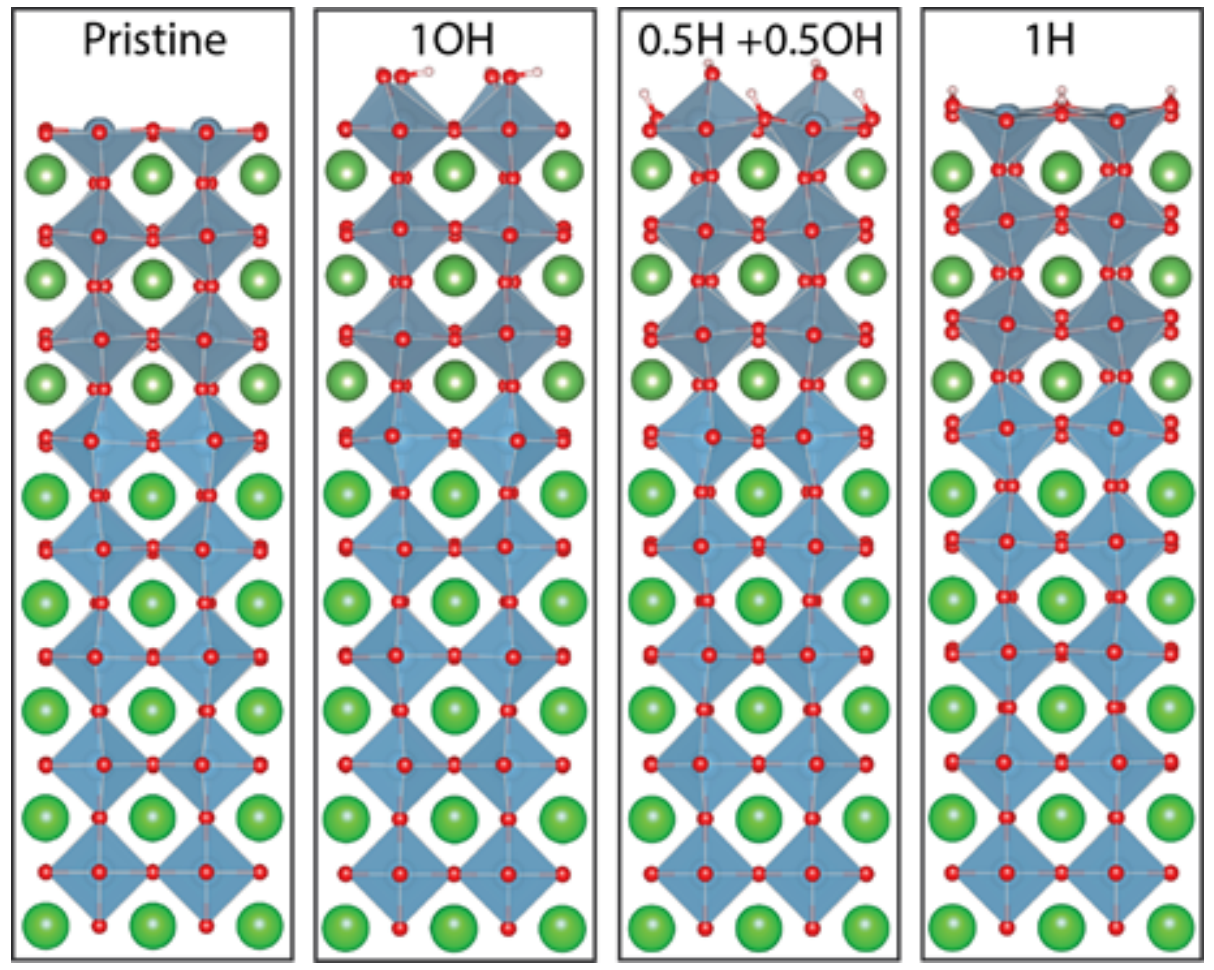

Figure 6.5: Simulated lattice distortions in $3 \mathrm{uc} \mathrm{LaAlO}_{3} / \mathrm{SrTiO}_{3}$ heterostructure with different surface $\mathrm{H}$ and $\mathrm{OH}$ densities. Tiltation of oxygen octahedra becomes more significant as the surface proton density increases.

Generally speaking, Larger $n_{H}^{S}$ tends to produce larger $n$ in STO interface layers. However, the distribution of protons at the LAO surface also plays an important role in determining the interface properties. Four different surface configurations with $n_{O H}^{S}=0$ and $n_{H}^{S}=0.5 / \mathrm{uc}, 1 / \mathrm{uc}$ and $1.5 / \mathrm{uc}$ are illustrated in figure $6.6 \mathrm{a}$, and their corresponding $n$ distributions in STO layers are plotted in figure 6.6c. Having the 
same surface proton density but different proton distributions, configurations i and ii lead to very different interface $n$ values. In the meanwhile, noticeable differences in lattice distortion and surface $\mathrm{O}-\mathrm{H}$ bond orientations are also present in the two cases (Fig.6.6b).
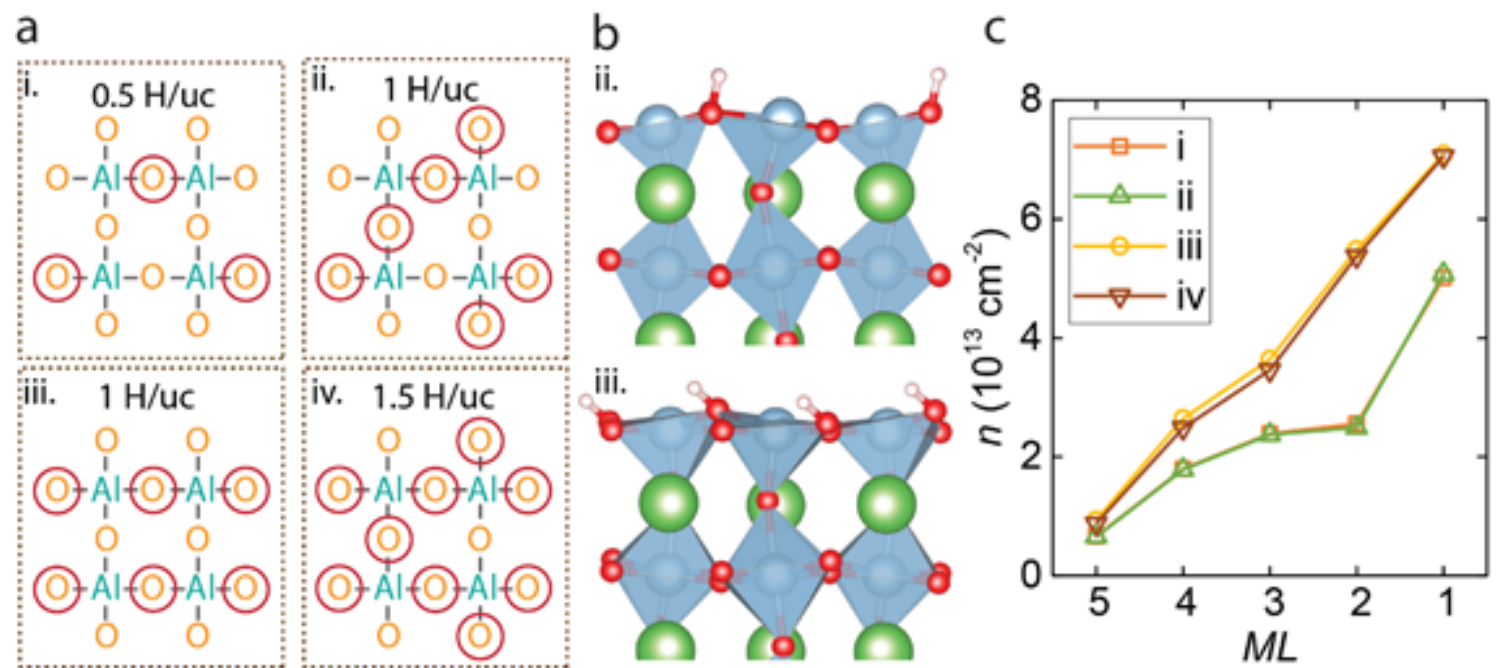

Figure 6.6: Total densities of conduction band states below Fermi level (c) and lattice distortions (b) resulted from different surface proton distributions as illustrated in (a). Oxygen sites with proton adsorbed are marked with red circles. 


\subsubsection{Modifying interface properties by oxygen plasma sur- face treatment}
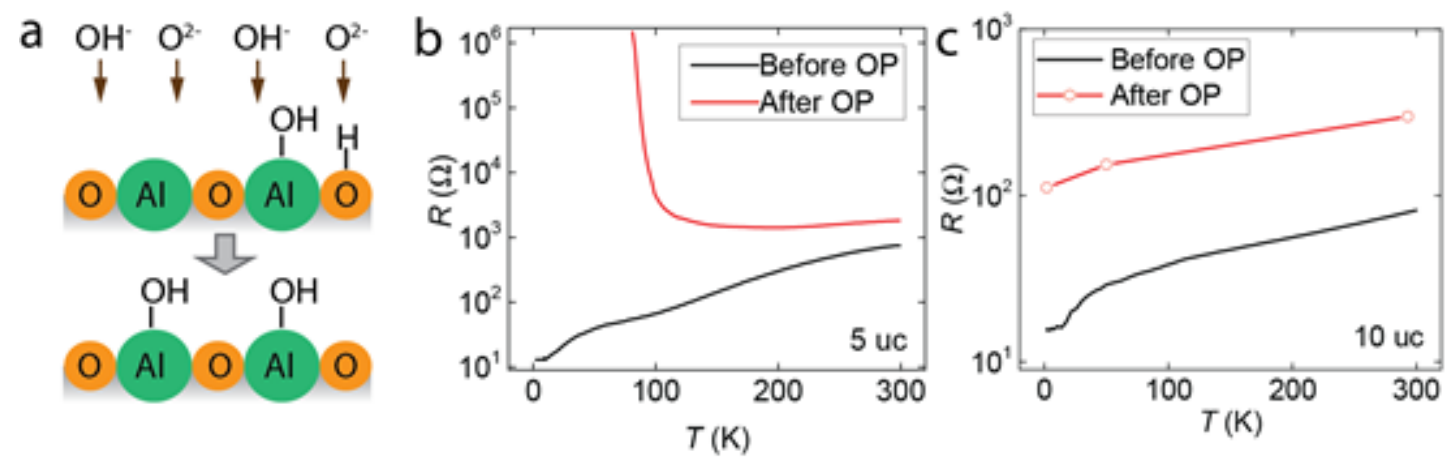

Figure 6.7: Suppression of the interface conductance induced by oxygen plasma (OP). (a) Illustration of the $\mathrm{Al}$ site terminations and oxygen site deprotonations occurring when LAO surface is exposed to the anions in oxygen plasma $\left(\mathrm{O}_{3}^{-}, \mathrm{OH}^{-}\right.$, etc.). (b,c) Temperature dependence of interface resistance measured in $5 \mathrm{uc}$ (b) and $10 \mathrm{uc}$ (c) $\mathrm{LaAlO}_{3} / \mathrm{SrTiO}_{3}$ heterostructures before and after the oxygen plasma treatment.

DFT calculations show that it is possible to engineer the LAO/STO interface properties by controlling the values of $n_{H}^{S}$ and $n_{O H}^{S}$. Experimentally, two methods were found effective for this task. The first method is exposing the sample surfaces to oxygen plasma $(\mathrm{OP})$, where a large density of negative ions or radicals $\left(\mathrm{O}_{3}^{-}\right.$, $\mathrm{OH}^{-}$, etc.) are driven to the sample surface. The very low energy ions in $\mathrm{OP}$ is expected to alter only the surface bonds and leave the bulk material unaffected. This is confirmed by the subsequent AFM imaging and XPS measurements, which show no change in surface topography or metallic elements compositions. Two main reaction processes are expected between the OP anions and the LAO surfaces: one is the termination of the surface $\mathrm{Al}$ dangling bonds $(\mathrm{Al}+\mathrm{OH} \rightarrow \mathrm{Al}-\mathrm{OH})$ and the second is the surface deprotonation $\left(\mathrm{O}_{(L A O)} \mathrm{H}+\mathrm{OH} \rightarrow \mathrm{O}+\mathrm{H}_{2} \mathrm{O}\right)$ (Fig. 6.7a). The net effects lead to larger $n_{O H}^{S}$ and smaller $n_{H}^{S}$, favoring the suppression of interface conductance. The transport measurements before and after the OP treatments are in 
very good agreement with the theory (Fig.6.7b,c). Temperature dependent resistance of the 5 uc LAO/STO sample exhibits a metallic behavior before the OP treatment, but afterwards transits to insulating at low temperatures (Fig.6.7b). An identically treated 10 uc sample exhibits only a large increase in resistance but remains metallic. Interface resistances in both samples remain large even after days' exposure to the air, indicating that the OP induced surface changes are long-lasting. Another implication is that water adsorptions also contribute to the 4 uc critical LAO thickness $\left(d_{c}\right)$ required for $2 \mathrm{DEG}$ formation. Without the surface water layer, $d_{c}$ may become larger.

\subsubsection{Effects of c-AFM lithography}
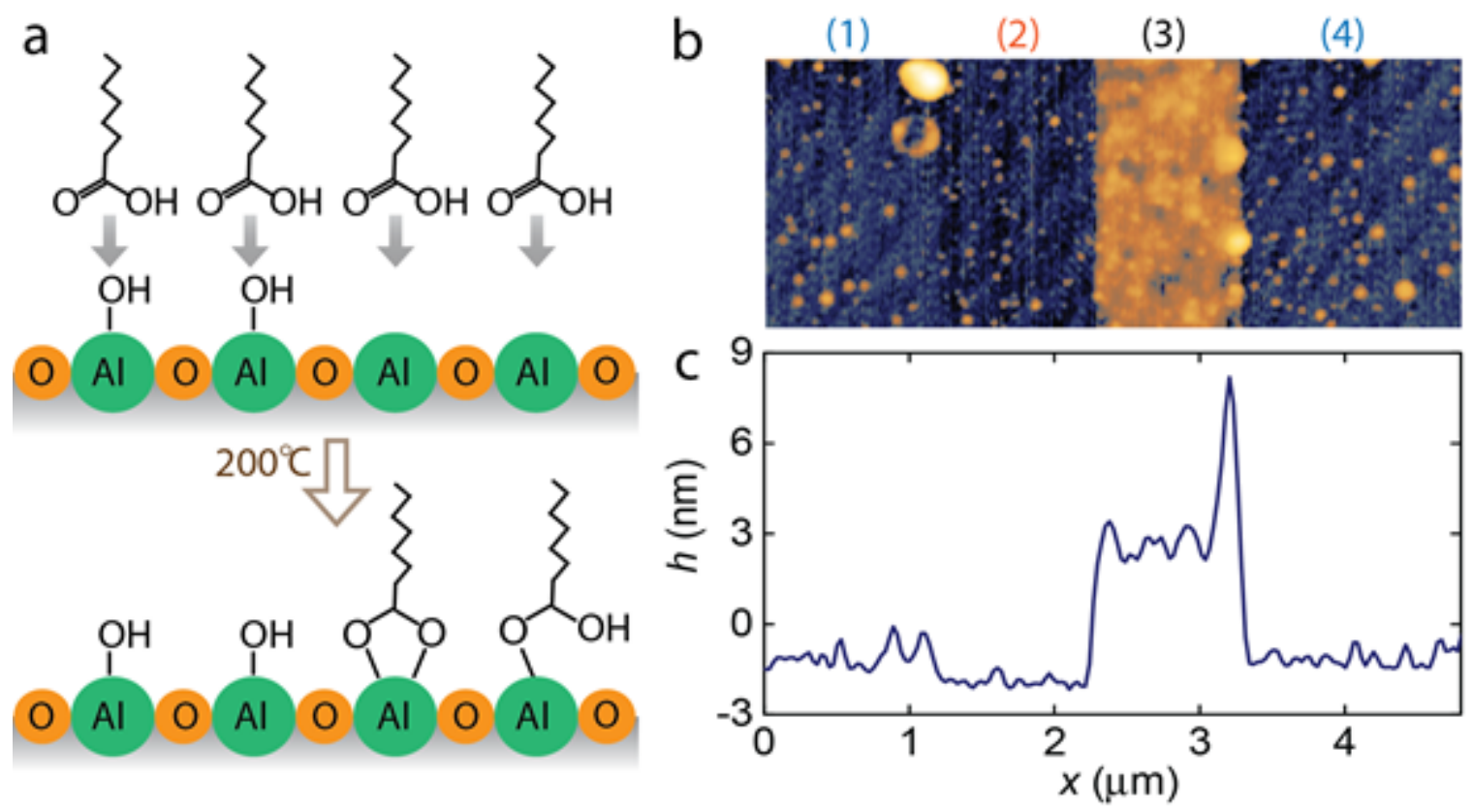

Figure 6.8: B(a) Illustration showing the bonding of oleic acid molecules onto unterminated surface $\mathrm{Al}$ sites. (b) AFM topography image taken after three consecutive steps: c-AFM writing $\rightarrow$ oleic acid (OA) introduction $\rightarrow 200^{\circ} \mathrm{C}$ baking. Region (1) and (4) were scanned by $10 \mathrm{~V}$ probe bias. Region (2) was scanned by $-10 \mathrm{~V}$. Region (3) was not touched during the c-AFM step. (c) Averaged line scan data extracted from (b). 
Besides oxygen plasma, biased c-AFM lithography has also been proven useful in reversibly control the interface properties in $3 \mathrm{uc}$ LAO/STO samples [59,73]. A "water cycle" mechanism was previously proposed to explain such effects [59, 74], where positively biased probe is expected to remove the surface adsorbed $\mathrm{OH}$ to promote the interface metallicity and negatively biased probe is expected to remove the surface proton and suppress the interface conductivity. Here we put this proposed theory to test by investigating the changes of surface Al-OH bonds induced by cAFM lithography. In the experiments, molecules that can also chemibond to the $\mathrm{Al}$ sites were introduced to the c-AFM scanned surfaces. Depending on the density of vacant surface $\mathrm{Al}$ sites after the c-AFM scans, adsorption of the artificially introduced molecules will vary. And the results were studied by the subsequent topography imaging. Oleic acid (OA), a large organic molecule containing a polar carboxyl group $(-\mathrm{C}(=\mathrm{O}) \mathrm{OH})$ and a long hydrocarbon chain, was chosen for this purpose. OA molecules can bond to the $\mathrm{Al}$ sites through oxygen atoms in the carboxyl group [176] (Fig.6.8a), and the large molecule size (monolayer thickness around $2 \mathrm{~nm}$ ) can produce significant topographical features that are easy to measure by AFM. Starting from a sample with clean as-grown surface, c-AFM lithography was first performed in several regions with different biases. Then, a droplet of OA liquid was introduced to the surface. The sample was later baked dry at $200^{\circ} \mathrm{C}$ in air to remove the loosely condensated OA layers. One typical AFM tapping mode image obtained at baked sample surface is shown in Figure 6.8b. During the c-AFM lithography step, probe biases used were $10 \mathrm{~V}$ for region 1,4 and $-10 \mathrm{~V}$ for region 2. Region 3 was not scanned during the c-AFM lithography step. Clear height differences between these regions were observed in the final topography imaging (Fig.6.7b). OA was mainly adsorbed in areas that have never been scanned by biased c-AFM probe (region 3), 
where two monolayers (ML) of OA form. In the previously scanned regions (region $1,2,4)$, regardless of the bias polarity used, only scattered OA islands were found and the terrace structure of clean LAO surface can be clearly seen. The greatly suppressed OA adsorption by biased c-AFM scan suggests that both positive and negative probe biases lead to surfaces with more terminated $\mathrm{Al}$ sites (larger $n_{O H}^{S}$ ). Such observation is obviously not consistent with the previous proposed mechanism where $\mathrm{OH}$ is removed from $\mathrm{Al}$ sites by positive probe biases. More likely, it is the increase (positive biases) or decrease (negative biases) of $n_{H}^{S}$ that is predominantly responsible for the interface metallicity control.

Unlike the conductance suppression produced by oxygen plasma, interface metallicity created by positive probe biases is only stable in vacuum or at low temperatures $[59,71,72,160]$. At room temperature in air, the large interface conductance typically decays within one hour after the c-AFM scan (Fig. 6.10d,e, dash line). To shed more light onto the decay process, we studied the changes of interface conductance in solvent environments with different proton densities and solvabilities. Three types of liquid chemicals were used: I. molecules with labile protons and can also establish hydrogen bonds with protons (DI water, methanol, isopropanol, acetic acid, propionic acid); II. Molecules without labile proton but still can establish hydrogen bonds to protons (acetone, ethyl acetate, acetonitrile); III. Hydrocarbon molecules (heptane, pentane, toluene) which display neither labile protons nor hydrogen bonding. In this experiment, a conducting channel connecting two interface electrodes was first created by $10 \mathrm{~V}$ biased probe at the originally insulating interface of a 3 uc LAO/STO sample. As soon as the c-AFM scan finished (marked by the sudden increase of conductance between electrodes as can be seen in figure 6.10e), drops of selected liquid chemicals were introduced to cover the whole LAO surface. Two 
distinct types of behaviors were observed in the subsequent conductance measurements (Fig. 6.10d,e). Regardless of the labile proton concentration, type I and II liquids both diminished the interface conductance within seconds (Fig. 6.10d). The induced changes were non-reversible even after the surfaces were baked dry. In contrast, when the LAO surfaces were covered by type III liquids, the lifetime of the interface conductance was significantly prolonged (Fig. 6.10e). In this case, the monitored conductance decreased by only a small fraction within the $12 \mathrm{hr}$ measuring window. These observations indicate that, hydrogen bonding (i.e. proton solvability) plays an important role in the evolvement of LAO surfaces adsorption states.

\subsection{Discussion}

\subsubsection{Effects of charge transfer, electron correlations, and lattice distortions}

Different theoretical calculations have demonstrated that the electronic reconstruction induced by the polar catastrophe phenomena is the main responsible for the insulator-to-metal transition (IMT) in LAO/STO and the appearance of a 2DEG at the polar-nonpolar perovskite oxide interfaces (see for example Ref [177]). Therefore, modifications of the surface formal charges at the $\mathrm{AlO}_{2}$ top layer is expected to change the interfacial properties. Then, a clear understanding of this phenomenon is crucial in order to explain the macroscopic electronic transport properties discussed in this work. In the $3 \mathrm{uc}$ pristine case, the electric field of $54 \mathrm{mV} / \AA$ (computed from the layer resolved DOS) is clearly not enough to overcome the existing electron gap in the STO/LAO system, leading to the obtained insulated behavior $\left(E_{g}\right.$ $=0.5 \mathrm{eV})$. The water dissociation and the later removal of the $\mathrm{OH}^{-}$groups with 
the AFM tip leaves only absorbed hydrogen atoms on the surface which, according to our results, act as electron-donors. These additional electrons, under the effect of the electric field existing in the LAO film, are transferred to the interface, which then generate a 2DEG with $d_{x y}$ character from the Ti orbitals. This electron transfer explains the induced IMT in the 3 uc LAO, as depicted in Fig. 6.4b. Consequently, the absorbed hydrogen act as electron-donor and provide additional charge, which is enough to compensate the surface charge responsible for the polar discontinuity. The later clearly alters the out-of-plane electrostatic potential. For example, when the surface is fully hydrogen saturated, the field takes a value of $-86 \mathrm{mV} / \AA$ that is in opposite direction to the potential in the $\mathrm{AlO}_{2}^{-}$pristine surface. This field crosses through $0 \mathrm{mV} / \AA$ for $0.5 \mathrm{H}$ (see Fig.2), which demonstrates the direct effect of the electron-donors in the electrostatic response, as was expected. On the other hand, if we consider 5 uc of $\mathrm{LaAlO}_{3}$ on $\mathrm{SrTiO}_{3}$ (001), the electric field is now close to 110 $\mathrm{mV} / \AA$ across the entire LAO film. This value is sufficient to overcome the present electronic band gap of the STO material and the system becomes metallic, as it is widely known. Additionally, a 2DEG and a 2-dimensional hole gas (2DHG) are created at the interface and surface respectively. Now, with the same process of water absorption and later $\mathrm{OH}^{-}$removal, as in the previous 3 uc case, the hydrogen electron-donor alters the amount of electrons in the system. These additional electrons are transferred from the surface to the interface (similarly to the case of 3 uc of $\mathrm{LaAlO}_{3}$ ) and the amount of carriers density increase drastically as observed in the Fig. 6.4c, as it is also supported from experimental results. Our theoretical analyses also reveal the appearance of magnetic moments at the interface for the 5 uc case, as expected from the unpaired $3 d-\mathrm{Ti}$ orbitals occupation as it has been already reported [178]. In the 3 uc case, magnetic moments are only observed at the 
$3 d$-Ti when the interface becomes metallic due to the bonded hydrogen. When only OH-groups are in the LAO surface, the magnetism is only present at the top layer. This is due to the electron holes at the localized oxygen $p$-orbitals as it has been shown in some $d^{0}$ semiconductor [179].

Now, if we consider the possibility of reversing the voltage on the AFM tip, $\mathrm{H}^{+}$ is removed and $\mathrm{OH}^{-}$are kept on the surface, such that there is a reduction of the electric field in the LAO film. This field goes from $110 \mathrm{mV} / \AA$ to $87 \mathrm{mV} / \AA$ for the pristine structure to the $\mathrm{OH}$ saturated surface respectively. However, according to our results, this field reduction is not having a major effect in the carrier density as can be appreciated in Fig. 6.4. This can be explained by the fact that in the 5 uc, the charge is already confined at the interface and there is no charge transfer going back to the surface, which is already saturated with $\mathrm{OH}$. The later is traduced in an increase of the holes at the surface. In the 3 uc case, where the heterostructure is already an insulator, the electric field is also reduced to $54 \mathrm{mV} / \AA$ and the system kept a finite electronic band gap.

The fact that we are considering oxides in which, strong electron correlations takes place, makes important to investigate the effect in the results of the exchange correlation functional by enhancing the electron-electron repulsion in the so called $\mathrm{DFT}+\mathrm{U}[180]$. This method allows to increase the electron correlation lacking in the d-orbitals and then improving the electron band gap, in closer agreement with experimental predictions. This correction can also affect negatively the searched properties because it changes at the same time some other orbitals. Thus, we have considered initially the 3 uc case as a prototypical example. All results here were re-computed with a $\mathrm{U}=7.5$ and $5.8 \mathrm{eV}$ for $\mathrm{Ti}$ and La respectively and $\mathrm{J}=0 \mathrm{eV}$ [181]. We found that the general description holds but the valence carrier density increases 
by more than one order of magnitude, which is not in agreement with the reported experiments. Therefore, our results reported here only correspond to the case of $\mathrm{U}$ $=\mathrm{J}=0 \mathrm{eV}$. Either case, we consider that introducing the parameters $(\mathrm{J}, \mathrm{U})$ will gives us the option to enhance localization, where we expect to be able to drive the system from metallic to insulator. These parameters needs to be studied with more care and it will be the purpose of future work. 


\subsubsection{Roles of molecular water layers and the hydrogen bonds}

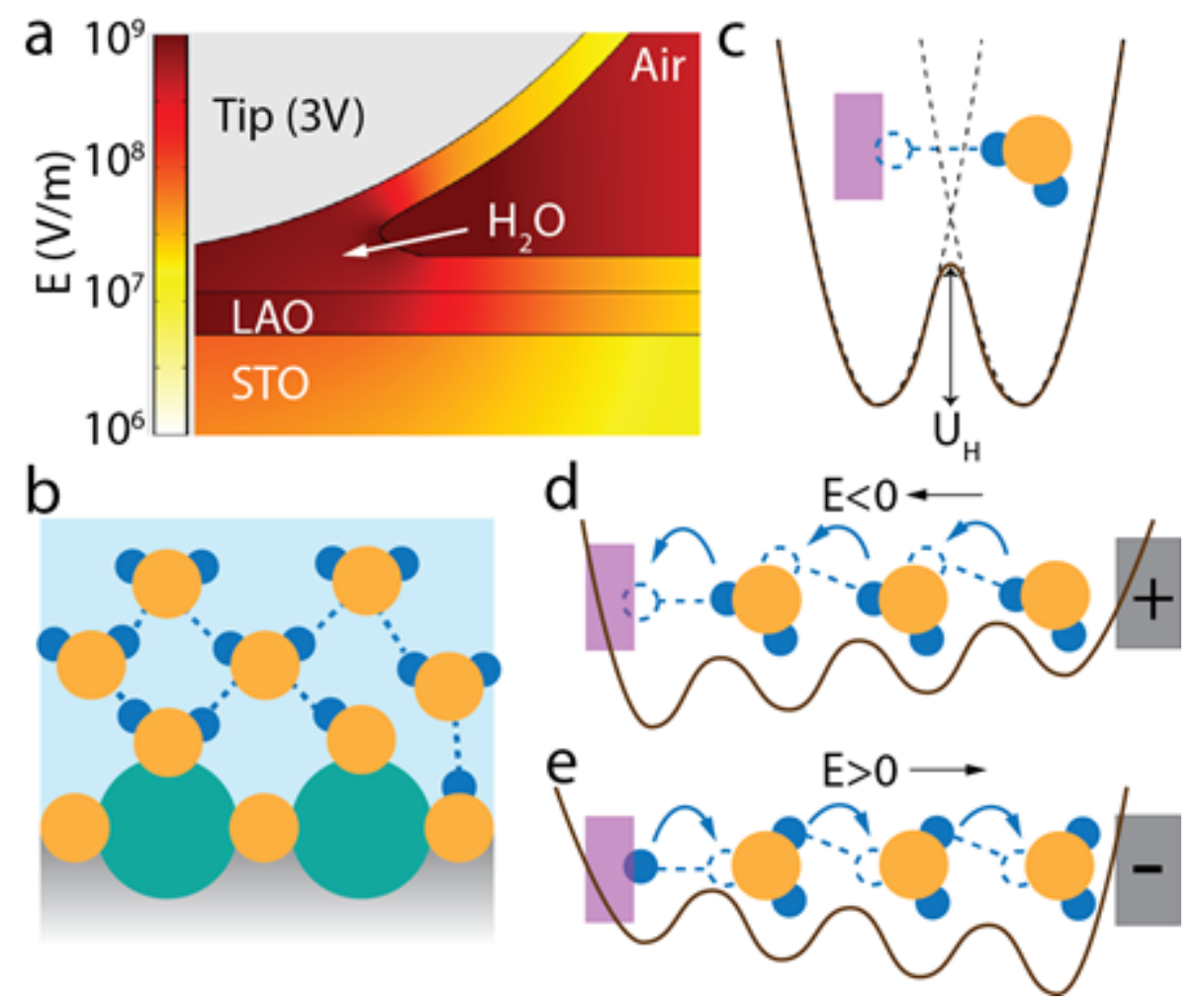

Figure 6.9: Biased AFM probe induced proton hopping. (a) Finite element simulation of the electric field generated when $3 \mathrm{~V}$ biased AFM probe (radius of curvature of $20 \mathrm{~nm}$ ) is in contact with the sample surface. $1 \mathrm{~nm}$ surface water condensation layer is assumed. (b) Illustration of the hydrogen bonding network formed at LAO surface covered with multiple water layers. (c) Illustration of the potential well crossing and resultant weakening of proton transfer barrier produced by the hydrogen bond between LAO surface and water molecules. (d) Positive tip biases accelerates the proton hopping toward the surface and while (e) negative biases result in the opposite trend.

Removal of an isolated surface proton involves overcoming a potential barrier of $4.7 \mathrm{eV}$. With a bond length of $1 \AA$, this requires a field of $E^{*} \approx 5 \times 10^{10} \mathrm{~V} / \mathrm{m}$. Probe field generated by typical biases used in c-AFM lithography was evaluated by finite element simulations (Fig. 6.9a). The result is more than one order of magnitude smaller than $E^{*}$. Similar problem exists when it comes to adding protons to LAO 
surfaces. Assuming water molecules are the main source of protons, retrieving a proton from an isolated water molecule also requires a similarly large energy of 5.1 $\mathrm{eV}$. Therefore, proton ionization pathways with much lower energy barriers need to be considered in order to explain the c-AFM manipulation of the surface proton density. The importance of hydrogen bonds revealed by the solvent tests provides an interesting clue. So far, all the discussions emphasize on the very first layer of dissociated water. Also important are the additional molecular water layers and the associated hydrogen bond networks [164,182], (Fig.6.9b). Through a hydrogen bond, the electropositive proton in one molecule and the electronegative atom in a second molecule are attracted to each other. The resultant crossing of proton binding potentials effectively lower the proton ionization energy barrier (Fig.6.9c). For example, the $5.1 \mathrm{eV}$ proton dissociation energy in isolated water molecules drops to $0.75 \mathrm{eV}$ in water networks $[183,184]$. Similar barrier reduction via hydrogen bond is also expected in the proton transfer between the LAO surface and the surface water molecules, which makes the room temperature manipulation of surface proton possible. The hopping exchange of protons between the LAO surface and the water layers can be described by:

$$
d n_{H}^{S} / d t=-\gamma_{H}^{S}(E) n_{H}^{S}+\gamma_{H}^{H_{2} O}(-E) n_{H_{2} O}+\gamma_{H}^{H_{3} O}(-E) n_{H_{3} O}+\gamma_{H}^{O H}(-E) n_{O H}
$$

$n_{H}^{S}$ is the density of protons adsorbed by LAO surface, $n_{H_{2} O}$ is the surface density of water molecules, $n_{H_{3} \mathrm{O}}$ and $n_{\mathrm{OH}}$ are the surface density of ions $\left(\mathrm{H}_{3} \mathrm{O}^{+}, \mathrm{OH}^{-}\right)$created during the proton hopping process. $\gamma_{H}^{S}(\mathrm{E}), \gamma_{H}^{H_{2} O}(E), \gamma_{H}^{O H}(E)$ and are the rates of proton dissociation from the LAO surface, water molecules and ions. These rates 
can be calculated by a thermally activated model:

$$
\gamma(E)=\operatorname{Aexp}\left[-\frac{U_{H}-E \Delta P}{k T}\right]
$$

, where $\Delta P$ is the change of dipole moment before and after proton transfer $[183,184]$. For example, using literature reported values of $U_{H}^{\mathrm{H}_{2} \mathrm{O}}, A^{\mathrm{H}_{2} \mathrm{O}}$ and $\Delta P^{\mathrm{H}_{2} \mathrm{O}}, \gamma_{\mathrm{H}}^{\mathrm{H}_{2} \mathrm{O}}$ is around $100 \mathrm{~s}^{-1}$ at room temperature under zero electric field [183,184]. Using the probe field calculated by finite element method (Fig.6.9a), $\gamma_{H_{2} O}$ dramatically increases to $10^{2} \mathrm{~s}^{-1}$ at $3 \mathrm{~V}$ probe bias, and $105 \mathrm{~s}^{-1}$ at $10 \mathrm{~V}$. Protons at LAO surface have slightly smaller binding energies comparing to protons in water molecules, and the bond lengths in both cases are similar. We expect $\gamma_{H}^{S}$ to be similar with $\gamma_{\mathrm{H}}^{\mathrm{H}_{2} \mathrm{O}}$ but possibly smaller. The general relation between all four rates is: $\gamma_{\mathrm{H}}^{\mathrm{H}_{3} \mathrm{O}}>>$ $\gamma_{H}^{\mathrm{H}_{2} \mathrm{O}}, \gamma_{\mathrm{S}}^{\mathrm{S}}>>\gamma_{H}^{\mathrm{OH}}$.

\subsubsection{Surface protonation/deprotonation driven by probe bi- ases}

Under the large electric field generated by the biased probe, $\gamma_{H}^{S}(E), \gamma_{H}^{H_{2} O}(E)$, $\gamma_{H}^{H_{3} O}(E)$ and $\gamma_{H}^{O H}(E)$ at the surface-water interface get either enhanced or suppressed depending on the field orientation. Another effect of the probe field is that the ions generated during surface-water proton transfer can be quickly pulled away from the surface. Also due to the multiple water layers formed under regular lab humidity levels, water molecules are abundant. Therefore, within a short time period right after the application of probe bias, $n_{\mathrm{H}_{3} \mathrm{O}}$ and $n_{\mathrm{OH}}$ can be considered negligible, and 
$n_{\mathrm{H}_{2} \mathrm{O}}$ can be treated as a constant. The solution to Eq. (6.1) is then:

$$
n_{H}^{S}(t)=\left[\left.n_{H}^{S}\right|_{t=0}-\frac{\gamma_{H}^{H 2 O}(-E)}{\gamma_{H}^{S}(E)} n_{H_{2} O}\right] e^{-\gamma_{H}^{S}(E) t}+\frac{\gamma_{H}^{H_{2} O}(-E)}{\gamma_{H}^{S}(E)} n_{H_{2} O}
$$

The surface proton density will be driven toward the equilibrium value:

$$
\left.n_{H}^{S}\right|_{e q}(E)=\frac{\gamma_{H}^{H_{2} O}(-E)}{\gamma_{H}^{S}(E)} n_{H_{2} O}=\left.\exp \left[\frac{-2 E \Delta P}{k T}\right] n_{H}^{S}\right|_{e q}
$$

with a characteristic time of $\tau=1 / \gamma_{H}^{S}$. At positive biases $(E<0), r=\exp \left[-\frac{2 E \Delta P}{k T}\right]$ is larger than one and the protonation of the surface leads to the local interface 2DEG formation (Fig.6.9d). At negative probe biases $(E>0), r$ is less than one, and the deprotonation of the surface leads to an insulating interface (Fig.6.9e). $\tau$ can be very short at positive probe biases. As discussed above, $\gamma_{S}^{H} \sim \gamma_{S}^{H_{2} O}$ can be as large as $10^{5}$ $\mathrm{s}^{-1}$ at a probe bias of $10 \mathrm{~V}$. This is consistent with the previous report that c-AFM writing can be achieved by voltage pulses as short as $10 \mu \mathrm{s}$ [73].

As the duration of the probe bias increases, a large density of ions will be created $\left(2 \mathrm{H}_{2} \mathrm{O} \rightarrow \mathrm{OH}^{-}+\mathrm{H}_{3} \mathrm{O}^{+}\right)$. After the biased probe moves away, these additional ions may diffuse to the LAO surface before they neutralize with each other. The contacts with these active ions are likely to produce more $\mathrm{Al}-\mathrm{OH}$ or $\mathrm{Al}-\mathrm{H}_{2} \mathrm{O}$ bonds at the LAO surface. As a result, surface $\mathrm{Al}$ sites in regions scanned by biased probe (regardless of the bias polarity) are always more completely terminated comparing to the unscanned regions. Such effects may explain the contrast of oleic acid adsorption observed in Figure 6.8. 


\subsubsection{Stability differences between surface bonded $\mathrm{H}$ and $\mathrm{OH}$}

The simple thermodynamic model can also well explain the lifetime variation of the c-AFM induced interface conductance in different environments. In type-I and II liquids, high density of hydrogen bonds enable very fast solvation/diffusion of protons [185-187]. For example, picosecond to nanosecond diffusion time constant is reported for proton diffusion in water-acetonitrile mixture [187]. Therefore, protons dissociated from sample surface will almost instantaneously diffuse into bulk liquid (Fig.6.10b), and ion densities $n_{H_{3} O}$ and $n_{O H}$ at the sample surface can be considered negligible. In this case, the evolvement of surface bond proton density $n_{H}^{S}(t)$ can be described by the zero field analogies of Eq.6.3 and 6.4. The surface proton density, starting from the high value after positively biased c-AFM scan, exponentially return to the zero field equilibrium $\left.n_{H}^{S}\right|_{e q}(0)=\frac{\gamma^{H 2 O}(0)}{\gamma_{H}^{S}(0)} n_{H_{2} O}$ with a characteristic time of $\tau=1 / \gamma_{H}^{S}(0)$. As a result, the interface is quickly restored to the insulating state prior to the c-AFM writing. In almost all of the type-I and II liquids tested, the interface conductance dropped in less than one second after the liquid introduction. This indicates that $\gamma_{S}^{H}(0) \sim \gamma_{S}^{H_{2} O}(0) \approx 10^{0} s^{-1}$, highly consistent with our previous estimation.

Type-III liquid molecules are mostly hydrocarbon and don't accept hydrogen bond. Solvation and diffusion of protons in them are very slow. The hydrocarbon layers also effectively prevent the particle exchange between the sample surface and the air (Fig.6.9 c). As a result, the sample surface and water layers, when covered by type-III liquids, can be considered as an isolated system. Once protons are transferred from LAO surface to water molecules, the generated hydronium ions are trapped in the system. As $n_{H_{3} \mathrm{O}}$ increases, the large value of $\gamma_{H}^{H_{3} O} n_{H_{3} O}$ will eventually 
slow down the surface deprotonation process. In this isolated system, applying element $(\mathrm{O}, \mathrm{H})$ conservation conditions and the relation of $\gamma_{H}^{\mathrm{H}_{3} \mathrm{O}}>>\gamma_{H}^{\mathrm{H}_{2} \mathrm{O}}, \gamma_{\mathrm{H}}^{\mathrm{S}}>>\gamma_{H}^{\mathrm{OH}}$ to Eq. (6.1), the surface proton density at equilibrium $\left(d n_{H}^{S} / d t=0\right)$ can be approximated as $\left.\left.n_{H}^{S}\right|_{e q} \approx n_{H}^{S}\right|_{t=0}$, consistent with the only slightly changed interface conductance measured in type III liquids. In the meanwhile, lateral proton hopping mediated by water molecules may still occur $[164,166]$, which can lead to the dilution of carrier concentration at the interface. Evaporation of hydrocarbon liquid is not considered in this idealized model either. Both of them can contribute to the slow interface conductance changes observed (Fig.6.10e). 
a

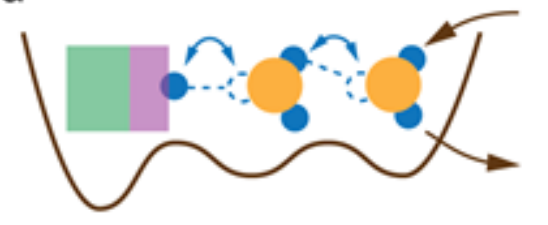

b)

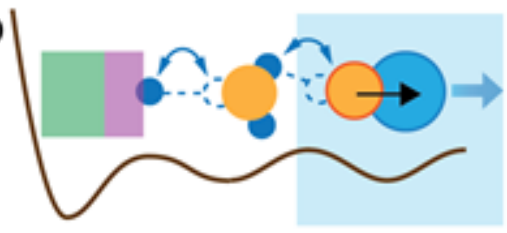

C

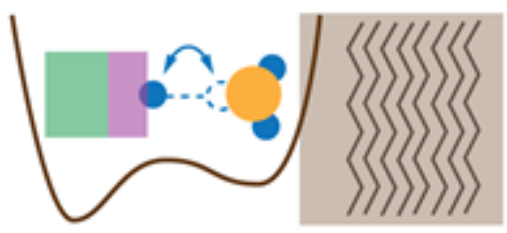

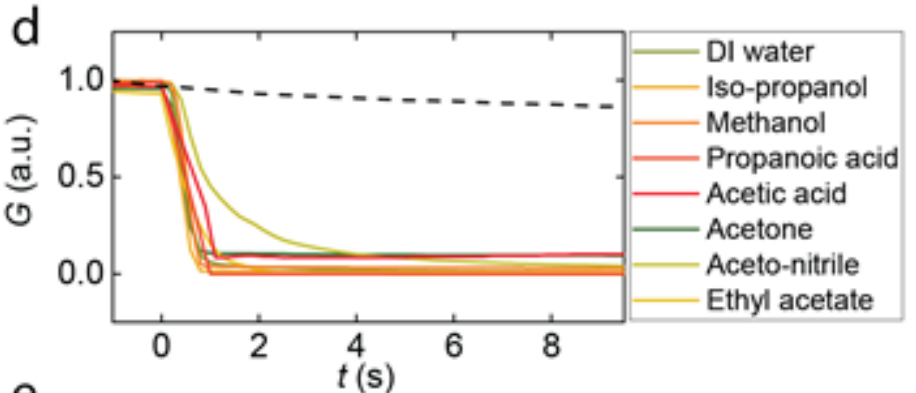

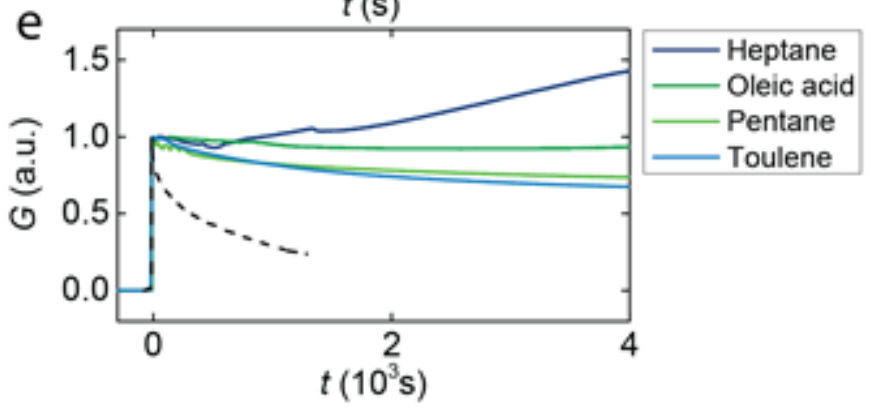

Figure 6.10: Retentions of the LAO surface $\mathrm{O}-\mathrm{H}$ bonds in different environments. (a) Exposed to air, the speed of deprotonation of $\mathrm{AlO}_{2}$ surface is controlled by particle exchange rate at the air-sample interface. A typical conductance decay curve followed by $10 \mathrm{~V}$ c-AFM writing in $3 \mathrm{uc} \mathrm{LAO/STO}$ is shown by black dash line in (d) and (e). (b) In solvents allowing hydrogen bonding, protons diffuse very fast through the hydrogen bond network. (d) Therefore, introducing these solvents to the LAO surface after c-AFM writing results in a fast surface deprotonation and a drop of interface conductance within seconds. (c) Hydrocarbon molecules don't allow hydrogen bond. They also block the particle exchange with air. (e) As a result, capping with hydrocarbon significantly prolong the lifetime of the conducting structures at LAO/STO interfaces.

The two cases discussed above represents the extreme situations where the dissipation of protons into environment is either very fast (type-I and II liquids) or completely blocked (type-III liquids). The proton exchange rate at the surface-air interface is expected to fall in the middle zone, and thus may lead to the $\sim 1 \mathrm{hr}$ intermediate conductance decay time typically observed in the air (Fig.6.10a). Same model can also explain the previously reported indefinitely long lifetime of c-AFM created interface conductance in vacuum or at low temperatures $[60,74,167,188]$. As molecular water layers desorb in vacuum, the surface proton dissociation path- 
way through hydrogen bonds is eliminated. The reduced temperature on the other hand can suppress the thermally activated hopping process. As a result, the c-AFM created conducting patterns are highly stable in vacuum or cryogenic environments.

Based on this model, now we can understand why oxygen plasma and c-AFM can both change the LAO/STO interface electrical properties but the resultant effects have very different persistence in the air. Considering only the dissociated water layer, surface $\mathrm{Al}-\mathrm{OH}$ bonds and $\mathrm{O}-\mathrm{H}$ bonds have very similar binding energies. However, with the additional molecular water layers, proton dissociation barriers are substantially weakened while the surface $\mathrm{OH}$ still remain strongly bonded. In LAO/STO heterostructures with initially metallic interfaces $\left(d_{L A O}>3 \mathrm{uc}\right)$, the reduction of interface conductance by oxygen plasma is related to the increase of the surface $\mathrm{OH}$ density. The $\mathrm{Al}-\mathrm{OH}$ bonds produced in this process are very rigid at room temperature, which thus lead to a long-living variation in the interface conductance. In contrast, the interface metallicity induced in initially insulating 3 uc LAO/STO samples by positively biased c-AFM scan is due to the field driven surface protonation process. When the probe field is withdrawn, the proton hopping exchanges between the LAO surface and the molecular water layers are still very active, which leads to the interface's faster recovery to thermal equilibrium insulating state.

\subsection{Summary}

Surface dangling bonds as well as adsorbates formed from water in the air are important factors to consider in $\mathrm{LaAlO}_{3} / \mathrm{SrTiO}_{3}$ heterostructure. Strong correlations between the interface density of states and the dissociative adsorption of water at $\mathrm{LaAlO}_{3}$ surfaces were observed. Changing the densities and distributions of surface protons and hydroxides were found to allow effective tuning of the interface metallic- 
ity. In heterostructures with different $\mathrm{LaAlO}_{3}$ layer thicknesses, such extrinsic effects are always present but the exact level of changes induced may vary. Roles of charge transfer, electron correlations and lattice distortions were discussed to help understand the observed phenomena. Both surface protons and hydroxides are strongly bonded at low surface water coverages. However, with the multiple molecular water layers typically formed in the air, the stability of $\mathrm{Al}-\mathrm{OH}$ bonds and $\mathrm{O}-\mathrm{H}$ bonds become very different. Long lasting production of high densities surface hydroxides can be achieved by oxygen plasma, which results in the suppression of interface conductance. In 5 uc LAO/STO, metal-insulator transition due to the oxygen plasma treatment were observed at low temperatures. Bridged by hydrogen bonds, proton adsorption and desorption are active at room temperature. Using a simple thermodynamic model based on the thermally activated proton transfers between the LAO surface and water molecules, the effects of previously reported c-AFM lithography and the decay rates of interface conductance in different environments were well explained. Surface water adsorptions are commonly observed at oxides or semiconductors surfaces. We expect that the results presented here may also be of value to the study of other low dimensional material systems. 


\section{Chapter 7}

\section{Summary}

As a part of the research for the dissertation, an ultrafast Ti:Sapphire laser system was built. Once the laser was made stable and noise free, characterization revealed that the pulsewidth of the laser is $30 \mathrm{fs}$, bandwidth is $35 \mathrm{~nm}$, and the repetition rate is $74 \mathrm{MHz}$. Futhermore, the center wavelength of the laser is tunable from 800 $\mathrm{nm}$ to $840 \mathrm{~nm}$. This home built laser is used to pump a BBO crystal which does SHG. The output of the BBO and the laser is used for pump probe spectroscopy. The pump probe spectroscopy system is fitted with cryostation so that the measurements can be done at different temperatures. The cryostation has electrical measurement capability. So, the system can be adapted to do regular photocurrent measurements. Using this system, ultrafast carrier dynamics and photocurrent on $\mathrm{SmB}_{6}$ was studied.

The pump-probe spectroscopy measures a coherent picture of the band structure evolvement in $\mathrm{SmB}_{6}$ with change in temperature. Two characteristic temperatures $T_{1}=100 \mathrm{~K}$ and $T_{2}=20 \mathrm{~K}$ are revealed. $T_{1}$ corresponds to the opening of $f$ - $d$ hybridization gap which is pin-pointed by the abrupt disappearance of $f$-band bulk plasmon. In between $T_{1}$ and $T_{2}$, the slow relaxation dynamics is attributed to "phonon bottleneck" which are common in this kind of systems. Below $T_{2}$, the dramatically 
accelerated carrier relaxation is seen. The accelerated relaxation is explained by the emergence of surface in-gap state which assists in carrier recombination process through low energy acoustic phonons. The negative signal in PI $\Delta R / R$ is attributed to surface lattice heating. Measurement results obtained in single crystal and thin film samples qualitatively agree with each other, supporting the existence of a possible surface conducting layer at low temperature. Furthermore, the optical measurement is consistent with the transport measurement which reveals the supposedly "surface state" at very low temperature.

However, more studies are necessary to conclude whether the observed in-gap states are indeed topological surface states. For example, it will be very interesting to look for plasmon modes associated with these in-gap states. The plasmon dispersion relation and damping properties can provide highly valuable insight into the 'ingap states' dispersion and their correlations with the bulk. Also, time resolved measurements of the spin dynamics can be performed to evaluate the spin-orbit coupling effect and other spin related features predicted by TKI theory. Furthermore, if $\mathrm{SmB}_{6}$ is proved to be a true 3D topological insulator then heterostructure of $\mathrm{SmB}_{6}$ with a ferromagnetic material can be made which can be used to generate Majorana fermion.

From the circular photogalvanic effect (CPGE) measurement, helicity dependent photovoltage is observed in $65 \mathrm{~nm}$ thick $\mathrm{SmB}_{6}$ film grown on MgO. CPGE measurements on this sample provided two possibilities for the origination of CPGE; topological helical Dirac fermions on the surface of the $\mathrm{SmB}_{6}$ and simple outcome of Rashba type spin-orbit coupling from the surface. In case of topological surface state, carrier spin and momentum are always orthogonal to one another. When circular polarized light is absorbed, the spin angular momentum of the light is transferred to 
the surface electron spin. Depending on the helicity of the light, electron with certain spin can be selectively excited. Consequently, the drift of the carriers produces CPGE. Measurement on thinner sample revealed that CPGE is absent which contradicts that the CPGE could be from the SOI. Furthermore, the absence of CPGE can be explained by inter surface coupling between top and bottom topological surface of the thinner film. Moreover, the magnitude of the PV decreases with increase in the temperature. In future it will be very interesting to measure the time resolved spin dynamics with ultrafast magneto optics Kerr effect.

For the 2nd 2D system (LAO/STO interface) the roles of surface adsorbents on the interface conductivity is examined. From the experiment and theoretical modeling it can be concluded that "polar catastrophe" model can explain the interface conductivity due to surface adsorbates. Adsorbates formed from dissociation of water in the air, hydrogen bonds, and surface dangling bonds are very important factors to consider in LAO/STO heterointerface conductivity. Strong correlations between the interface density of states and the dissociative adsorption of water at LAO surfaces is observed. Changing the densities and distributions of surface protons and hydroxides allows effective tuning of the interface metallicity for both 3 uc and 5 uc thick LAO on STO. The always present 2DEG in sample with 3+ uc LAO layer thickness is also supported by the DFT model. Roles of charge transfer, electron correlations, and lattice distortions are discussed to help understand the observed phenomena. Both surface protons and hydroxides are strongly bonded at low surface water coverage. However, with the multiple molecular water layers typically formed in the air, the stability of Al-OH bonds and $\mathrm{O}-\mathrm{H}$ bonds become very different. Furthermore, the transport measurements and XPS spectra reveals that long lasting production of high densities surface hydroxides can be achieved by oxygen 
plasma. This results in the suppression of interface conductance. In 5 uc LAO/STO, metal-insulator transition due to the oxygen plasma treatment is observed at low temperatures. Bridged by hydrogen bonds, proton adsorption and desorption are active at room temperature. Using a simple thermodynamic model based on the thermally activated proton transfer between the LAO surface and water molecules, the effects of previously reported c-AFM lithography and the decay rates of interface conductance in different environments are well explained. Surface water adsorptions are commonly observed at oxides or semiconductors surfaces. The results presented here may also be of value for studying other low dimensional material systems. In future it will be interesting to selectively engineer different interface structures by oxygen plasma cleaning for device application or understanding the fundamentals. 


\section{Appendix A}

\section{Cavity Alignment}

Alignment of the intercavity optics is very important for optimal operation of a modelocked Ti:Sapphire laser. This appendix discuses the intercavity alignment of the optics. Given below are the itemized alignment procedures.

1. The Ti:Sapphire crystal is placed in a holder, which is mounted in a XY and rotation stage. The height of the crystal is fixed and it determines the height of all the optics in the cavity. Thus, the height of the irises to be used for the alignment should be fixed to the height of the crystal. For initial alignment, only $1 \mathrm{~mW}$ pump power is enough. Height of the input pump should be fixed, using a steering dielectric mirror (SM1), to the center of the crystal with iris (I1) as shown in figure A.1. Iris (I2) should be placed far away from I1 and dielectric mirror (SM2)should be steered to make sure the height is aligned. Beam walking should be performed until pump goes through both of the iris without clipping. 


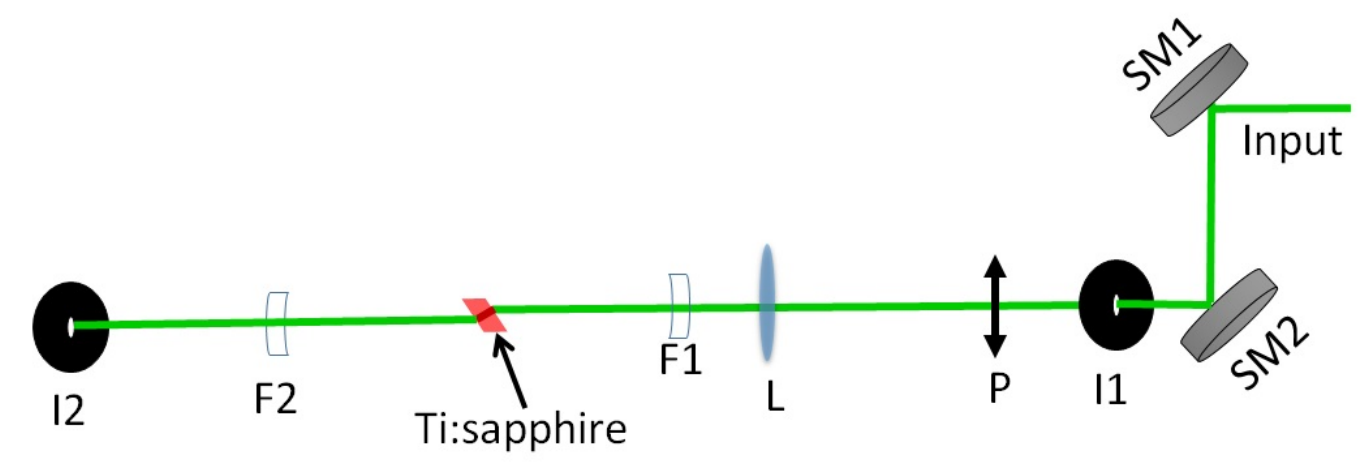

Figure A.1: A schematic for pump alignment

2. Once the height of the pump is fixed, the polarizer $(\mathrm{P})$ and lens $(\mathrm{L})$ can be inserted into the beam path. The focal length of the lens is $150 \mathrm{~mm}$. The polarizer and lens should have proper coatings for 533nm (pump wavelength). The lens is mounted on a translation stage in such a way that the principle axis of the lens, light path, and translation stage direction are co-linear, with the light going through the center of the lens.

3. Now, the Ti:Sapphire crystal can be inserted in the light path roughly at the focal point of the lens. The incident angle of the pump beam on the Ti:Sapphire crystal should be at the Brewster angle so that the reflection from the front surface of the Ti:Sapphire crystal can be minimized. In order to achieve the Brewster angle, the crystal can be rotated using the rotation mount.

4. The two focusing mirrors (F1 and F2) can be inserted in to the path. The inner center of the focusing mirror should be roughly the center of the pump. The angle of the two focusing mirrors with respect to the pump path is discussed earlier (see table 2.1).

5. Now, the mirror M3, M4, and HR can be placed. The length of the path 
between each optics and the angle in this arm has been discussed earlier (see table2.1). The same iris should be used to check the height of the beam between F2-M3, M3-M4, and M4-HR. With only few mW of pump, the luminescence from the Ti:Sapphire crystal is very low. The reflected green light is ideal for alignment purpose. This green light should hit the center of each optics after the focusing mirror. Using the same iris the reflection from the HR should follow the same path back to the F2 as shown in the figure A.2.

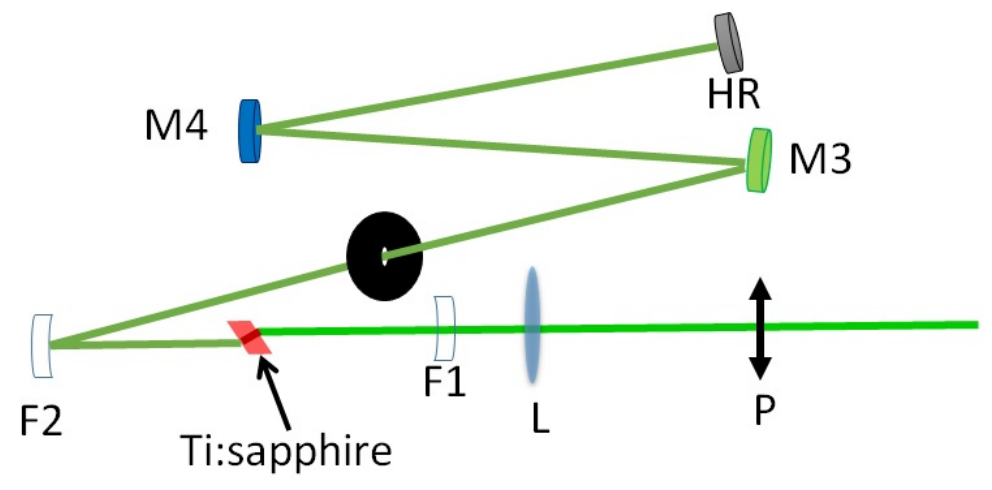

Figure A.2: A schematic for alignment of one arm

6. After aligning the optics for the first arm, the other arm F1-M1-M2-OC can be aligned. Path length and the angle between each of the optics is provided earlier (see table 2.1). In this arm, we depend on the luminescence from the Ti:Sapphire crystal to do the alignment. For this reason, the power of the input pump should be increased to $2 \mathrm{~W}$. Proper eye protection is a must for this step. The room light should be turned off in order to see the luminescence easily. Using IR viewer and optics wipes the luminescence can be tracked. This luminescence should be steered to hit the center of each optics all the way to the OC. Reflection from the OC should roughly trace back to the same path. Once this is achieved, the pump power can be increased to $6.5 \mathrm{~W}$ and 
a power detector sensitive upto $\mu \mathrm{W}$ level can be placed behind the OC. Now, the detector should read few $\mu \mathrm{W}$ of power. The $\mathrm{OC}$ can be steered gently so that the detector reads more output power. At some point, the detector should show sudden jump in power (few $100 \mathrm{~mW}$ ). At this point, the cavity is lasing. Using the IR viewer one can see bright spot on each optics. Using the combination of the $\mathrm{HR}$ and $\mathrm{OC}$, the output power should be maximized. The laser spot on each optics should be walked to the center of each mirrors. Also, the focusing mirror, Ti:Sapphire crystal, and the lens can be tuned to maximize the output power. Maximum output power corresponds to $T E M_{00}$ mode. $T E M_{00}$ mode can be verified by using an objective to expand the beam waist. If the beam waist after the expansion is still round, the mode of the laser is close to $T E M_{00}$. With $6.5 \mathrm{~W}$ of pump, the laser should output around $1200 \mathrm{~mW}$ of CW light.

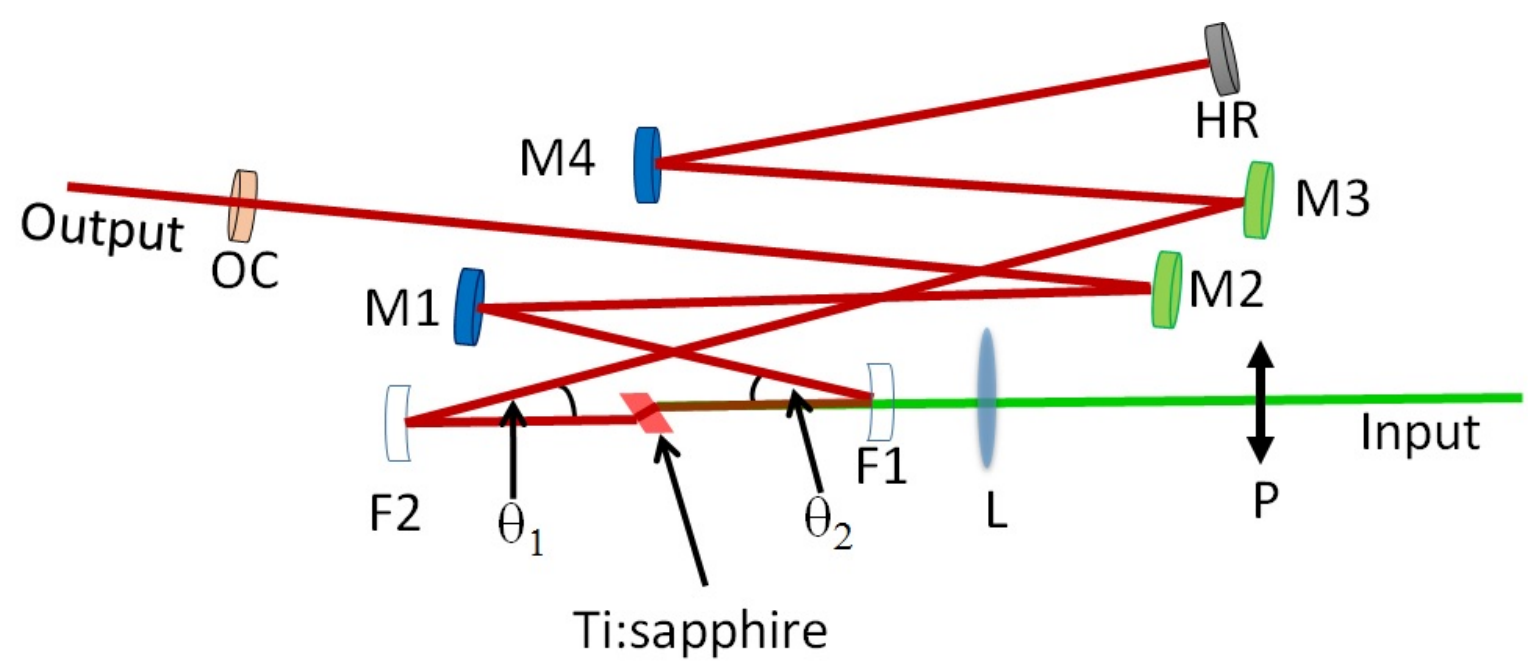

Figure A.3: A schematic for cavity alignment

7. The next step after optimizing the CW output is the mode locking of the laser.In order to initiate the modelocking, the cavity must be disturbed. One 
can do that by pushing F2 a little and then release it. One needs to observe the spectrum of the laser output to confirm the modelocking. If the spectrum of the laser gets wider with F2 being pushed, the laser is trying to modelock.

8. Using a fast photodetector connected to oscilloscope the pulse and power meter the pulsetrain stability can be monitored. Spectrometer can give information on bandwidth and corresponding pulsewidth. Cavity optices can be tuned with micrometer associated with each optics until desirable result is achieved. 


\section{Appendix B}

\section{Troubleshooting of the Laser}

\section{Oscillator}

Modelocking, an essential outcome of a Ti:Sapphire laser, can be lost abruptly or over the time. This appendix discuses how to troubleshoot the laser cavity to get

back the pulsed output. Listed below are the steps one should follow to troubleshoot the oscillator:

1. It has been observed that the change in room temperature and humidity adversely affects the cavity alignment and stability of laser. If the laser is not modelocking, the first order of operation is to check for the change in the temperature or humidity of the room before opening the cavity and aligning the optics.

2. If modelocking is gone abruptly due to sudden vibration to the table (e.g. an optical post holder slipping from hand knocking on the optical table), pushing one of the cavity concave mirrors (as mentioned in Appendix A) should fix it.

3. If the laser output is slowly decreasing over the time, the optics (surface of 
mirrors, OC, HR, Ti:Sapphire crystal) might have gone dirty. All the optics should be properly wiped with methanol. If the optics are still dirty they can be wiped by acetone followed by methanol. The output power should go back to same as before.

4. Back reflection to the cavity from the optics outside the cavity can destroy the crystal in long run and stability of laser. The optics outside the cavity should be aligned in such a way that the light has small angle to the front surface of the optics instead of normal incidence. This can avoid back reflection to the cavity.

5. If the laser output is noisy and all the previous steps cannot fix it. The noise could also be coming from the pump laser. All the commercial lasers needs to be tuned periodically. 


\section{Appendix $\mathrm{C}$}

\section{Second Harmonic FROG}

FROG as a method for characterizing an optical pulse was invented by Rich Terbino [189]. In FROG, the pulse that needs to be characterized is gated by another ultrashort pulse called gate pulse. In second harmonic FROG (SH-FROG), similar to autocorrelation technique, the pulses are crossed in a medium with an instantaneous second order nonlinearity $\left(\chi^{2}\right)$. The output pulse (from this nonlinear crystal) is the convolution of unknown electric field $(E(t))$ of the input pulse, that needs to be characterized, with the gating field $(g(t))$ (with itself). In autocorrelation, the emitted nonlinear output is sent to a slow photo detector. Hence, an autocorrelator measures only in time domain. In SG-FROG, the output is sent to a spectrometer. So, SG-FROG is an autocorrelation type measurements in which the signal is spectrally and temporally resolved. Intensity of this output is called spectrogram. Instead of measuring signal vs. delay, one measures signal spectrum vs. delay. Figure C.1 is a setup for measuring SH-FROG. It looks similar to an intensity autocorrelation setup, except the photodetector is replaced by a spectrometer. 


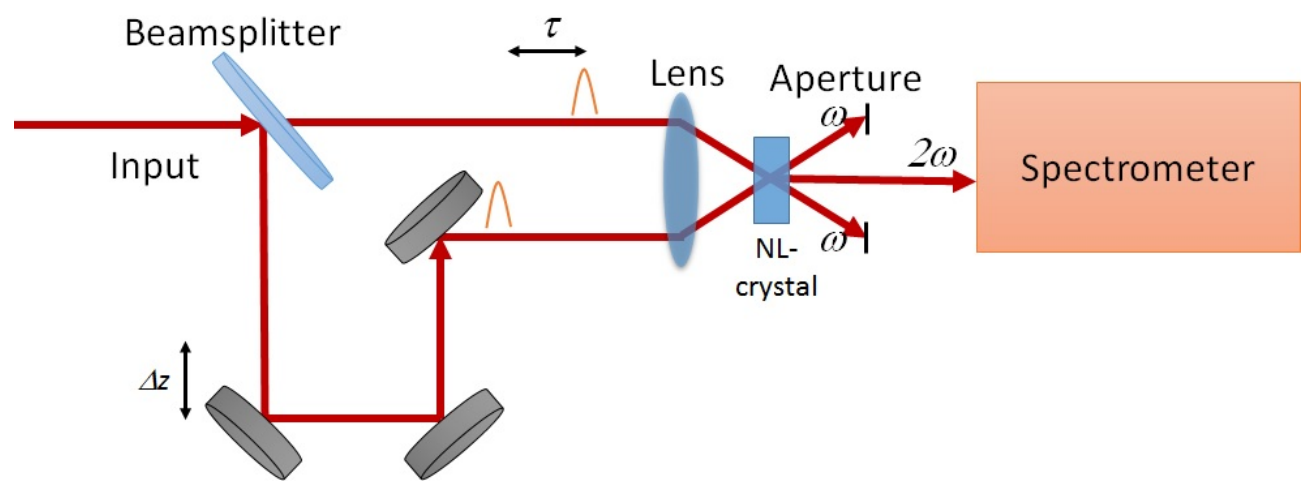

Figure C.1: A SH-FROG setup.

The intensity of the spectrogram is given by:

$$
I_{F R O G}(\omega, \tau)=\left|\int_{-\infty}^{\infty} E(t) g(t-\tau) e^{(-i \omega t)} d t\right|^{2}
$$

In the SH-FROG, the gating pulse is the copy of the pulse to be measured. $g(t) \equiv E(t)$.

$$
I_{F R O G}(\omega, \tau)=\left|\int_{-\infty}^{\infty} E(t) E(t-\tau) e^{(-i \omega t)} d t\right|^{2}
$$

FROG algorithm is based on iterative-Fourier-transform algorithm. Detail working mechanism of a FROG is shown in figure C.1.

$$
E_{s i g}(\omega, \tau)=E(t) E(t-\tau)
$$




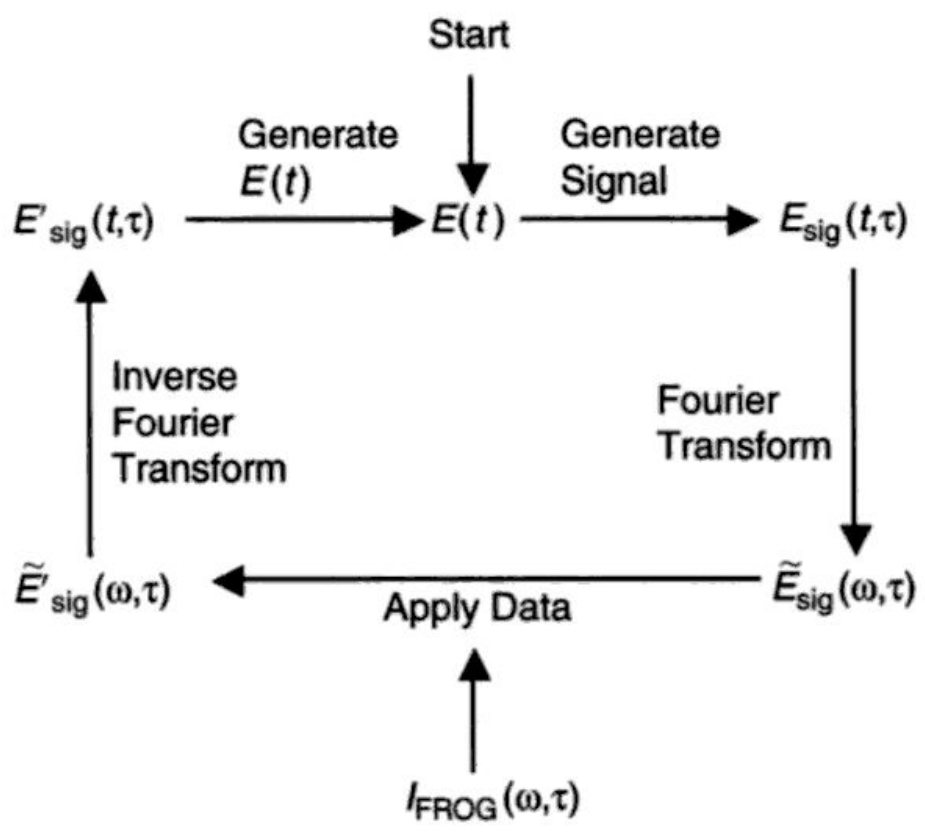

Figure C.2: Schematic diagram of a FROG algorithm [189].

First, it guesses the initial electric field $E(t)$ then a signal field $E_{\text {sig }}(\omega, \tau)$ is generated. This field is then Fourier transformed with respect to $t$ in order to generate the signal field $\widetilde{E}_{s i g}(\omega, \tau)$ in the frequency domain. The measured FROG trace $I_{F R O G}(\omega, \tau)$ is then used to generate an improved signal field $\widetilde{E}_{s i g}^{\prime}(\omega, \tau)$. Since the squared magnitude of $\widetilde{E}_{s i g}^{\prime}(\omega, \tau)=I_{F R O G}(\omega, \tau)$, this step nearly always involves replacing the magnitude of $\widetilde{E}_{\text {sig }}(\omega, \tau)$ with the square root of the measured trace to generate $\widetilde{E}_{\text {sig }}^{\prime}(\omega, \tau)[189] . \widetilde{E}_{\text {sig }}^{\prime}(\omega, \tau)$ is then transformed back into the time domain by applying an inverse Fourrier transform. Finally, the modified signal field $\widetilde{E}_{s i g}^{\prime}(t, \tau)$ is used to generate a new guess for $E(t)$. The iteration is then repeated until the algorithm generates a better guess, which eventually approaches the correct complex electric field. 


\section{Appendix D}

\section{Pump-probe Setup}

\section{D.1 Second Harmonic Generation}

Crystal lacking inversion symmetry can exhibit second order non-linearity. This can give rise to a non-linear phenomenon of frequency doubling [102], where an input (pump) laser generates another laser with twice the frequency (i.e. half the wavelength). This process is also called second-harmonic generation (SHG). In the experimental setup, the output of the Ti:Sapphire is focused on the Barium Betaborate $(\mathrm{BBO})$ crystal using a concave mirror with the focal length of $100 \mathrm{~cm}$. The signal and idler is collimated back using another $100 \mathrm{~cm}$ focal length concave mirror. Figure D.1 shows the SHG by a BBO crystal followed by the separation of the residual pump and second harmonics by a dichroic mirror.

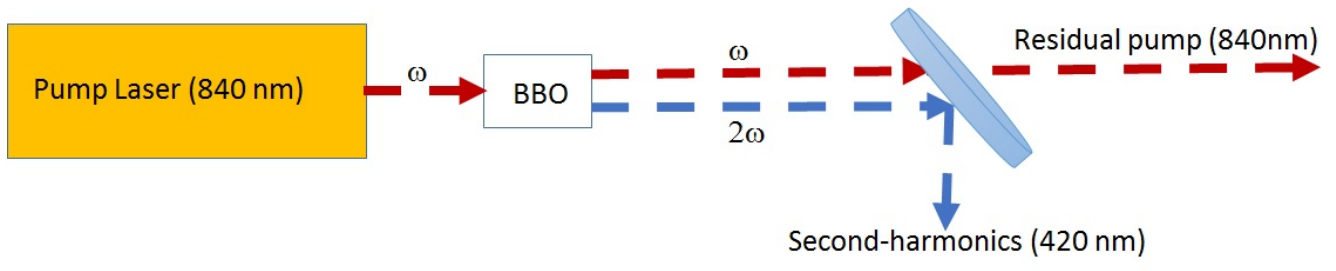

Figure D.1: SHG by a BBO crystal 


\section{D.1.1 Theory of SHG}

In order to understand the theory behind SHG it is very important to understand the basics of non-linear optics [190]. In a conventional (linear) optics, the induced polarization $P$ depends linearly in the electric field $E$ which can be described by the relationship:

$$
P(t)=\epsilon_{0} \chi^{(1)} E(t)
$$

Here, $\chi^{(1)}$ is the $1^{\text {st }}$ order susceptibility of the medium and $\epsilon_{(0)}$ is the permittivity of the free space. However, at very high light intensities, such as those provided by a pulsed lasers, the dielectric polarization $(P)$ responds nonlinearly to the electric field $(E)$ :

$$
P(t)=\epsilon_{0}\left(\chi^{(1)} E(t)+\chi^{(2)} E^{2}+\chi^{(3)} E^{3}+\ldots . .\right)
$$

$\chi^{(2)}$ and $\chi^{(3)}$ are the 2-nd and 3-rd order susceptibilities. If we only consider the second order nonlinearity, then the polarization $(P)$ becomes

$$
P^{(2)}=\epsilon_{0} \chi^{(2)} E^{2}(t)
$$

If we assume $E(t)$ is made of two simple sinusoidal components with frequencies $\omega_{1}$ and $\omega_{2}$, this $E$ can be rewritten as

$$
E(t)=E_{1} \cos \left(\omega_{1} t\right)+E_{2} \cos \left(\omega_{2} t\right)
$$

After using Euler's relation

$$
E(t)=\frac{1}{2} E_{1} e^{-i \omega_{1} t}+\frac{1}{2} E_{2} e^{-i \omega_{2} t}+\text { c.c. }
$$


where c.c. is the complex conjugate. Plugging this equation for $P^{(N L)}$ gives

$$
\begin{array}{r}
P^{N L}=\epsilon_{0} \chi^{(2)} E^{2}(t)=\frac{\epsilon_{0}}{4} \chi^{(2)}\left[\left|E_{1}\right|^{2} e^{-i 2 \omega_{1} t}+\left|E_{2}\right|^{2} e^{-i 2 \omega_{2} t}\right. \\
+2 E_{1} E_{2} e^{-i\left(\omega_{1}+\omega_{2}\right) t} \\
+2 E_{1} E_{2}^{*} e^{-i\left(\omega_{1}-\omega_{2}\right) t} \\
\left.+\left(\left|E_{1}\right|^{2}+\left|E_{2}\right|^{2}\right)+\text { c.c. }\right]
\end{array}
$$

In above equation, the non-linear polarization with $2 \omega_{1}$ and $2 \omega_{2}$ corresponds to the SHG. Energy level diagram for SHG is given below in figure D.2.

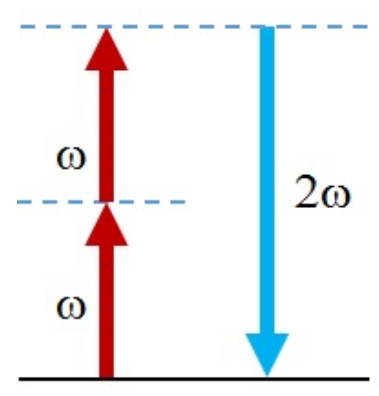

Figure D.2: Energy-level diagram describing SGH

\section{D.2 Delay}

A delay is introduced between pump and probe with the help of a translation stage in order to resolve the signal temporally. The equation given below describes the temporal separation between pump and probe pulse $(\tau)$ as a function of displacement of the delay $(d)$ :

$$
\tau=\frac{2 d}{c}
$$


where, $c$ is the speed of light. many commercial micrometer can easily get sub-micron resolution. Hence, femtosecond delay between pump and probe is easily achieved.

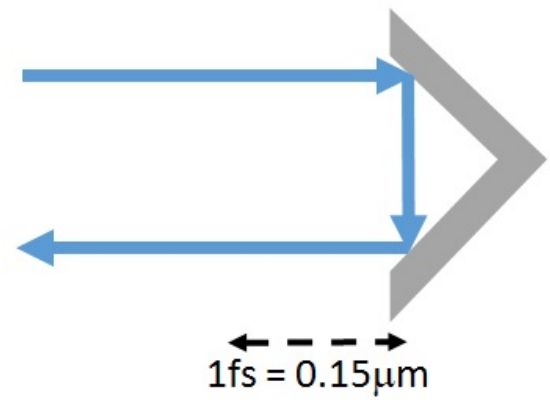

Figure D.3: A schematic diagram showing delay due to the displacement of the translation stage.

\section{D.3 Modulation}

The amplitude modulation of the pump is done by combination of a PEM and polarizer. Hinds PEM is used for the fast modulation of light. According to Hinds PEM mannual, a transparent fused silica is stressed by compressing or stretching. Since the material is birefringent, the refractive index can be changed just by compressing or straching which results in different linear polarizations of light to have different speeds. Which means, when the fused silica is compressed, the polarization component parallel to the principle axis travels faster than the vertical component. The magnitude of the birefringence is controlled electronically with a PEM Controller. The phase difference between the components at any instant of time is called the retardation or retardance [191]. The peak retardation is the amplitude of the sinusoidal retardation as a function of time. 


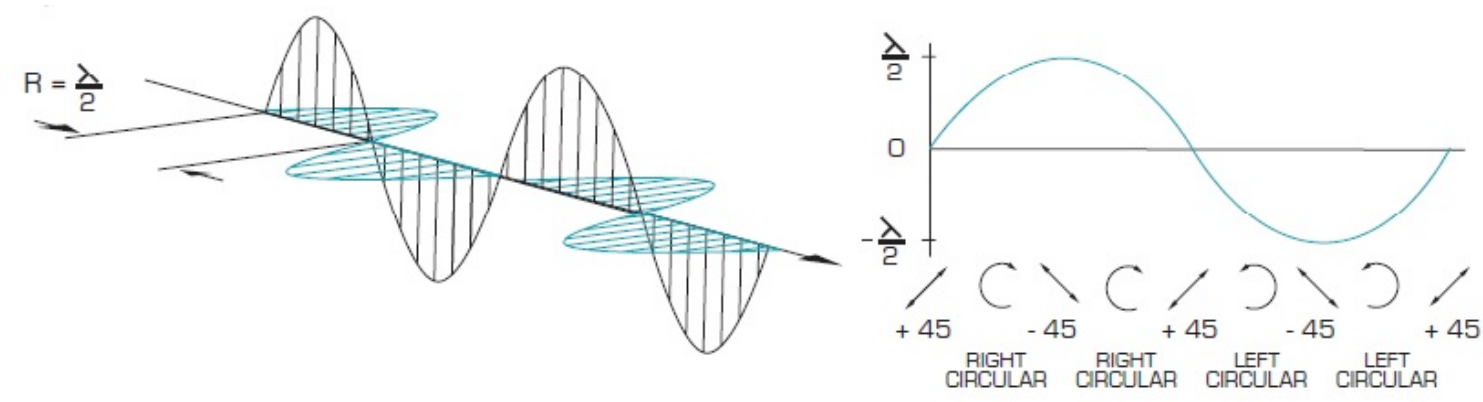

Figure D.4: The figure on the left shows peak retardation at one half of the wavelength of the light and the figure on the right shows retardation vs. time with a PEM [191].

When the peak retardation reaches one-half the PEM acts as a half wave plate with change in polarization from vertical to horizontal at $84 \mathrm{kHz}$. A polarizer is placed after the PEM which either blocks the light if the polarization axis is perpendicular to the light polarization or allows the light if the polarization of the light is parallel the polarization axis. The combination of the PEM and polarizer acts as a high frequency modulator for maximum noise cancellation. More discussion on on the PEM can be found in the manual [191].

\section{D.4 Lock-In Amplifier}

A lock-in amplifier is a type of amplifier that can extract a signal, as defined by the reference, from an extremely noisy environment. The lock-in amplifier takes the input signal (from the detector as a sinusoidal voltage due to modulation by PEM), multiplies it by the reference signal (from the PEM), and integrates it over a specified time (also called time constant). The resulting signal is a DC signal, where the contribution from any other signal that does not have the same frequency as the reference signal is attenuated close to zero. Furthermore, the out-of-phase component of this signal that has the same frequency as the reference signal is also attenuated 
(because sine function is orthogonal to a cosine function of the same frequency). This makes a lock-in amplifier a phase-sensitive detector. The analog output signal (voltage) from the amplifier is feed into a data acquisition card connected to a computer. The data is acquired and processed using LabView.

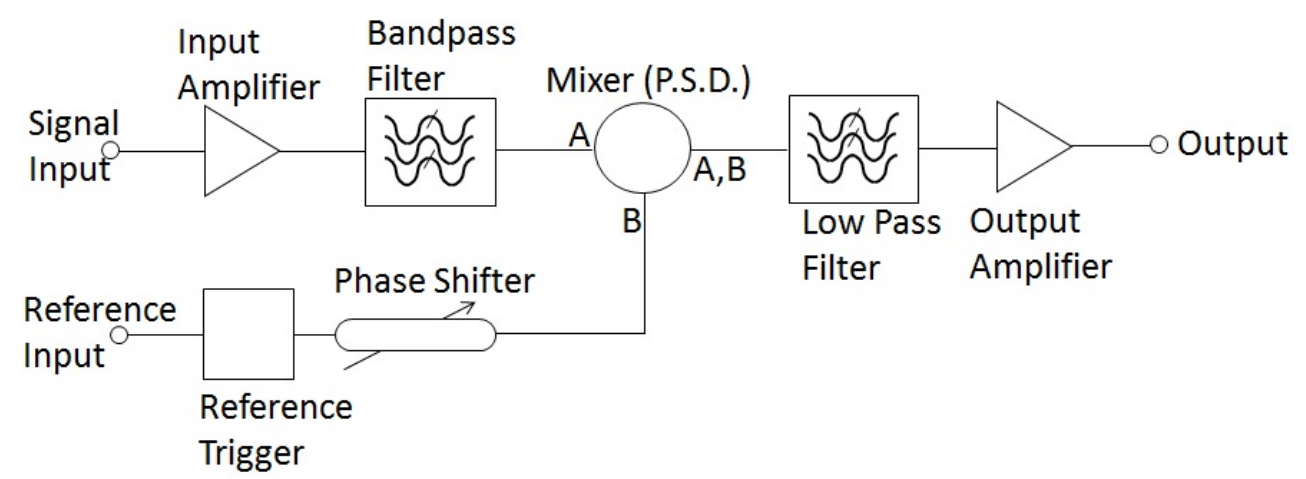

Figure D.5: A schematic diagram showing the working mechanism of a lock-in amplifier [192].

\section{D.5 Detector}

Signal (reflected light) is collected and sent to a Nirvana autobalance detector. This autobalance detector is based on auto-balancing subtraction circuits design by Phil Hobbs [193]. The detector consists of two photodiodes, one for signal and one for reference. The reflected light from the sample is sent to the signal photodiode and small sample of oscillator output is sent to the reference photodiode. Other components inside the detector are: a current splitter, a current subtraction node, a transresistance amplifier, and a feedback amplifier which are based on the subtraction of the photocurrents from reversed biased visible and near-infrared photodiodes [194]. The output of the photodetector $(A)$ can be expressed as $A=(I S-g \times I R) \times R f$. 


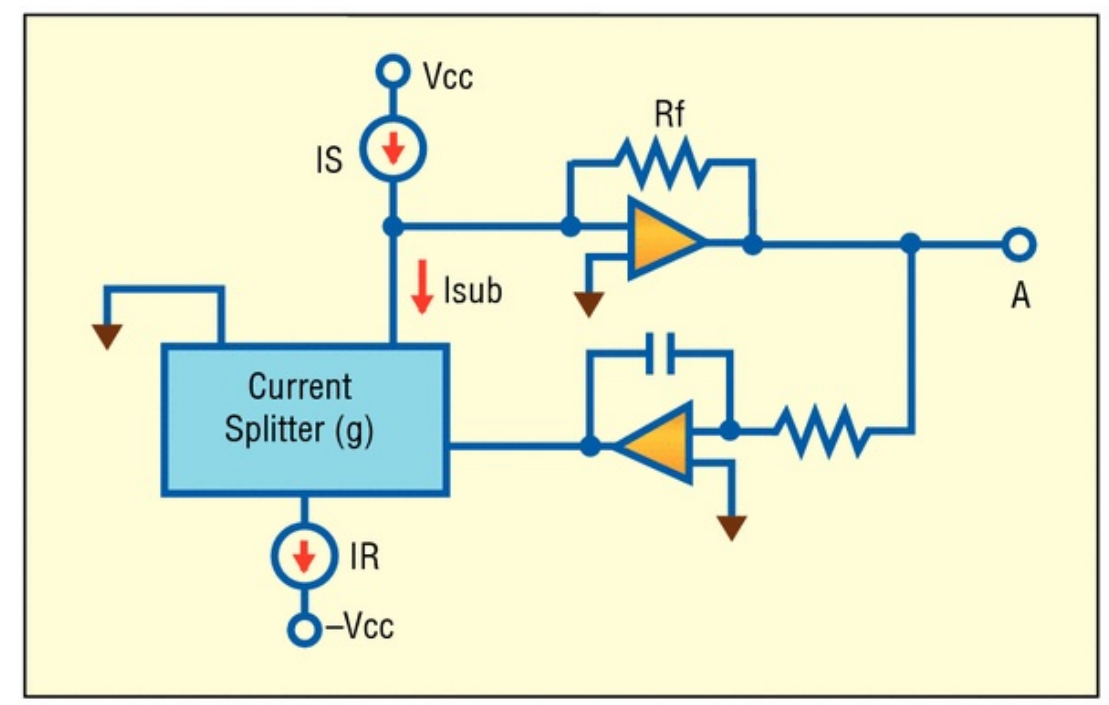

Figure D.6: Feedback loop in an autobalance detector. This image is acquired from application notes for Nirvana balance detector from Newport website

In figure D.6, IS is the signal photodiode current, $I R$ is the reference photodiode current, $R f$ is the value of the feedback resistor, and $g$ is the current-splitting ratio. $g$ describes how much of the reference current comes from the subtraction node (Isub) and how much comes from ground. In signal mode, $g$ is zero therefore no reference photocurrent comes from the subtraction node. Here, the output $A$ is simply an ampli?ed version of the signal current. The detector can cancel commonmode laser noise with greater than 50-dB rejection at frequencies less than $125 \mathrm{kHz}$. With this balance detection scheme the noise coming from the oscillator can be drastically reduced. Output $(A)$ from the detector is feed into a Lock-in amplifier. More discussions on the detailed working mechanisms can be found on the manual [194]. 


\section{Appendix E}

\section{Hall Effect Measurement}

Hall effect measurement is done to characterize the transport properties of the material. From this measurement, the information on resistivity, charge carrier density, and mobility can be obtained. Quantum Design (P)hysical (P)roperty (M)easurement (S)ystem is used for Hall effect measurement as a function of temperature.

\section{E.1 Hall Bar Configuration}

When a current (consists of moving charge carriers like electron, holes, etc) carrying material is placed in a magnetic field the carriers experiences a force, called the Lorentz force. Due to this force, the carriers cannot move in a straight path (Figure E.1). 


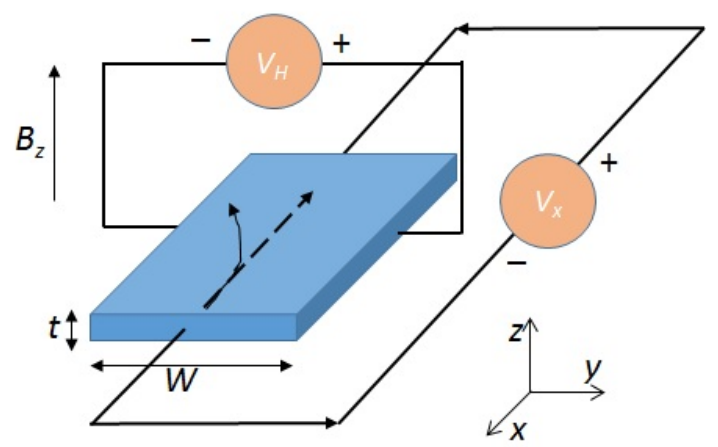

Figure E.1: A Hall bar configuration for Hall effect measurement.

When carrier are moving in a direction perpendicular to the applied magnetic field (Figure E.1), it experiences a magnetic force $(-q v \times B)$ perpendicular to both directions, given by right hand rule. The resulting Lorentz force $\mathrm{F}$ is therefore equal to $-q(E+v \times B)$, where $\mathrm{q}\left(1.602 \times 10^{-19} \mathrm{C}\right)$ is the elementary charge, $E$ is the electric field, $v$ is the particle's drift velocity, and $B$ is the magnetic field. In a steady state, the magnetic force is balanced by the electric force. Mathematically,

$$
q E=q v B
$$

For simplification lets consider an n-doped, bar-shaped semiconductor in which the carriers are electrons with bulk density $(n)$. Due to the Lorentz force, the electrons drift away from the current direction and accumulate in one side of the bar. Holes will be accumulated in the other side. The separation of the positive and negative charge results in the Hall voltage, a potential drop across the two sides of the sample. This Hall voltage can be measured as shown in figure E.1. The Hall voltage is given by:

$$
V_{H}=\frac{I B}{q n w}
$$

where, $I$ is the current and $w$ is the sample thickness. Thus, by measuring the Hall 
voltage one can measure charge carrier density given by:

$$
n=\frac{I B w}{q V_{H}}
$$

The Hall voltage will be negative for n-doped semiconductor and positive for p-doped semiconductor. Also, the resistivity $(\rho)$ can be easily calculated from the dimension and the resistance of the material. Since, the resistivity involves both carrier density and mobility, the Hall mobility can be extracted using the equation:

$$
\mu=\frac{\left|V_{H}\right| w}{\rho I B}=\frac{1}{q n \rho}
$$

Hence, a careful Hall effect measurement can provide information on resistivity, charge carrier density, and mobility.

\section{E.2 Van der Pauw Configuration}

A regular Hall effect measurement is useful for characterizing a bulk material. More often than not the samples are in thin film form. These thin films are usually grown on top of a substrate. In these situation, van der Pauw is the choice for measuring sheet resistance $\left(R_{S}=\rho / d\right)$, sheet density $\left(n_{S}=n d\right)$, and mobility. The schematic diagram for measuring $R_{S}$ is given in figure E.2. 1,2,3, and 4 are the contact point on the sample surface for measuring voltage and sourcing current. 

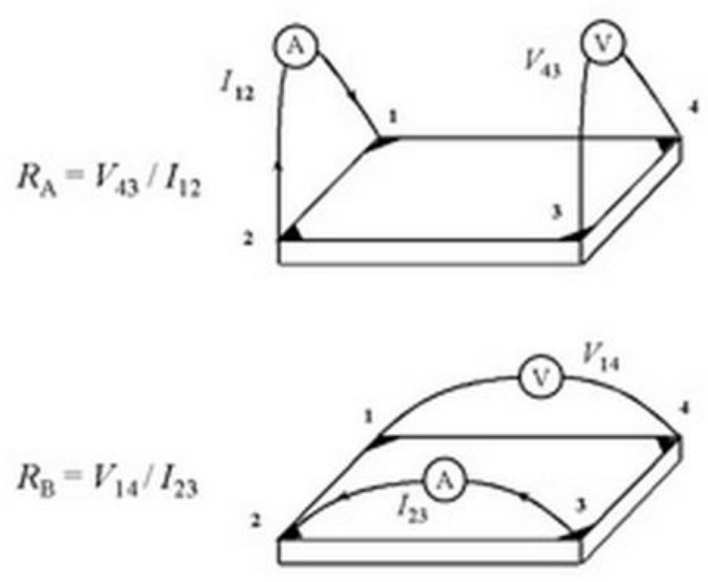

Figure E.2: A van der Pauw configuration for measuring sheet resistance [195].

The $R_{A}$ and $R_{B}$ are the two resistace measured as shown in diagram. They are related to $R_{S}$ by the van der Pauw equation:

$$
\exp \left[\frac{-\pi R_{A}}{R_{S}}\right]+\exp \left[\frac{-\pi R_{B}}{R_{S}}\right]
$$

This equation can be numerically solved to extract $R_{S}$. In order to extract mobility and sheet density Hall measurement is done in the thin film. The schematic diagram for Hall measurement in van der Pauw configuration is given in figure E.3. In this configuration, the Hall voltage is measured perpendicular to the sourced current direction, as explained in previous section. 


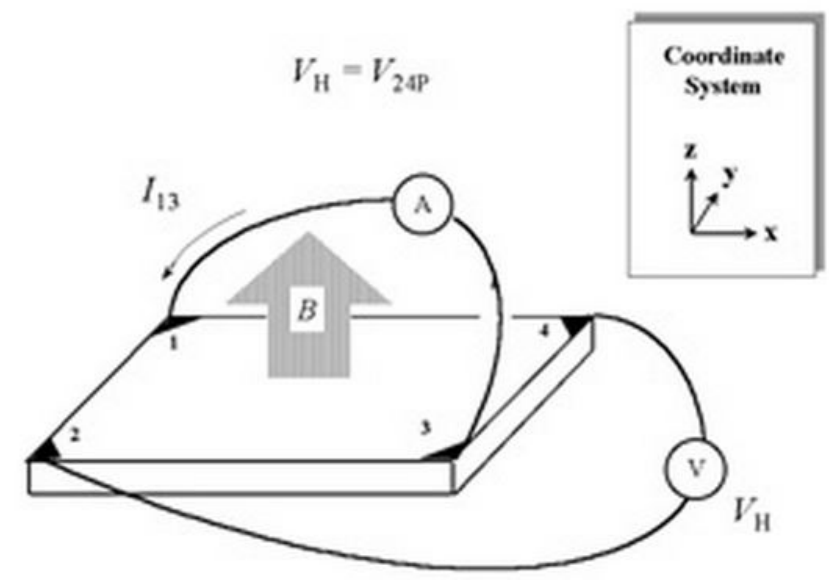

Figure E.3: A van der Pauw configuration for Hall measurement [195].

Once the Hall voltage is measured, the $n_{S}$ can be calculated using the equation:

$$
n_{S}=\frac{I B}{q\left|V_{H}\right|}
$$

$I, B$, and $q$ are all known values. Mobility is given by $\left|V_{H}\right| / R_{S} I B$.

Thus, Hall effect measurement gives information on reistivity, charge carrier density, and mobility that are essential for characterizing a material. 


\section{Appendix F}

\section{Sample Processing}

Samples for the experiments are acquired from the collaborators. The samples are then processed in cleanroom environment where metal contacts are deposited for

electrical and electro-optical measurements. Also, sample surface can be modified using oxygen plasma cleaning, ion etching, solvent deposition, etc.

\section{F.1 Photolithography}

Photolithography is used for making pattern structure on the top surface of the sample. Once the pattern is developed on the sample surface, either other material can be grown top of it or the material can be selectively etched in the exposed surface. A step by step process for photolithography is given in figure F.1. 


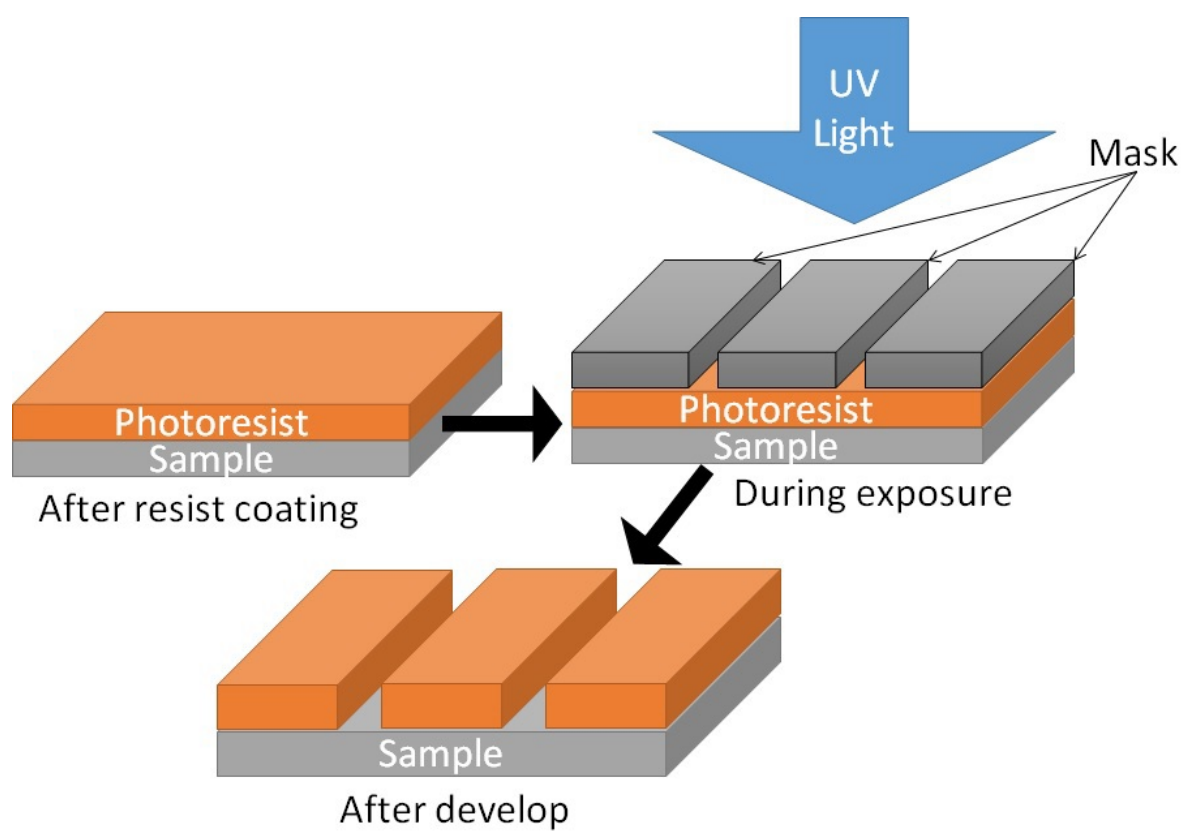

Figure F.1: A step by step process for imprinting pattern on sample surface with photolithography

First, the sample is cleand in ultrasonic bath of acetone, followed by isopropanol, and finally de-ionized water. This sample is baked in a hot plate at around $120^{\circ} \mathrm{C}$ to remove water from the surface. Laurel Technologies 400 Spinners is used for spin coating AZ5214 photo-resist. The photoresist is baked to cure. Suss Microtech MA6 Mask Aligner is used for alignment of the mask and exposure (320 nm UV light) of the photoresist in unmasked ares as shown in the figure F.1. Resists are made up of long chain of organic polymer which breaks down in UV light. The exposure time is carefully set so that the resist breaks down all the way to the sample surface. The exposed sample is developed in a specific developer which removes the exposed resist with the unexposed resist being intact. 


\section{F.2 Dry Etch}

Ion milling is used for etching away the parts of the sample in desired location at a controlled rate. In this method, high energy noble gas ion $\left(\mathrm{Ar}^{+}\right)$is directed to the sample surface by applying high voltage. These Argon ion bombard the sample surface in high vacuum environment and knock out the surface atoms. The process works by transferring the momentum of the $\mathrm{Ar}^{+}$to the atoms on the sample surface. Since Argon is an inert gas, using this method unwanted element doping to the sample is avoided. CVC 610 DC Magnetron Sputtering Station is used for dry etch as it is also equipped with an ion source.

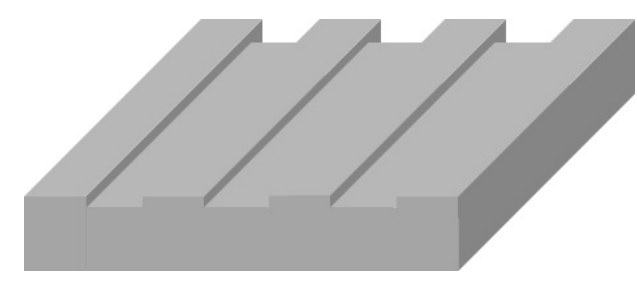

Figure F.2: The pattern imprinted on the sample surface after dry-etching the the exposed area after photolithography.

\section{F.3 Metal Deposition}

Metal deposition is done either by sputtering or e-beam evaporation. Figure F.3 shows the deposited metal after the lift off. During the deposition, metal are deposited everywhere. However, after the lift off, the metals remain only on the trenches created by photolithography. Metals deposited on the top of resist is removed during the lift off. 


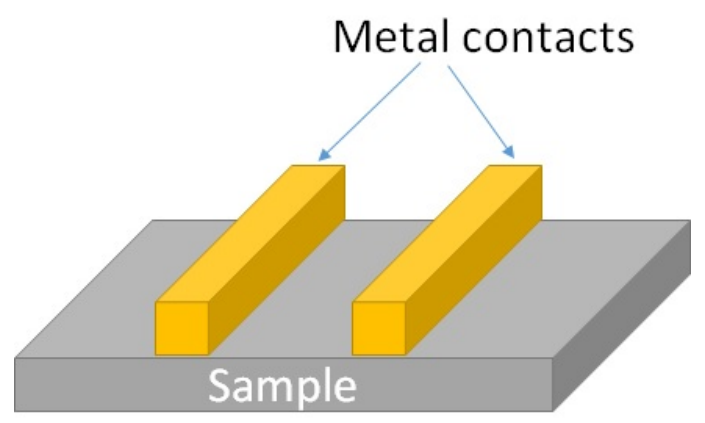

Figure F.3: A finished metal contacts deposited on sample surface after liftoff.

\section{F.3.1 Sputtering}

CVC 610 DC magnetron sputtering station is used for sputtering. In sputtering, a high voltage is used to breakdown Argon gas into Argon plasma. These plasma with very high energy bombarded on the sample surface to knock out the atoms of the target material. This atoms are than deposited on the sample which is placed just above the target.

\section{F.3.2 E-beam evaporation}

Temescal BJD 2000 e-beam evaporator is used for evaporation of the metal contacts. An electron-beam evaporator works by producing a high-energy (and high current) electron beam from an electron gun which is used to boil the target material of the choice. This evaporated target material gets deposited on the sample which is placed above it. 


\section{F.4 Oxygen Plasma Cleaning}

Oxygen plasma cleaning is a common cleanroom process for removing/modifying thin top surface in a sample as the plasma does not have enough energy to penetrate deep inside. Oxygen plasma is formed by breaking molecular oxygen into atomic oxygen than exciting the oxygen atoms and ionizing them with high frequency voltage. March PX-250 Plasma Asher was used for the plasma treatment of the sample. Plasma consists of many components of oxygen molecule such as: atomic oxygen, ionized ozone, metastably excited $O_{2}$, free electrons, etc. which can be represented by the following reactions [196]:

$$
\begin{gathered}
\mathrm{O}_{2}+e \rightarrow \mathrm{O}_{2}^{-} \rightarrow \mathrm{O}+\mathrm{O}^{-} \\
e+\mathrm{O} \rightarrow \mathrm{O}^{+}+2 e \\
e+\mathrm{O}_{2} \rightarrow \mathrm{O}_{2}^{+}+2 e \\
e+2 \mathrm{O}_{2} \rightarrow \mathrm{O}_{2}^{-}+\mathrm{O}_{2} \\
O_{2}^{-}+O \rightarrow O_{3}+e
\end{gathered}
$$

Where, e symbolizes an electron which has a negative charge. These reactions represent a small sampling of reactions that occur during the formation of oxygen plasma. These components can react with the top surface and modify it. 


\section{Appendix G}

\section{X-ray Photoelectron Spectroscopy}

Albert Einstein was awarded Nobel prize in physics in 1921 for photoelectric effect. X-ray Photoelectron Spectroscopy (XPS hereafter) is based on the same photoelectric effect. In XPS, a high voltage is applied to a metal to generate x-ray. This is then focused on the top of a sample which needs to be analyzed. These energetic x-rays have energy to access even the deep electron and eject them from their orbit. These ejected electron are collected and detected as shown in figure G.1. Since x-ray have wavelength comparable to the size of an atom, they can penetrate deep inside the material. However, the electron from deep inside cannot escape out

from the top surface. Hence, these electrons cannot be collected and studied. So, XPS can only analyze the surface, upto $5 \mathrm{~nm}$ deep inside [197]. 


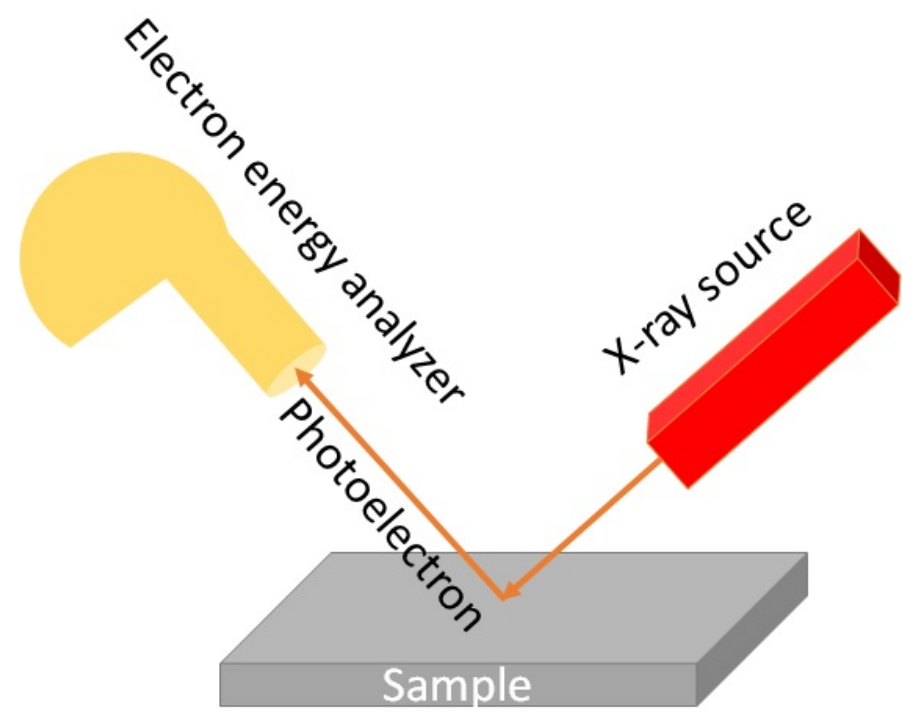

Figure G.1: A working mechanism of XPS.

An XPS spectra is a plot of binding energy vs. the number of electron detected. Mathematically, the binding energy is given by:

$$
E_{B E}=E_{p h}-\left(E_{k i}+\Phi\right)
$$

In equation above, $E_{B E}$ is the binding energy of the electron (unknown), $E_{p h}$ is the energy of the X-ray photons, $E_{k i}$ is the kinetic energy of the electron and $\phi$ is the work function (dependent on the spectrometer and the material). $E_{k i}$ is measured by the instrument and rest are known parameters. Hence, binding energy can be easily calculated. Each electron in an element have characteristic binding energy values that can directly identify the element. On the XPS plot each peak at different binding energy corresponds to the electron configuration of the electrons in that atom, e.g., $1 s, 2 s, 2 p, 3 s$, etc. An example XPS spectra from $\mathrm{Sm} 4 d$ in $\mathrm{SmB}_{6}$ is given in figure G.2. As mentioned earlier, the plot is counts vs. binding energy. 


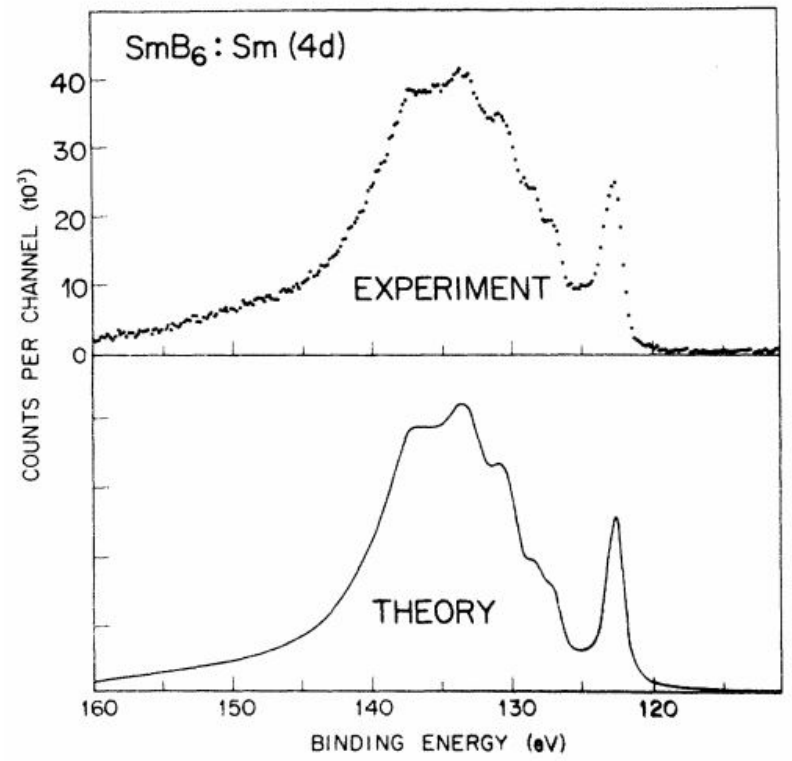

Figure G.2: XPS spectra of $\mathrm{Sm} 4 d$ spectrum in $\mathrm{SmB}_{6}$ [198]. The top data is from the experiment and the bottom is from theory. 


\section{Appendix $\mathbf{H}$}

\section{AFM}

AFM is an abbreviation for Atomic Force Microscopy. AFM is a type of scanning probe microscope (SEM). The concept of AFM was proposed by Gerd Bennig of IBM in 1986 [199]. In an AFM, a cantilever with very sharp tip is used to scan the sample surface. The resolution of the AFM depends on the diameter of the tip. Smaller the diameter of the tip, higher is the resolution. When the tip is brought in close contact to the sample surface, forces between tip and sample will cause deflection of the cantilever. Mathematically, this force $(F)$ is given by:

$$
F=C \Delta z
$$

where, $\mathrm{C}$ is the force constant of the cantilever and $\Delta z$ is the deflection of the cantilever. The possible forces responsible for this bending include mechanical contact force, van der Waals forces, capillary forces, chemical bonding, electrostatic forces, magnetic forces, Casimir forces, solvation forces, etc [200]. 


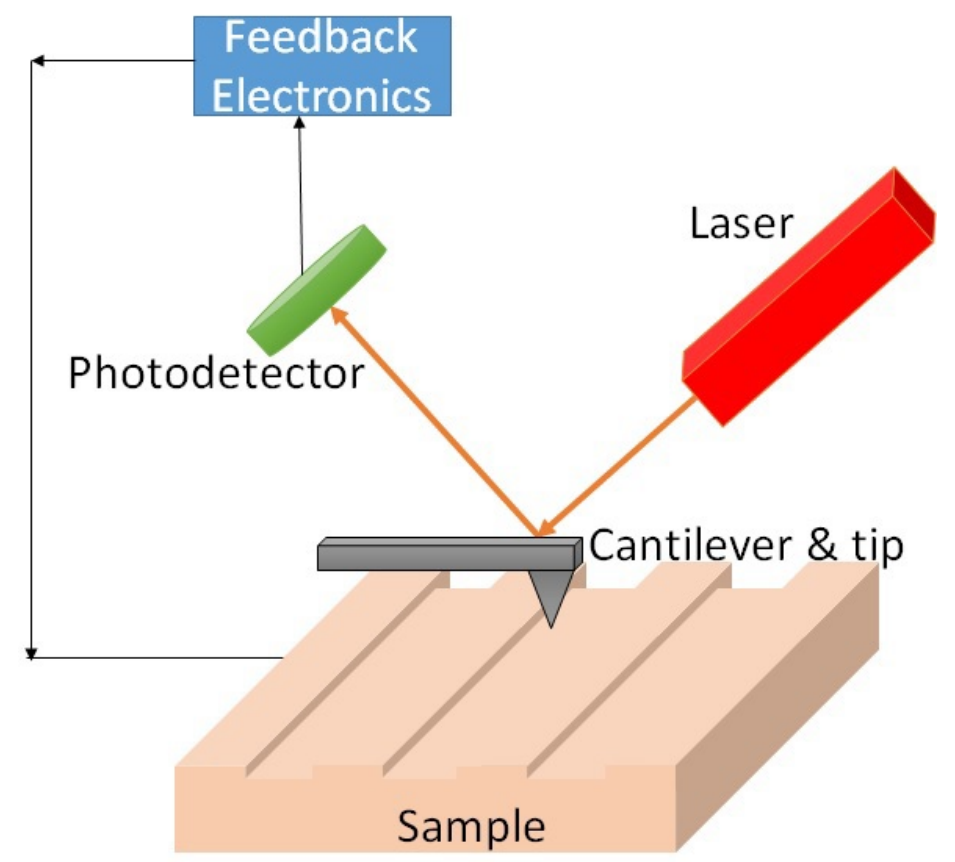

Figure H.1: A circuit diagram for AFM.

The deflection of the cantilever is measured using a laser reflected from the top surface of the cantilever (opposite to the tip) into a quadrent photodector (QPD)a as shown in figure H.1. The sample is mounted in a PZT (Pizeoelectric) scanner which can move in $\mathrm{x}, \mathrm{y}$ and $\mathrm{z}$ direction. The feedback mechanism is connected to the QPD and the sample stage which adjusts the tip-to-sample distance to maintain a constant force between the tip and the sample surface. Essentially, the cantilever is fixed in a position and the sample is moving in xyz direction which can spatially map out the top surface of the sample.

\section{H.1 AC mode}

In this mode, the tip does not come in contact with the sample surface. The cantilever is made to oscillate at the resonant frequency. During the scan, interac- 
tions between tip and sample surface will modulate the amplitude, phase, and the frequency of cantilever's vibrations. This change will be measured with a lock-in amplifier locked to the resonant frequency of the cantilever. Since there is no direct contact between sample and the tip, this mode is a gentle and used mostly for imaging topography.

\section{H.2 Contact Mode}

In contact mode, as name suggests, the tip is in contact with the sample surface all the time. As the tip scans the sample surface, the catiliver is bent depending on the surface topography. The laser reflected from the back of the cantilever is detected by a QPD. As the tip scans the surface, the reflected light hits different part of the QPD. The photovoltage generated by different detector in the QPD is used to read out of the interaction force between sample and tip.

\section{H.3 AFM Lithography}

In contact mode, voltage can be applied between the sample surface and a conductive probe. This will allow current to flow from the AFM tip to the sample surface. Since the size of the AFM tip is very small, a large electric field can be created between the sample surface and the AFM tip. This large field can alter the sample's electrical properties locally. Thus, a nanoscale lithography can be done on the sample surface using a voltage biased AFM tip in the contact mode. 


\section{Bibliography}

[1] A. Ohtomo and H. Hwang, "A high-mobility electron gas at the $\mathrm{LaAlO}_{3} / \mathrm{SrTiO}_{3}$ heterointerface," Nature, vol. 427, no. 6973, pp. 423-426, 2004.

[2] D. J. Kim, J. Xia, and Z. Fisk, "Topological surface state in the Kondo insulator samarium hexaboride," Nature Materials, vol. 13, pp. 466-470, May 2014.

[3] A. Menth, E. Buehler, and T. Geballe, "Magnetic and semiconducting properties of $\mathrm{SmB}_{6}, "$ Physical Review Letters, vol. 22, no. 7, p. 295, 1969.

[4] P. Schlottmann, "Impurity bands in kondo insulators," Physical Review B, vol. 46, no. 2, p. 998, 1992.

[5] J. Kondo, "Anomalous hall effect and magnetoresistance of ferromagnetic metals," Progress of Theoretical Physics, vol. 27, no. 4, pp. 772-792, 1962.

[6] M. Gmitra, H. Čenčariková, and P. Farkašovskỳ, "First-principles study of kondo insulator $\mathrm{SmB}_{6}$, , Acta Physica Polonica A, vol. 126, no. 1, pp. 298-299, 2014.

[7] G. Travaglini and P. Wachter, "Intermediate-valent $\mathrm{SmB}_{6}$ and the hybridization model: An optical study," Physical Review B, vol. 29, pp. 893-898, Jan. 1984.

[8] M. Dzero, K. Sun, V. Galitski, and P. Coleman, "Topological kondo insulators," Physical Review Letters, vol. 104, no. 10, p. 106408, 2010. PRL.

[9] P. Syers, D. Kim, M. S. Fuhrer, and J. Paglione, "Tuning bulk and surface conduction in the proposed topological kondo insulator $\mathrm{SmB}_{6}$," Physical review letters, vol. 114, no. 9, p. 096601, 2015.

[10] S. Wolgast, Ç. Kurdak, K. Sun, J. Allen, D.-J. Kim, and Z. Fisk, "Lowtemperature surface conduction in the kondo insulator $\mathrm{SmB}_{6}$, , Physical Review $B$, vol. 88, no. 18, p. 180405, 2013.

[11] C. Kane and J. Moore, "Topological insulators," Physics World, vol. 24, no. 2, pp. 32-36, 2011. 
[12] M. Dzero, K. Sun, P. Coleman, and V. Galitski, "Theory of topological kondo insulators," Phys Rev B, vol. 85, no. 4, p. 045130, 2012.

[13] T. Takimoto, "Smb6: A promising candidate for a topological insulator," Journal of the Physical Society of Japan, vol. 80, no. 12, p. 123710, 2011.

[14] V. Alexandrov, M. Dzero, and P. Coleman, "Cubic topological kondo insulators," Phys Rev Lett, vol. 111, no. 22, p. 226403, 2013.

[15] F. Lu, J. Zhao, H. Weng, Z. Fang, and X. Dai, "Correlated topological insulators with mixed valence," Physical Review Letters, vol. 110, no. 9, p. 096401, 2013. PRL.

[16] Y. S. Kim, M. Brahlek, N. Bansal, E. Edrey, G. A. Kapilevich, K. Iida, M. Tanimura, Y. Horibe, S.-W. Cheong, and S. Oh, "Thickness-dependent bulk properties and weak antilocalization effect in topological insulator $\mathrm{Bi}_{2} \mathrm{Se}_{3}$," Phys Rev B, vol. 84, no. 7, p. 073109, 2011.

[17] J. Werner and F. F. Assaad, "Interaction-driven transition between topological states in a kondo insulator," Physical Review B, vol. 88, no. 3, p. 035113, 2013.

[18] C.-J. Kang, J. Kim, K. Kim, J. Kang, J. D. Denlinger, and B. I. Min, "Band symmetries of mixed-valence topological insulator: $\mathrm{SmB}_{6}$, Journal of the Physical Society of Japan, vol. 84, no. 2, p. 024722, 2015.

[19] R. Yu, H. Weng, X. Hu, Z. Fang, and X. Dai, "Model hamiltonian for topological kondo insulator $\mathrm{SmB}_{6}$," New Journal of Physics, vol. 17, no. 2, p. 023012, 2015.

[20] B. Roy, J. D. Sau, M. Dzero, and V. Galitski, "Surface theory of a family of topological kondo insulators," Physical Review B, vol. 90, no. 15, p. 155314, 2014.

[21] M. Legner, A. Rüegg, and M. Sigrist, "Topological invariants, surface states, and interaction-driven phase transitions in correlated kondo insulators with cubic symmetry," Physical Review B, vol. 89, no. 8, p. 085110, 2014.

[22] L. Fu and C. L. Kane, "Superconducting proximity effect and majorana fermions at the surface of a topological insulator," Physical review letters, vol. 100, no. 9, p. 096407, 2008.

[23] E. Majorana, "Theory of the symmetry of electrons and positrons," Nuovo Cimento, vol. 14, pp. 171-184, 1937.

[24] D. Kim, S. Thomas, T. Grant, J. Botimer, Z. Fisk, and J. Xia, "Surface hall effect and nonlocal transport in smb6: evidence for surface conduction," Scientific reports, vol. 3, 2013. 
[25] S. Thomas, d. J. Kim, S. B. Chung, T. Grant, Z. Fisk, and J. Xia, "Weak antilocalization and linear magnetoresistance in the surface state of $\mathrm{SmB}_{6}$," arXiv:130\%.4133, 2013.

[26] X. Zhang, N. P. Butch, P. Syers, S. Ziemak, R. L. Greene, and J. Paglione, "Hybridization, inter-ion correlation, and surface states in the kondo insulator SmB 6 ," Phys. Rev. X, vol. 3, no. 1, p. 011011, 2013.

[27] S. Rößler, T.-H. Jang, D.-J. Kim, L. H. Tjeng, Z. Fisk, F. Steglich, and S. Wirth, "Hybridization gap and fano resonance in $\mathrm{SmB}_{6}$," Proceedings of the National Academy of Sciences, vol. 111, no. 13, pp. 4798-4802, 2014.

[28] W. Ruan, C. Ye, M. Guo, F. Chen, X. Chen, G.-M. Zhang, and Y. Wang, "Emergence of a coherent in-gap state in the $\mathrm{SmB}_{6}$ kondo insulator revealed by scanning tunneling spectroscopy," Physical Review Letters, vol. 112, no. 13, p. 136401, 2014. PRL.

[29] N. Xu, X. Shi, P. K. Biswas, C. E. Matt, R. S. Dhaka, Y. Huang, N. C. Plumb, M. Radovic, J. H. Dil, and E. Pomjakushina, "Surface and bulk electronic structure of the strongly correlated system $\mathrm{SmB}_{6}$ and implications for a topological kondo insulator," Phys Rev B, vol. 88, no. 12, p. 121102, 2013.

[30] C.-H. Min, P. Lutz, S. Fiedler, B. Kang, B. Cho, H.-D. Kim, H. Bentmann, and F. Reinert, "Importance of charge fluctuations for the topological phase in $\mathrm{SmB}_{6}, "$ Physical review letters, vol. 112, no. 22, p. 226402, 2014.

[31] M. Neupane, N. Alidoust, S. Y. Xu, T. Kondo, Y. Ishida, D. J. Kim, C. Liu, I. Belopolski, Y. J. Jo, T. R. Chang, H. T. Jeng, T. Durakiewicz, L. Balicas, H. Lin, A. Bansil, S. Shin, Z. Fisk, and M. Z. Hasan, "Surface electronic structure of the topological kondo-insulator candidate correlated electron system $\mathrm{SmB}_{6}, "$ Nat Commun, vol. 4, 2013.

[32] J. Denlinger, J. Allen, J.-S. Kang, K. Sun, J.-W. Kim, J. Shim, B. Min, D.-J. Kim, and Z. Fisk, "Temperature dependence of linked gap and surface state evolution in the mixed valent topological insulator $\mathrm{SmB}_{6}$," arXiv preprint arXiv:1312.6637, 2013.

[33] G. Li, Z. Xiang, F. Yu, T. Asaba, B. Lawson, P. Cai, C. Tinsman, A. Berkley, S. Wolgast, Y. S. Eo, D.-J. Kim, C. Kurdak, J. W. Allen, K. Sun, X. H. Chen, Y. Y. Wang, Z. Fisk, and L. Li, "Two-dimensional fermi surfaces in kondo insulator $\mathrm{SmB}_{6}$," Science, vol. 346, no. 6214, pp. 1208-1212, 2014.

[34] R. Monnier and B. Delley, "Properties of $\mathrm{LaB}_{6}$ elucidated by density functional theory," Physical Review B, vol. 70, no. 19, p. 193403, 2004. 
[35] M. Trenary, "Surface science studies of metal hexaborides," Science and Technology of Advanced Materials, vol. 13, no. 2, p. 023002, 2012.

[36] N. Heming, U. Treske, M. Knupfer, B. Büchner, D. Inosov, N. Shitsevalova, V. Filipov, S. Krause, and A. Koitzsch, "Surface properties of $\mathrm{SmB}_{6}$ from xray photoelectron spectroscopy," Physical Review B, vol. 90, no. 19, p. 195128, 2014.

[37] M. M. Yee, Y. He, A. Soumyanarayanan, D.-J. Kim, Z. Fisk, and J. E. Hoffman, "Imaging the kondo insulating gap on $\mathrm{SmB}_{6}$," arXiv preprint arXiv:1308.1085, 2013.

[38] Z.-H. Zhu, A. Nicolaou, G. Levy, N. Butch, P. Syers, X. Wang, J. Paglione, G. Sawatzky, I. Elfimov, and A. Damascelli, "Polarity-driven surface metallicity in $\mathrm{SmB}_{6}$," Physical review letters, vol. 111, no. 21, p. 216402, 2013.

[39] E. Frantzeskakis, N. de Jong, B. Zwartsenberg, Y. Huang, Y. Pan, X. Zhang, J. Zhang, F. Zhang, L. Bao, O. Tegus, et al., "Kondo hybridization and the origin of metallic states at the (001) surface of $\mathrm{SmB}_{6}, "$ Physical Review $X$, vol. 3, no. 4, p. 041024, 2013.

[40] I. Batko and M. Batkova, "SmB 6 : Topological insulator or semiconductor with valence-fluctuation induced hopping transport?," Solid State Communications, vol. 196, pp. 18-23, 2014.

[41] C. W. Luo, I. H. Wu, P. C. Cheng, J. Y. Lin, K. H. Wu, T. M. Uen, J. Y. Juang, T. Kobayashi, D. A. Chareev, O. S. Volkova, and A. N. Vasiliev, "Quasiparticle dynamics and phonon softening in fese superconductors," Physical Review Letters, vol. 108, no. 25, p. 257006, 2012. PRL.

[42] J. Demsar, V. K. Thorsmølle, J. L. Sarrao, and A. J. Taylor, "Photoexcited electron dynamics in kondo insulators and heavy fermions," Physical Review Letters, vol. 96, no. 3, p. 037401, 2006. PRL.

[43] K. H. Ahn, M. J. Graf, S. A. Trugman, J. Demsar, R. D. Averitt, J. L. Sarrao, and A. J. Taylor, "Ultrafast quasiparticle relaxation dynamics in normal metals and heavy-fermion materials," Physical Review B, vol. 69, no. 4, p. 045114, 2004. PRB.

[44] J. Demsar, R. D. Averitt, A. J. Taylor, V. V. Kabanov, W. N. Kang, H. J. Kim, E. M. Choi, and S. I. Lee, "Pair-breaking and superconducting state recovery dynamics in $\mathrm{MgB}_{2}$," Physical Review Letters, vol. 91, no. 26, p. 267002, 2003. PRL. 
[45] D. H. Torchinsky, F. Mahmood, A. T. Bollinger, I. Božović, and N. Gedik, "Fluctuating charge-density waves in a cuprate superconductor," Nat Mater, vol. 12 , no. 5, pp. 387-391, 2013.

[46] T. Mertelj, V. V. Kabanov, C. Gadermaier, N. D. Zhigadlo, S. Katrych, J. Karpinski, and D. Mihailovic, "Distinct pseudogap and quasiparticle relaxation dynamics in the superconducting state of nearly optimally doped $\mathrm{SmFeAsO}_{0.8} \mathrm{~F}_{0.2}$ single crystals," Physical Review Letters, vol. 102, no. 11, p. 117002, 2009. PRL.

[47] V. Pavlov, R. Pisarev, V. Gridnev, E. Zhukov, D. Yakovlev, and M. Bayer, "Ultrafast optical pumping of spin and orbital polarizations in the antiferromagnetic mott insulators $\mathrm{R}_{2} \mathrm{CuO}_{4}, "$ Physical review letters, vol. 98, no. 4, p. $047403,2007$.

[48] N. Laurita, C. Morris, S. Koopayeh, P. Cottingham, W. Phelan, L. Schoop, T. M. McQueen, and A. N. P, "Terahertz transmission studies of the topological Kondo insulator candidate $\mathrm{SmB}_{6}$," in Bulletin of the American Physical Society, vol. 59, American Physical Society, 20014.

[49] J. Zhang, J. Yong, I. Takeuchi, R. Greene, and R. Averitt, "Ultrafast quasiparticle dynamics of kondo insulator $\mathrm{SmB}_{6}$ using thz spectroscopy," in Bulletin of the American Physical Society, vol. 60, American Physical Society, 2015.

[50] C. Morris, N. Laurita, S. Koopayeh, P. Cottingham, W. Phelan, L. Schoop, T. McQueen, and N. Armitage, "Terahertz transmission and reflection studies of the topological kondo insulator candidate $\mathrm{SmB}_{6}$," in Bulletin of the American Physical Society, vol. 60, American Physical Society, 2015.

[51] Y. Ishida, T. Otsu, T. Shimada, M. Okawa, Y. Kobayashi, F. Iga, Y. Takabatake, and S. Shin, "Emergent photovoltage on $\mathrm{SmB}_{6}$ surface upon bulk-gap evolution revealed by pump-and-probe photoemission spectroscopy," Scientific reports, vol. 5, 2015.

[52] P. Hosur, "Circular photogalvanic effect on topological insulator surfaces: Berry-curvature-dependent response," Physical Review B, vol. 83, no. 3, p. 035309, 2011.

[53] J. McIver, D. Hsieh, H. Steinberg, P. Jarillo-Herrero, and N. Gedik, "Control over topological insulator photocurrents with light polarization," Nature nanotechnology, vol. 7, no. 2, pp. 96-100, 2012.

[54] D. Fu and M. Itoh, "Ferroelectricity in Silver Perovskite Oxides," in Ferroelectrics - Material Aspects (M. Lallart, ed.), InTech, aug 2011. 
[55] K. Yoshimatsu, R. Yasuhara, H. Kumigashira, and M. Oshima, "Origin of metallic states at the heterointerface between the band insulators $\mathrm{LaAlO}_{3}$ and $\mathrm{SrTiO}_{3}, "$ Physical review letters, vol. 101, no. 2, p. 026802, 2008.

[56] S. Thiel, G. Hammerl, A. Schmehl, C. Schneider, and J. Mannhart, "Tunable quasi-two-dimensional electron gases in oxide heterostructures," Science, vol. 313, no. 5795, pp. 1942-1945, 2006.

[57] A. Brinkman, M. Huijben, M. Van Zalk, J. Huijben, U. Zeitler, J. Maan, W. Van der Wiel, G. Rijnders, D. Blank, and H. Hilgenkamp, "Magnetic effects at the interface between non-magnetic oxides," Nature materials, vol. 6, no. 7, pp. 493-496, 2007.

[58] N. Reyren, S. Thiel, A. Caviglia, L. F. Kourkoutis, G. Hammerl, C. Richter, C. Schneider, T. Kopp, A.-S. Rüetschi, D. Jaccard, et al., "Superconducting interfaces between insulating oxides," Science, vol. 317, no. 5842, pp. 11961199, 2007.

[59] C. Cen, S. Thiel, J. Mannhart, and J. Levy, "Oxide nanoelectronics on demand," Science, vol. 323, no. 5917, pp. 1026-1030, 2009.

[60] G. Cheng, P. F. Siles, F. Bi, C. Cen, D. F. Bogorin, C. W. Bark, C. M. Folkman, J.-W. Park, C.-B. Eom, G. Medeiros-Ribeiro, et al., "Sketched oxide single-electron transistor," Nature Nanotechnology, vol. 6, no. 6, pp. 343-347, 2011.

[61] P. Irvin, Y. Ma, D. F. Bogorin, C. Cen, C. W. Bark, C. M. Folkman, C.-B. Eom, and J. Levy, "Rewritable nanoscale oxide photodetector," Nature Photonics, vol. 4, no. 12, pp. 849-852, 2010.

[62] Z. S. Popović, S. Satpathy, and R. M. Martin, "Origin of the two-dimensional electron gas carrier density at the $\mathrm{LaAlO}_{3}$ on $\mathrm{SrTiO}_{3}$ interface," Physical review letters, vol. 101, no. 25, p. 256801, 2008.

[63] J. Lee and A. A. Demkov, "Charge origin and localization at the n-type $\mathrm{SrTiO}_{3} / \mathrm{LaAlO}_{3}$ interface," Physical Review B, vol. 78, no. 19, p. 193104, 2008.

[64] N. Nakagawa, H. Y. Hwang, and D. A. Muller, "Why some interfaces cannot be sharp," Nature materials, vol. 5, no. 3, pp. 204-209, 2006.

[65] A. Kalabukhov, R. Gunnarsson, J. Börjesson, E. Olsson, T. Claeson, and D. Winkler, "Effect of oxygen vacancies in the $\mathrm{SrTiO}_{3}$ substrate on the electrical properties of the $\mathrm{LaAlO}_{3} / \mathrm{SrTiO}_{3}$ interface," Physical Review B, vol. 75, no. 12, p. 121404, 2007. 
[66] J. Mannhart and D. Schlom, "Oxide interfaces-an opportunity for electronics," Science, vol. 327, no. 5973, pp. 1607-1611, 2010.

[67] A. Janotti, L. Bjaalie, L. Gordon, and C. Van de Walle, "Controlling the density of the two-dimensional electron gas at the $\mathrm{SrTiO}_{3} / \mathrm{LaAlO}_{3}$ interface," Physical Review B, vol. 86, no. 24, p. 241108, 2012.

[68] E. Lesne, N. Reyren, D. Doennig, R. Mattana, H. Jaffrès, V. Cros, F. Petroff, F. Choueikani, P. Ohresser, R. Pentcheva, et al., "Suppression of the critical thickness threshold for conductivity at the $\mathrm{LaAlO}_{3} / \mathrm{SrTiO}_{3}$ interface," Nature communications, vol. 5, 2014.

[69] R. Pentcheva, R. Arras, K. Otte, V. G. Ruiz, and W. E. Pickett, "Termination control of electronic phases in oxide thin films and interfaces: $\mathrm{LaAlO}_{3} / \mathrm{SrTiO}_{3}$ (001)," Philosophical Transactions of the Royal Society of London A: Mathematical, Physical and Engineering Sciences, vol. 370, no. 1977, pp. 4904-4926, 2012.

[70] Y. Shi, S. Wang, Y. Zhou, H. Ding, and D. Wu, "Tuning the carrier density of $\mathrm{LaAlO}_{3} / \mathrm{SrTiO}_{3}$ interfaces by capping la1- xsrxmno3," Applied Physics Letters, vol. 102, no. 7, p. $071605,2013$.

[71] K. Au, D. Li, N. Chan, and J. Dai, "Polar liquid molecule induced transport property modulation at $\mathrm{LaAlO}_{3} / \mathrm{SrTiO}_{3}$ heterointerface," Advanced Materials, vol. 24, no. 19, pp. 2598-2602, 2012.

[72] L. Yu and A. Zunger, "A polarity-induced defect mechanism for conductivity and magnetism at polar-nonpolar oxide interfaces," Nature communications, vol. 5, 2014.

[73] C. Cen, S. Thiel, G. Hammerl, C. Schneider, K. Andersen, C. Hellberg, J. Mannhart, and J. Levy, "Nanoscale control of an interfacial metal-insulator transition at room temperature," Nature materials, vol. 7, no. 4, pp. 298-302, 2008.

[74] F. Bi, D. F. Bogorin, C. Cen, C. W. Bark, J.-W. Park, C.-B. Eom, and J. Levy, "Erratum: 'Water-cycle' mechanism for writing and erasing nanostructures at the $\mathrm{LaAlO}_{3} / \mathrm{SrTiO}_{3}$ interface" [appl. phys. lett. 97, 173110 (2010)]," Applied Physics Letters, vol. 100, no. 19, pp.--, 2012.

[75] J. C. Ho, R. Yerushalmi, Z. A. Jacobson, Z. Fan, R. L. Alley, and A. Javey, "Controlled nanoscale doping of semiconductors via molecular monolayers," Nature Materials, vol. 7, no. 1, pp. 62-67, 2008. 
[76] P. Zhang, E. Tevaarwerk, B.-N. Park, D. E. Savage, G. K. Celler, I. Knezevic, P. G. Evans, M. A. Eriksson, and M. G. Lagally, "Electronic transport in nanometre-scale silicon-on-insulator membranes," Nature, vol. 439, no. 7077, pp. 703-706, 2006.

[77] D. Watanabe, A. En, S. Nakamura, M. Suhara, and T. Okumura, "Anomalously large band-bending for hf-treated p-si surfaces," Applied surface science, vol. 216, no. 1, pp. 24-29, 2003.

[78] C. Di Valentin, G. Pacchioni, and A. Selloni, "Electronic structure of defect states in hydroxylated and reduced rutile $\mathrm{TiO}_{2}$ (110) surfaces," Physical review letters, vol. 97, no. 16, p. 166803, 2006.

[79] M. Jones, S. S. Lo, and G. D. Scholes, "Quantitative modeling of the role of surface traps in cdse/cds/zns nanocrystal photoluminescence decay dynamics," Proceedings of the National Academy of Sciences, vol. 106, no. 9, pp. 3011-3016, 2009.

[80] P. Strobel, M. Riedel, J. Ristein, and L. Ley, "Surface transfer doping of diamond," Nature, vol. 430, no. 6998, pp. 439-441, 2004.

[81] V. Chakrapani, J. C. Angus, A. B. Anderson, S. D. Wolter, B. R. Stoner, and G. U. Sumanasekera, "Charge transfer equilibria between diamond and an aqueous oxygen electrochemical redox couple," Science, vol. 318, no. 5855, pp. 1424-1430, 2007.

[82] P. L. Levesque, S. S. Sabri, C. M. Aguirre, J. Guillemette, M. Siaj, P. Desjardins, T. Szkopek, and R. Martel, "Probing charge transfer at surfaces using graphene transistors," Nano letters, vol. 11, no. 1, pp. 132-137, 2010.

[83] C. Coletti, C. Riedl, D. S. Lee, B. Krauss, L. Patthey, K. von Klitzing, J. H. Smet, and U. Starke, "Charge neutrality and band-gap tuning of epitaxial graphene on sic by molecular doping," Physical Review B, vol. 81, no. 23, p. 235401, 2010.

[84] R. He and P. Yang, "Giant piezoresistance effect in silicon nanowires," Nature nanotechnology, vol. 1, no. 1, pp. 42-46, 2006.

[85] S. Kobayashi, T. Nishikawa, T. Takenobu, S. Mori, T. Shimoda, T. Mitani, H. Shimotani, N. Yoshimoto, S. Ogawa, and Y. Iwasa, "Control of carrier density by self-assembled monolayers in organic field-effect transistors," Nature materials, vol. 3, no. 5, pp. 317-322, 2004.

[86] A. L. Schawlow and C. H. Townes, "Infrared and Optical Masers," Physical Review, vol. 112, pp. 1940-1949, Dec. 1958. 
[87] N. Bohr, "On the constitution of atoms and molecules," The London, Edinburgh, and Dublin Philosophical Magazine and Journal of Science, vol. 26, no. 151, pp. 1-25, 1913.

[88] D. Griffiths, Introduction to Quantum Mechanics: Pearson New International Edition. Always learning, Pearson Education Limited, 2013.

[89] T. H. Maiman, "Stimulated optical radiation in ruby," Nature, 1960.

[90] P. F. Moulton, "Spectroscopic and laser characteristics of Ti: $\mathrm{Al}_{2} \mathrm{O}_{3}$," Journal of the Optical Society of America B, vol. 3, p. 125, Jan. 1986.

[91] K. Wall and A. Sanchez, "Titanium Sapphire Lasers," The Lincoln Laboratory Journal, vol. 3, no. 3, 1990.

[92] U. Morgner, F. X. Kärtner, S. H. Cho, Y. Chen, H. A. Haus, J. G. Fujimoto, E. P. Ippen, V. Scheuer, G. Angelow, and T. Tschudi, "Sub-two-cycle pulses from a kerr-lens mode-locked Ti:sapphire laser," Optics Letters, vol. 24, p. 411, Mar. 1999.

[93] D. W. Piston, J. C. Long, and M. Davidson, "Ti:sapphire mode-locked lasers," 2012.

[94] P. Milonni and J. Eberly, Lasers Physics. Wiley Series in Pure and Applied Optics, Wiley, 1988.

[95] U. Keller, "Recent developments in compact ultrafast lasers," Nature, vol. 424, no. 6950, pp. 831-838, 2003.

[96] M. T. Asaki, C.-P. Huang, D. Garvey, J. Zhou, H. C. Kapteyn, and M. M. Murnane, "Generation of 11-fs pulses from a self-mode-locked ti:sapphire laser," Opt. Lett., vol. 18, pp. 977-979, Jun 1993.

[97] M. Polyanskiy, "Refractive index database," 2015.

[98] R. L. Fork, O. E. Martinez, and J. P. Gordon, "Negative dispersion using pairs of prisms," Opt. Lett., vol. 9, pp. 150-152, May 1984.

[99] D. E. Spence, P. N. Kean, and W. Sibbett, "60-fsec pulse generation from a self-mode-locked ti: sapphire laser," Optics letters, vol. 16, no. 1, pp. 42-44, 1991.

[100] R. Szipocs, K. Ferencz, C. Spielmann, and F. Krausz, "Chirped multilayer coatings for broadband dispersion control in femtosecond lasers," Optics Letters, vol. 19, p. 201, Feb. 1994. 
[101] U. Morgner, F. X. Kärtner, S. H. Cho, Y. Chen, H. A. Haus, J. G. Fujimoto, E. P. Ippen, V. Scheuer, G. Angelow, and T. Tschudi, "Sub-two-cycle pulses from a kerr-lens mode-locked ti:sapphire laser," Opt. Lett., vol. 24, pp. 411-413, Mar 1999.

[102] P. A. Franken, A. E. Hill, C. W. Peters, and G. Weinreich, "Generation of Optical Harmonics," Physical Review Letters, vol. 7, no. 4, pp. 118-119, 1961.

[103] G. D. Boyd and D. A. Kleinman, "Parametric Interaction of Focused Gaussian Light Beams," Journal of Applied Physics, vol. 39, no. 8, pp. 3597-3639, 1968.

[104] J. Giordmaine and R. C. Miller, "Tunable coherent parametric oscillation in $\mathrm{LiNbO}_{3}$ at optical frequencies," Physical Review Letters, vol. 14, no. 24, p. 973, 1965.

[105] C. Fattinger and D. Grischkowsky, "Terahertz beams," Applied Physics Letters, vol. 54, pp. 490-492, feb 1989.

[106] A. Othonos, "Probing ultrafast carrier and phonon dynamics in semiconductors," Journal of Applied Physics, vol. 83, no. 4, 1998.

[107] A. Shavorskiy, A. Cordones, J. Vura-Weis, K. Siefermann, D. Slaughter, F. Sturm, F. Weise, H. Bluhm, M. Strader, H. Cho, M.-F. Lin, C. Bacellar, C. Khurmi, M. Hertlein, J. Guo, T. Tyliszczak, D. Prendergast, G. Coslovich, J. Robinson, R. A. Kaindl, R. W. Schoenlein, A. Belkacem, T. Weber, D. M. Neumark, S. R. Leone, D. Nordlund, H. Ogasawara, A. R. Nilsson, O. Krupin, J. J. Turner, W. F. Schlotter, M. R. Holmes, P. A. Heimann, M. Messerschmidt, M. P. Minitti, M. Beye, S. Gul, J. Z. Zhang, N. Huse, and O. Gessner, "Timeresolved x-ray photoelectron spectroscopy techniques for real-time studies of interfacial charge transfer dynamics," in AIP Conference Proceedings, vol. 1525, AIP Publishing, 2013.

[108] D. Grischkowsky, S. Keiding, M. v. Exter, and C. Fattinger, "Far-infrared timedomain spectroscopy with terahertz beams of dielectrics and semiconductors," Journal of the Optical Society of America B, vol. 7, no. 10, p. 2006, 1990.

[109] J. Williamson, M. Dantus, S. Kim, and A. Zewail, "Ultrafast diffraction and molecular structure," Chemical physics letters, vol. 196, no. 6, pp. 529-534, 1992.

[110] N. V. Tkachenko, Optical spectroscopy: methods and instrumentations. Elsevier, 2006.

[111] M. Dantus and P. Gross, Ultrafast Spectroscopy. Wiley-VCH Verlag GmbH \& Co. KGaA, 2007. 
[112] A. J. Sabbah and D. M. Riffe, "Femtosecond pump-probe reflectivity study of silicon carrier dynamics," Physical Review B, vol. 66, no. 16, p. 165217, 2002. PRB.

[113] Z. Fisk, J. L. Sarrao, S. L. Cooper, P. Nyhus, G. S. Boebinger, A. Passner, and P. C. Canfield, "Kondo insulators," Physica B, vol. 223-224, pp. 409-412, 1996.

[114] P. A. Alekseev, A. S. Ivanov, V. N. Lazukov, I. P. Sadikov, and A. Severing, "Temperature effects in phonon dispersion of $\mathrm{SmB}_{6}$ intermediate valence semiconductor," Physica B: Condensed Matter, vol. 180-181, Part 1, no. 0, pp. 281-283, 1992.

[115] P. A. Alekseev, A. S. Ivanov, B. Dorner, H. Schober, K. A. Kikoin, A. S. Mishchenko, V. N. Lazukov, E. S. Konovalova, B. P. Yu, A. Y. Rumyantsev, and I. P. Sadikov, "Lattice dynamics of intermediate valence semiconductor $\mathrm{SmB}_{6}, "$ EPL (Europhysics Letters), vol. 10, no. 5, p. 457, 1989.

[116] P. Nyhus, S. L. Cooper, Z. Fisk, and J. Sarrao, "Light scattering from gap excitations and bound states in $\mathrm{SmB}_{6}$, " Physical Review B, vol. 52, no. 20, pp. R14308-R14311, 1995. PRB.

[117] A. Rothwarf and B. N. Taylor, "Measurement of recombination lifetimes in superconductors," Physical Review Letters, vol. 19, no. 1, pp. 27-30, 1967. PRL.

[118] V. V. Kabanov, J. Demsar, and D. Mihailovic, "Kinetics of a superconductor excited with a femtosecond optical pulse," Physical Review Letters, vol. 95, no. 14, p. 147002, 2005. PRL.

[119] D. Talbayev, K. S. Burch, E. E. M. Chia, S. A. Trugman, J. X. Zhu, E. D. Bauer, J. A. Kennison, J. N. Mitchell, J. D. Thompson, J. L. Sarrao, and A. J. Taylor, "Hybridization and superconducting gaps in the heavy-fermion superconductor pucoga ${ }_{5}$ probed via the dynamics of photoinduced quasiparticles," Physical Review Letters, vol. 104, no. 22, p. 227002, 2010. PRL.

[120] U. Bockelmann and G. Bastard, "Phonon scattering and energy relaxation in two-, one-, and zero-dimensional electron gases," Physical Review B, vol. 42, no. 14, pp. 8947-8951, 1990. PRB.

[121] J. Jiang, S. Li, T. Zhang, Z. Sun, F. Chen, Z. R. Ye, M. Xu, Q. Q. Ge, S. Y. Tan, X. H. Niu, M. Xia, B. P. Xie, Y. F. Li, X. H. Chen, H. H. Wen, and D. L. Feng, "Observation of possible topological in-gap surface states in the kondo insulator $\mathrm{SmB}_{6}$ by photoemission," Nat Commun, vol. 4, 2013. 
[122] K. Flachbart, K. Gloos, E. Konovalova, Y. Paderno, M. Reiffers, P. Samuely, and P. Švec, "Energy gap of intermediate-valent $\mathrm{SmB}_{6}$ studied by point-contact spectroscopy," Physical Review B, vol. 64, no. 8, p. 085104, 2001. PRB.

[123] B. Gorshunov, N. Sluchanko, A. Volkov, M. Dressel, G. Knebel, A. Loidl, and S. Kunii, "Low-energy electrodynamics of $\mathrm{SmB}_{6}$," Physical Review B, vol. 59, no. 3, pp. 1808-1814, 1999. PRB.

[124] J. C. Cooley, M. C. Aronson, Z. Fisk, and P. C. Canfield, "SmB ${ }_{6}$ : Kondo insulator or exotic metal?," Physical Review Letters, vol. 74, no. 9, pp. 1629 1632, 1995. PRL.

[125] S. Wolgast, a. Kurdak, K. Sun, J. W. Allen, D.-J. Kim, and Z. Fisk, "Lowtemperature surface conduction in the kondo insulator $\mathrm{SmB}_{6}$," Phys Rev B, vol. 88, no. 18, p. 180405, 2013.

[126] R. M. Martin and J. Allen, "Theory of mixed valence: Metals or small gap insulators," Journal of Applied Physics, vol. 50, no. B11, pp. 7561-7566, 1979.

[127] J. Allen, B. Batlogg, and P. Wachter, "Large low-temperature hall effect and resistivity in mixed-valent $\mathrm{SmB}_{6}$," Physical Review B, vol. 20, no. 12, p. 4807, 1979 .

[128] A. J. Sabbah and D. M. Riffe, "Measurement of silicon surface recombination velocity using ultrafast pump-probe reflectivity in the near infrared," Journal of Applied Physics, vol. 88, no. 11, pp. 6954-6956, 2000.

[129] C. Thomsen, H. T. Grahn, H. J. Maris, and J. Tauc, "Surface generation and detection of phonons by picosecond light pulses," Physical Review B, vol. 34, no. 6, pp. 4129-4138, 1986. PRB.

[130] C. Thomsen, J. Strait, Z. Vardeny, H. J. Maris, J. Tauc, and J. J. Hauser, "Coherent phonon generation and detection by picosecond light pulses," Physical Review Letters, vol. 53, no. 10, pp. 989-992, 1984. PRL.

[131] D. Mandrus, J. L. Sarrao, A. Lacerda, A. Migliori, J. D. Thompson, and Z. Fisk, "Low-temperature thermal expansion of $\mathrm{SmB}_{6}$ : Evidence for a single energy scale in the thermodynamics of kondo insulators," Physical Review B, vol. 49, no. 23, pp. 16809-16812, 1994. PRB.

[132] W. A. Phelan, S. M. Koohpayeh, P. Cottingham, J. W. Freeland, J. C. Leiner, C. L. Broholm, and T. M. McQueen, "Correlation between bulk thermodynamic measurements and the low-temperature-resistance plateau in $\mathrm{SmB}_{6}$," Physical Review X, vol. 4, no. 3, p. 031012, 2014. PRX. 
[133] S. Gabáni, E. Bauer, S. Berger, K. Flachbart, Y. Paderno, C. Paul, V. Pavlík, and N. Shitsevalova, "Pressure-induced fermi-liquid behavior in the kondo insulator $\mathrm{SmB}_{6}$ : Possible transition through a quantum critical point," Physical Review B, vol. 67, no. 17, p. 172406, 2003. PRB.

[134] J. Derr, G. Knebel, D. Braithwaite, B. Salce, J. Flouquet, K. Flachbart, S. Gabáni, and N. Shitsevalova, "From unconventional insulating behavior towards conventional magnetism in the intermediate-valence compound $\mathrm{SmB}_{6}$," Physical Review B, vol. 77, no. 19, p. 193107, 2008. PRB.

[135] Sandeep, M. P. Ghimire, D. P. Rai, P. K. Patra, A. K. Mohanty, and R. K. Thapa, "Study of bulk modulus, volume, energy, lattice parameters and magnetic moments in rare earth hexaborides using density functional theory," Journal of Physics: Conference Series, vol. 377, no. 1, p. 012084, 2012.

[136] J. Linder, T. Yokoyama, and A. Sudbø, "Anomalous finite size effects on surface states in the topological insulator $\mathrm{Bi}_{2} \mathrm{Se}_{3}$, " Physical review B, vol. 80, no. 20, p. 205401, 2009.

[137] M. Hasan and C. Kane, "Colloquium: Topological insulators," Rev Mod Phys, vol. 82, no. 4, pp. 3045-3067, 2010.

[138] Y. L. Chen, J. G. Analytis, J. H. Chu, Z. K. Liu, S. K. Mo, X.-L. Qi, H. J. Zhang, D. H. Lu, X. Dai, and Z. Fang, "Experimental realization of a threedimensional topological insulator, $\mathrm{Bi}_{2} \mathrm{Te}_{3}$, " Science, vol. 325, no. 5937, pp. 178 $181,2009$.

[139] N. Sluchanko, V. Glushkov, S. Demishev, A. Pronin, A. Volkov, M. Kondrin, A. Savchenko, and S. Kunii, "Low-temperature transport anisotropy and manybody effects in $\mathrm{SmB}_{6}$," Physical Review B, vol. 64, no. 15, p. 153103, 2001.

[140] C. Kastl, C. Karnetzky, H. Karl, and A. W. Holleitner, "Ultrafast helicity control of surface currents in topological insulators with near-unity fidelity," Nature communications, vol. 6, 2015.

[141] C. Jiang, V. Shalygin, V. Y. Panevin, S. N. Danilov, M. Glazov, R. Yakimova, S. Lara-Avila, S. Kubatkin, and S. Ganichev, "Helicity-dependent photocurrents in graphene layers excited by midinfrared radiation of a $\mathrm{CO}_{2}$ laser," Physical Review B, vol. 84, no. 12, p. 125429, 2011.

[142] H. Diehl, V. Shalygin, V. Bel'kov, C. Hoffmann, S. Danilov, T. Herrle, S. Tarasenko, D. Schuh, C. Gerl, W. Wegscheider, et al., "Spin photocurrents in (110)-grown quantum well structures," New Journal of Physics, vol. 9, no. 9, p. 349, 2007. 
[143] S. D. Ganichev and W. Prettl, "Spin photocurrents in quantum wells," Journal of Physics: Condensed Matter, vol. 15, no. 20, p. R935, 2003.

[144] K. Cho, C.-T. Liang, Y. Chen, Y. Tang, and B. Shen, "Spin-dependent photocurrent induced by rashba-type spin splitting in $\mathrm{Al}_{0.25} \mathrm{Ga}_{0.75} N / \mathrm{GaN}$ heterostructures," Physical Review B, vol. 75, no. 8, p. 085327, 2007.

[145] S. Ganichev, E. Ivchenko, S. Danilov, J. Eroms, W. Wegscheider, D. Weiss, and W. Prettl, "Conversion of spin into directed electric current in quantum wells," Physical review letters, vol. 86, no. 19, p. 4358, 2001.

[146] B. Wittmann, S. Danilov, V. Bel'kov, S. Tarasenko, E. Novik, H. Buhmann, C. Brüne, L. Molenkamp, Z. Kvon, N. Mikhailov, et al., "Circular photogalvanic effect in $\mathrm{HgTe} / \mathrm{CdHgTe}$ quantum well structures," Semiconductor Science and Technology, vol. 25, no. 9, p. 095005, 2010.

[147] M. Bahramy, P. King, A. De La Torre, J. Chang, M. Shi, L. Patthey, G. Balakrishnan, P. Hofmann, R. Arita, N. Nagaosa, et al., "Emergent quantum confinement at topological insulator surfaces," Nature communications, vol. 3, p. 1159, 2012.

[148] B. C. Park, T.-H. Kim, K. I. Sim, B. Kang, J. W. Kim, B. Cho, K.-H. Jeong, M.-H. Cho, and J. H. Kim, "Terahertz single conductance quantum and topological phase transitions in topological insulator $\mathrm{Bi}_{2} \mathrm{Se}_{3}$ ultrathin films," Nature communications, vol. 6, 2015.

[149] A. Pertsova and C. M. Canali, "Probing the wavefunction of the surface states in bi2se3 topological insulator: a realistic tight-binding approach," New Journal of Physics, vol. 16, no. 6, p. 063022, 2014.

[150] C.-X. Liu, H. Zhang, B. Yan, X.-L. Qi, T. Frauenheim, X. Dai, Z. Fang, and S.-C. Zhang, "Oscillatory crossover from two-dimensional to three-dimensional topological insulators," Physical review B, vol. 81, no. 4, p. 041307, 2010.

[151] H.-Z. Lu, W.-Y. Shan, W. Yao, Q. Niu, and S.-Q. Shen, "Massive dirac fermions and spin physics in an ultrathin film of topological insulator," Physical review $B$, vol. 81, no. 11, p. 115407, 2010.

[152] J. Mannhart, D. Blank, H. Hwang, A. Millis, and J.-M. Triscone, "Twodimensional electron gases at oxide interfaces," MRS bulletin, vol. 33, no. 11, pp. 1027-1034, 2008.

[153] A. Ohtomo and H. Hwang, "Corrigendum: A high-mobility electron gas at the $\mathrm{LaAlO}_{3} / \mathrm{SrTiO}_{3}$ heterointerface," Nature, vol. 441, no. 7089, pp. 120-120, 2006. 
[154] A. Caviglia, S. Gariglio, N. Reyren, D. Jaccard, T. Schneider, M. Gabay, S. Thiel, G. Hammerl, J. Mannhart, and J.-M. Triscone, "Electric field control of the $\mathrm{LaAlO}_{3} / \mathrm{SrTiO}_{3}$ interface ground state," Nature, vol. 456, no. 7222, pp. 624-627, 2008.

[155] S. Gariglio, N. Reyren, A. Caviglia, and J. Triscone, "Superconductivity at the $\mathrm{LaAlO}_{3} / \mathrm{SrTiO}_{3}$ interface," Journal of Physics: Condensed Matter, vol. 21, no. 16, p. 164213, 2009.

[156] G. Cheng, M. Tomczyk, S. Lu, J. P. Veazey, M. Huang, P. Irvin, S. Ryu, H. Lee, C.-B. Eom, C. S. Hellberg, et al., "Electron pairing without superconductivity," Nature, vol. 521, no. 7551, pp. 196-199, 2015.

[157] B. Kalisky, J. A. Bert, B. B. Klopfer, C. Bell, H. K. Sato, M. Hosoda, Y. Hikita, H. Y. Hwang, and K. A. Moler, "Critical thickness for ferromagnetism in $\mathrm{LaAlO}_{3} / \mathrm{SrTiO}_{3}$ heterostructures," Nature communications, vol. 3, p. 922, 2012.

[158] J. A. Bert, B. Kalisky, C. Bell, M. Kim, Y. Hikita, H. Y. Hwang, and K. A. Moler, "Direct imaging of the coexistence of ferromagnetism and superconductivity at the $\mathrm{LaAlO}_{3} / \mathrm{SrTiO}_{3}$ interface," Nature physics, vol. 7, no. 10, pp. 767-771, 2011.

[159] X. Wang, G. Baskaran, Z. Liu, J. Huijben, J. Yi, A. Annadi, A. R. Barman, A. Rusydi, S. Dhar, Y. Feng, et al., "Electronic phase separation at the $\mathrm{LaAlO}_{3} / \mathrm{SrTiO}_{3}$ interface," Nature communications, vol. 2, p. 188, 2011.

[160] F. Bi, M. Huang, S. Ryu, H. Lee, C.-W. Bark, C.-B. Eom, P. Irvin, and J. Levy, "Room-temperature electronically-controlled ferromagnetism at the $\mathrm{LaAlO}_{3} / \mathrm{SrTiO}_{3}$ interface," Nature communications, vol. 5, 2014.

[161] A. Tebano, E. Fabbri, D. Pergolesi, G. Balestrino, and E. Traversa, "Room-temperature giant persistent photoconductivity in $\mathrm{SrTiO}_{3} / \mathrm{LaAlO}_{3}$ heterostructures," Acs Nano, vol. 6, no. 2, pp. 1278-1283, 2012.

[162] A. Rastogi, J. Pulikkotil, S. Auluck, Z. Hossain, and R. Budhani, "Photoconducting state and its perturbation by electrostatic fields in oxide-based two-dimensional electron gas," Physical Review B, vol. 86, no. 7, p. 075127, 2012.

[163] Y. Ma, M. Huang, S. Ryu, C. W. Bark, C.-B. Eom, P. Irvin, and J. Levy, "Broadband terahertz generation and detection at $10 \mathrm{~nm}$ scale," Nano letters, vol. 13, no. 6, pp. 2884-2888, 2013. 
[164] L. R. Merte, G. Peng, R. Bechstein, F. Rieboldt, C. A. Farberow, L. C. Grabow, W. Kudernatsch, S. Wendt, E. Lægsgaard, M. Mavrikakis, et al., "Watermediated proton hopping on an iron oxide surface," Science, vol. 336, no. 6083, pp. 889-893, 2012.

[165] S. Wendt, J. Matthiesen, R. Schaub, E. K. Vestergaard, E. Lægsgaard, F. Besenbacher, and B. Hammer, "Formation and splitting of paired hydroxyl groups on reduced $\mathrm{tio}_{2}$ (110)," Physical review letters, vol. 96, no. 6, p. 066107, 2006.

[166] S.-C. Li, Z. Zhang, D. Sheppard, B. D. Kay, J. White, Y. Du, I. Lyubinetsky, G. Henkelman, and Z. Dohnálek, "Intrinsic diffusion of hydrogen on rutile $\mathrm{TiO}_{2}$ (110)," Journal of the American Chemical Society, vol. 130, no. 28, pp. 90809088, 2008.

[167] G. Cheng, J. P. Veazey, P. Irvin, C. Cen, D. F. Bogorin, F. Bi, M. Huang, S. Lu, C.-W. Bark, S. Ryu, et al., "Anomalous transport in sketched nanostructures at the $\mathrm{LaAlO}_{3} / \mathrm{SrTiO}_{3}$ interface," Physical Review X, vol. 3, no. 1, p. 011021, 2013.

[168] C. S. Hellberg, "Unpublised works,"

[169] G. Kresse and J. Furthmüller, "Efficient iterative schemes for ab initio totalenergy calculations using a plane-wave basis set," Physical Review B, vol. 54, no. 16, p. 11169, 1996.

[170] G. Kresse and D. Joubert, "From ultrasoft pseudopotentials to the projector augmented-wave method," Physical Review B, vol. 59, no. 3, p. 1758, 1999.

[171] P. E. Blöchl, "Projector augmented-wave method," Physical Review B, vol. 50, no. 24, p. 17953, 1994.

[172] J. P. Perdew, A. Ruzsinszky, G. I. Csonka, O. A. Vydrov, G. E. Scuseria, L. A. Constantin, X. Zhou, and K. Burke, "Restoring the density-gradient expansion for exchange in solids and surfaces," Physical Review Letters, vol. 100, no. 13, p. 136406, 2008.

[173] J. Neugebauer and M. Scheffler, "Adsorbate-substrate and adsorbate-adsorbate interactions of na and k adlayers on al (111)," Physical Review B, vol. 46, no. 24, p. 16067, 1992.

[174] G. Lefevre, M. Duc, P. Lepeut, R. Caplain, and M. Fédoroff, "Hydration of $\gamma$-alumina in water and its effects on surface reactivity," Langmuir, vol. 18, no. 20, pp. 7530-7537, 2002. 
[175] D. B. Asay and S. H. Kim, "Evolution of the adsorbed water layer structure on silicon oxide at room temperature," The Journal of Physical Chemistry B, vol. 109, no. 35, pp. 16760-16763, 2005.

[176] A. Weddemann, I. Ennen, A. Regtmeier, C. Albon, A. Wolff, K. Eckstädt, N. Mill, M. K.-H. Peter, J. Mattay, C. Plattner, et al., "Review and outlook: from single nanoparticles to self-assembled monolayers and granular gmr sensors," Beilstein journal of nanotechnology, vol. 1, no. 1, pp. 75-93, 2010.

[177] N. Bristowe, P. Ghosez, P. Littlewood, and E. Artacho, "The origin of twodimensional electron gases at oxide interfaces: insights from theory," Journal of Physics: Condensed Matter, vol. 26, no. 14, p. 143201, 2014.

[178] M. Gabay and J.-M. Triscone, "Oxide heterostructures: Hund rules with a twist," Nature Physics, vol. 9, no. 10, pp. 610-611, 2013.

[179] H. Peng, H. Xiang, S.-H. Wei, S.-S. Li, J.-B. Xia, and J. Li, "Origin and enhancement of hole-induced ferromagnetism in first-row d 0 semiconductors," Physical review letters, vol. 102, no. 1, p. 017201, 2009.

[180] A. Liechtenstein, V. Anisimov, and J. Zaanen, "Density-functional theory and strong interactions: Orbital ordering in mott-hubbard insulators," Physical Review B, vol. 52, no. 8, p. R5467, 1995.

[181] S. Nazir, C. Bernal, and K. Yang, "Modulated two-dimensional charge-carrier density in $\mathrm{LaAlO}_{3}$-layer-doped $\mathrm{LaAlO}_{3} / \mathrm{SrTiO}_{3}$ heterostructure," ACS applied materials $\mathcal{E}$ interfaces, vol. 7, no. 9, pp. 5305-5311, 2015.

[182] K. Park, W. Lin, and F. Paesani, "Fast and slow proton transfer in ice: The role of the quasi-liquid layer and hydrogen-bond network," The Journal of Physical Chemistry B, vol. 118, no. 28, pp. 8081-8089, 2014.

[183] T. Pinkerton, D. Scovell, A. Johnson, B. Xia, V. Medvedev, and E. Stuve, "Electric field effects in ionization of water-ice layers on platinum," Langmuir, vol. 15, no. 3, pp. 851-856, 1999.

[184] D. L. Scovell, T. D. Pinkerton, V. K. Medvedev, and E. M. Stuve, "Phase transitions in vapor-deposited water under the influence of high surface electric fields," Surface science, vol. 457, no. 3, pp. 365-376, 2000.

[185] M. E. Tuckerman, D. Marx, and M. Parrinello, "The nature and transport mechanism of hydrated hydroxide ions in aqueous solution," Nature, vol. 417, no. 6892, pp. 925-929, 2002. 
[186] D. Marx, M. E. Tuckerman, J. Hutter, and M. Parrinello, "The nature of the hydrated excess proton in water," Nature, vol. 397, no. 6720, pp. 601-604, 1999.

[187] J. Pérez-Lustres, F. Rodriguez-Prieto, M. Mosquera, T. Senyushkina, N. Ernsting, and S. Kovalenko, "Ultrafast proton transfer to solvent: molecularity and intermediates from solvation-and diffusion-controlled regimes," Journal of the American Chemical Society, vol. 129, no. 17, pp. 5408-5418, 2007.

[188] J. Park, D. Bogorin, C. Cen, D. Felker, Y. Zhang, C. Nelson, C. Bark, C. Folkman, X. Pan, M. Rzchowski, et al., "Creation of a two-dimensional electron gas at an oxide interface on silicon," Nature communications, vol. 1, p. 94, 2010.

[189] R. Trebino, Frequency-Resolved Optical Gating: The Measurement of Ultrashort Laser Pulses: The Measurement of Ultrashort Laser Pulses. Frequencyresolved Optical Gating: The Measurement of Ultrashort Laser Pulses, Springer US, 2000.

[190] R. W. Boyd, Nonlinear optics. Academic press, 2003.

[191] ID-INC, PEM-90 Photoelastic Modulators. Hinds Instruments.

[192] M. Meade, Lock-in Amplifiers: Principles and Applications. IEE electrical measurement series, P. Peregrinus, 1983.

[193] P. C. Hobbs, "Ultrasensitive laser measurements without tears," Applied optics, vol. 36, no. 4, pp. 903-920, 1997.

[194] L. Macey, Model 2007 and 2017 User's Manual. New Focus, Inc.

[195] W. Thurber, "Hall effect measurements," 2008.

[196] A. Pizzi and K. L. Mittal, Handbook of Adhesive Technology, Revised and Expanded. CRC Press, aug 2003.

[197] C. Kittel, Introduction to solid state physics. Wiley, 2005.

[198] J. Chazalviel, M. Campagna, G. Wertheim, and P. Schmidt, "Study of valence mixing in $\mathrm{SmB}_{6}$ by x-ray photoelectron spectroscopy," Physical Review $B$, vol. 14, no. 10, p. 4586, 1976.

[199] G. Binnig, C. F. Quate, and C. Gerber, "Atomic force microscope," Physical review letters, vol. 56, no. 9, p. 930, 1986.

[200] B. Cappella and G. Dietler, "Force-distance curves by atomic force microscopy," Surface science reports, vol. 34, no. 1, pp. 1-104, 1999. 